\author{
UNIVERSIDADE DE SÃO PAULO \\ INSTITUTO DE MATEMATICA E ESTATISTICA
}

\title{
APROXIMACAO E NUCLEOS \\ ORTONORMALIZANTES
}

Mariella Violeta Sotelo Campos
Dissertação apresentada para obtenção do Grau de Mestre em Matemática

São Paulo

$-1989-$ 


\section{AGRADECIMENTOS}

Ao Professor Pedro Nowossad pela sua orientação dedicada e apoio em todos os sentidos para a realização deste trabalho.

Aos professores e colegas do IME.

000 
CAPITULO I - APROXIMAÇO POLINOMIAL E RACIONAL NO DOMINIO COMPLEXO

1. Teorema de Aproximação de Runge ............... 04

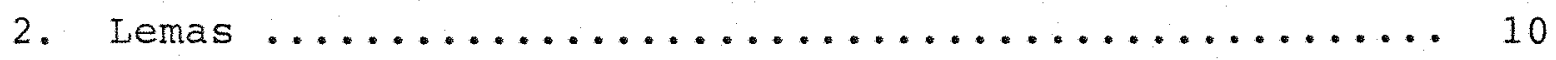

3. o Teorema de Mergelyan ...................... 16

4. Extensão do Teorema de Mergelyan para Dominios Multiplamente conexos.

CAPITULO II - A INTEGRAL DE CAUCHY - FÓRMULAS DE PLEMELJ

1. Definição da Integral de Cauchy em uma curva . ..... 30

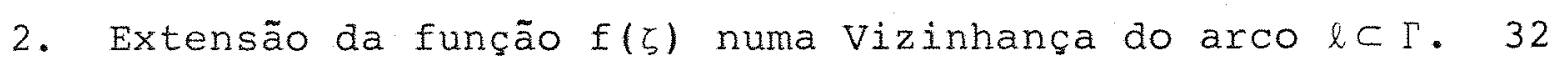

3. Comportamento da Integral de Cauchy nas Proximidades

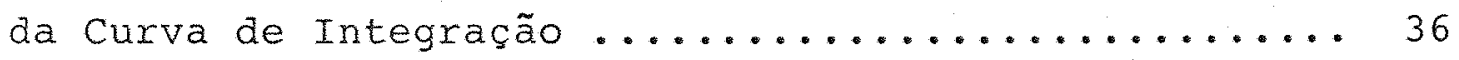

4. Propriedades de Fronteira da Integral de Cauchy.

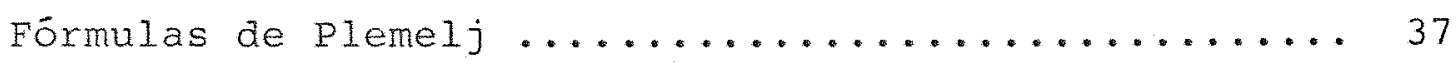

CAPITULO III - NOCLEOS ORTONORMALIZANTES PARA CURVAS FECHADAS SIMPLES

1. Definição e Forma explicita do Produto Interno ..... 40

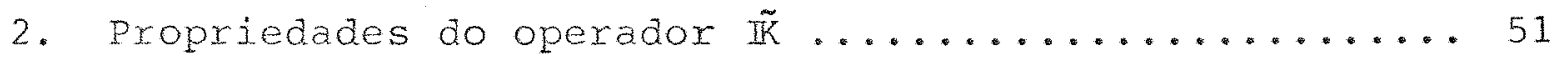

3. Uma Base Ortonormal para o Espaço $H_{r}(\Gamma,<,>) \ldots . . . .53$ 
4. Outra Base Ortonormal para o Espaco $\mathrm{H}_{\Gamma}(\Gamma,<,>) \ldots . . .57$

5. Caracterizações do Completamento de $\mathrm{H}_{Y}(\Gamma,<,>) \ldots . . .64$

6. Construção de um Espaço de Hilbert de Funções

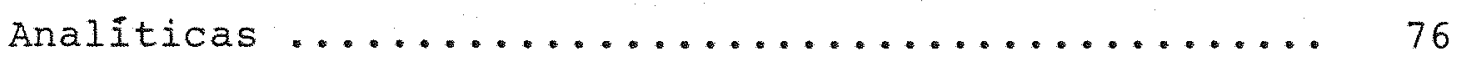

CAPITULO IV - APLICAÇ̃O A FORMA DIFERENCIAL $\bar{z} d z \ldots . . . .86$

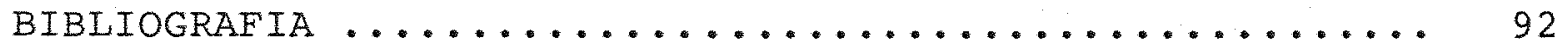

○O 


\section{INTRODUÇÃO}

Seja $s^{1}$ o círculo unitário no plano complexo. Sabe mos que o conjunto $\left\{e^{i n t}\right\}_{n \in \mathbb{Z}}$ é um sistema ortonormal comple xo em $L^{2}\left(S^{1}\right)$ em relação ao produto interno usual.

Se $f \in L^{2}\left(S^{1}\right)$, definimos seu n-ësimo coeficiente de Fourier

$$
\left(e^{i n t}, f\right)=\frac{1}{2 \pi} \int_{-\pi}^{\pi} f(t) e^{-i n t} d t, n \in \mathbb{Z}
$$

e a sërie de Fourier de $f$ è dada por

$$
\sum_{n=-\infty}^{+\infty}\left(e^{i n t}, f\right) e^{i n t},
$$

a qual converge a $f$ no sentido de $\mathrm{L}^{2}$.

Neste trabalho, definiremos um produto interno numa curva $\Gamma$ fechada e simples no plano, de modo que as potēncias $z^{n}$, n inteiro relativo, formem uma base ortogonal em relação a este produto interno. Este resultado generaliza num determinado sentido o conceito de séries de Fourier associado ao circulo unitário acima citado.

Veremos que, dada uma curva $T$ e escolhidas a origem no seu interior $\Omega$ e o disco $D(0 ; r) \subset \Omega, 0<r<1$, o espaco $\mathrm{H}_{r}(\Gamma,<,>)$, das funções Hölder continuas munido deste produto interno que definiremos possui a base o.n. $\left\{\theta_{m}=\frac{z^{m}}{\sqrt{2 \pi r}|m+1|}\right\}_{m \in Z Z}$ 
Dada uma função $f \in C(\Gamma)$ definiremos sua série formal de Laurent $\sum_{n=-\infty}^{+\infty} a_{n} z^{n}$ usando o Teorema de Mergelyan do Cá pitulo I, para dominios multiplamente conexos. Esta série,pu ramente formal em $C(\Gamma)$, convergirá a f no sentido de $H_{r}(\Gamma,\langle,>)$. Dai segue a seguinte propriedade dos coeficientes de Laurent:

$$
\sum_{n=-\infty}^{+\infty} r^{2|n+1|}\left|a_{n}\right|^{2}<+\infty
$$

Mostraremos que quando $\Gamma=S^{1}$ e $r \rightarrow 1$, nosso espaco $H_{I}\left(S^{1},<,>\right)$ coincide com o espaço $L^{2}\left(S^{1}\right)$.

Também, construimos um espaço de Hilbert $\mathscr{H}$ de funçōes analiticas em $\mathbb{C} \backslash \Gamma$, associado aos espaços $\mathrm{H}_{r}(\Gamma,\langle,>)$.

Finalmente, no Capitulo IV veremos uma conseqüência não trivial para o estudo da forma diferencial $\bar{\zeta} d \zeta$, dependendo da geometria da curva $\Gamma$. 


\section{CAPITULO I}

\section{APROXIMACAO POLINOMIAL E RACIONAL NO DOMINIO COMPLEXO}

Seja $K \in \mathbb{C}$ um conjunto compacto e $K^{\circ}=\operatorname{int}(K)$. Denotemos por

$P(K)=\left\{f: K+\mathbb{C} ; \mathbb{E}\right.$ sequência $\left\{P_{n}\right\}$ de polinômios com $\left.P_{n}(z) \frac{\text { unif }}{K} f(z)\right\}$ o germe de $\mathrm{K}$, e

$$
\begin{aligned}
R(K)=\left\{f: K \rightarrow \mathbb{C} ; \text { I sequência }\left\{R_{n}\right\}\right. \text { de funções racionais com } \\
\text { polos fora de } \left.K \text { e } \frac{R}{n} \frac{\text { unif }}{K} f\right\} .
\end{aligned}
$$

Seja f uma função contínua em $K$ e holomorfa em $K^{\circ}$. Neste primeiro capítulo estudaremos o seguinte problema da teoria de aproximação qualitativa: Sob que condições a função $f$ pode ser aproximada por polinômios ou funções racionais em $K$ ? Se $K=[a, b] \subset \mathbb{R}$, o Teorema de Aproximação de Weierstrass afirma:

$$
P(K)=C(K) \text {. }
$$

Por outro lado, o Teorema de Runge afirma que toda função $f \in C(K)$ que tenha extensão holomorfa a algun conjunto aberto $\Omega \supset K$ perten ce a $R(K)$ desde que tomemos como conjunto dos polos um. conjunto con pontos em cada componente conexa de $\mathrm{K}^{\mathrm{C}}$. No caso especjal, en que $\mathrm{K}^{\mathrm{C}}$ é conexo, todos os po los podem se mover para $\omega$ : neste caso, toda funçäo holomorfa en KCn pertence a $P(K)$. 
Finalmente, o Teorema de Mergelyen afirma que, se 0 compacto $K$ não separa o plano (i.e. $K^{c}$ é conexo) e fé contínua en $K$ e holomorfa em $K^{O}$ então $f \in P(K)$. Caso $K^{C}$ possua un nünero finito de componentes, temos $f \in R(K)$.

1. Teorema de Aproximação de Runge.

Teorema I.I.I - Seja $K \subset \mathbb{C}$ um conjunto compacto $e\left[a_{j}\right\}$ um conjunto que contém um ponto de cada componente de $S^{2} \backslash K$. Seja $\Omega \subset \mathbb{C}$ aberto tal que $K \subset \Omega, f \in A(\Omega)$, e seja $\in>0$ então existe uma função racional $R$, tel que todos os seus polos estão contidos no conjunto $\left\{\alpha_{j}\right\}$ e

$(1.1 .1)$

$$
|f(z)-R(z)|<\varepsilon \quad \forall z \in K
$$

Observacão: $S^{2} \backslash \mathrm{K}$ possui no máximo um conjunto enumerável de componentes.

Prova - Consideremos o espaço de Banach $\left(\mathrm{C}(\mathrm{K}),\|\cdot\|_{\infty}\right)$ onde $C(K)=\{f: K \rightarrow \mathbb{C} ; f$ continua $\}$ e $\|\cdot\|_{\infty}$ é a norma do supremo. Temos que $R(K)$ é subespaço fechado de $C(K)$. Pelo Teorema da Representação de Riesz, Ver 6 , pag. $1 \$ 1)$ todo funcional linear contínuo $\&$ sobre $C(\mathrm{~K})$ é dado por una única medida complexa boreliana $\mu$ sobre $K$ tal que

$$
\mathscr{L}(f)=\int_{K} f d \mu \quad \forall f \in C(K) .
$$

Assim, pelo Teorema de Separacão de Hahn-Banach (Ver 6 , pag. 108, teo rema 5.19), a funçäo $f$ em $C(K)$ pertence ao subespaço fechado $R(K)$ se e somente se para todas as medidas $w$ sobre $K$ que sejam ortogonais a $R(K)$, sejam também ortogonais para $f: \int_{K} f d u=0$.

Portanto, o teorema estará provado se demonstrarmos a 
seguinte

Afirmacão: Se $\mu$ é uma medida complexa de Borel sobre $K$ tal que

$$
\int_{K} R d \mu=0
$$

para toda função racional $R$ com polos somente no conjunto $\left\{a_{j}\right\}$, então para todo $f \quad A(\Omega)$ vale

$$
\int_{K} f d \mu=0
$$

Prova - Assuma que existe 4 medida complexa de Borel satisfazendo $(I, 1,2)$. Defina

$$
h(z)=\int_{K} \frac{d \mu(\zeta)}{\zeta-z}, \quad z \in S^{2} \backslash K
$$

então $h \in H\left(S^{2} \backslash K\right)$, De fato considerando o disco aberto de centro a e raio $r D(a ; r) \quad S^{2} / K$, com a $s^{2} / K$ e $r>0$, desde que $\zeta \quad K$, temos $\left|\zeta^{-a}\right|>r$ e (I.1.4 $) \quad\left|\frac{z-a}{z-\zeta}\right|<\frac{|z-a|}{r}<I \quad \forall z \in D(a ; r) e \quad \forall b \in K$. Portanto, a série geométrica

$$
\sum_{n=0}^{+\infty} \frac{(z-a)^{r}}{(\zeta-a)^{n+1}}=\frac{1}{\zeta-z}
$$

converge uniformemente sobre $K$, para todo $z \in D(a ; r)$. Daí substituindo a série (I.1.4') em (I.1.4) e integrando termo a termo obtemos

$$
h(z)=\sum_{n=0}^{+\infty}\left(\int_{K} \frac{d \mu(\zeta)}{(\zeta-a)^{n+I}}\right)(z-a)^{n} \text { para todo } z \in D(a ; r)
$$


Então f possui uma representação em séries de potências em $S^{2} \backslash K, \log 0 f \in A\left(S^{2} \backslash K\right)$. Agora, seja $V_{j}$ uma componente de $S^{2} \backslash K$ tal que $a_{j} \in V_{j}$ e suponha que $D\left(a_{j} ; r\right) \subset V_{j}, r>0$

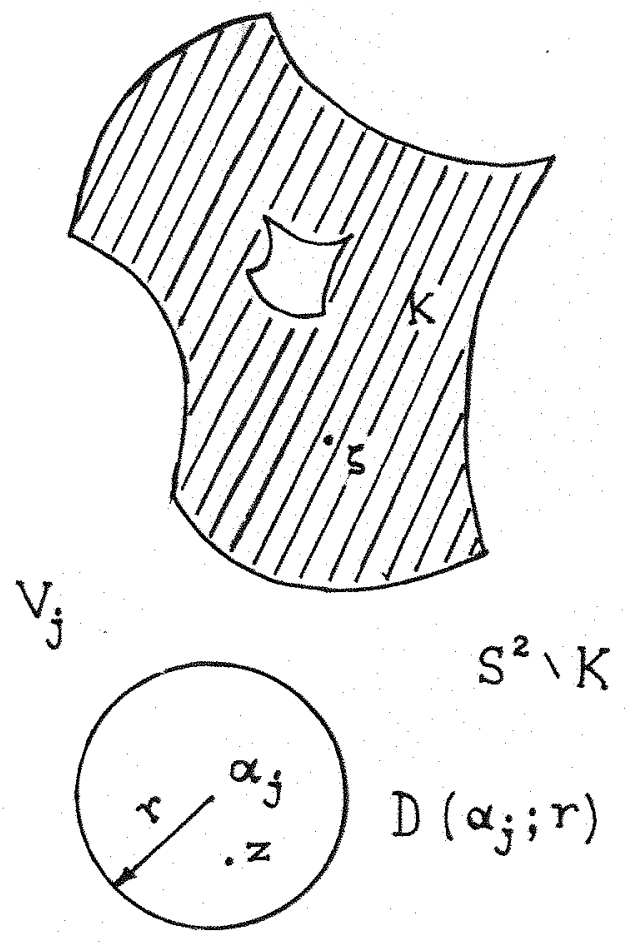

Vamos considerar dois casos:

a) Se $a_{j} \neq \infty$, seja $z \in D\left(a_{j} ; r\right)$ fixo, temos

$$
\frac{1}{\xi-2}=\frac{1}{\xi-\alpha_{j}} \cdot \frac{1}{1-\frac{z-\alpha_{j}}{\xi-\alpha_{j}}}
$$

Já que

$$
\left|\frac{z-a_{j}}{g-a_{j}}\right|<I \quad \forall \xi \in K
$$

então

$$
\frac{1}{\xi-z}=\lim _{N \rightarrow+\infty} \sum_{n=0}^{N} \frac{\left(z-\alpha_{j}\right)^{n}}{\left(\xi-\alpha_{j}\right)^{n+1}}
$$

converge uniformemente $\forall \xi \in K$. 
Como cada uma das funções $\sum_{n=0}^{N} \frac{\left(z-a_{j}\right)^{n}}{\left(\zeta-a_{j}\right)^{n+1}}$ é uma função racional, então pode-se aplicar (I.1.2), daí:

$$
h(z)=0 \quad \text { para todo } z \in D\left(a_{j} ; r\right)
$$

Logo $h(z)=0$ para todo $z \in V_{j}$.

b) Se $a_{j}=\infty$, seja $\zeta \in K$ e $|z|>r, z$ fixo.

Como $\mathrm{K}$ é compacto e estamos considerando os $z \in D(\infty ; r)=$ $\{W \in \mathbb{C} ;|W|>r\} \cup\{\infty\}$, então $|\zeta|<|z|$, portanto $\left|\frac{\zeta}{z}\right|<1$ e

$$
\frac{1}{\zeta-z}=-\lim _{N \rightarrow+\infty} \sum_{n=0}^{N} \frac{\zeta^{n}}{z^{n+1}}
$$

As funções $\sum_{n=0}^{N} \frac{5^{n}}{z^{n+I}}$ satisfazem $(1.1 .2)$, portanto

$$
h(z)=0 \text { em } D(\infty ; r)
$$

logo $h(z)=0$ em $v_{j} \cdot$

$$
\text { Assim, de (I.1.2) segue-se }
$$

$(1.1 .6)$

$$
h(z)=0 \text { para todo } z \in S^{2} \backslash K \text {. }
$$

Agora, escolhemos intervalos da reta orientados $\gamma_{I}, \ldots, \gamma_{n}$ em $\Omega \backslash K \quad\left[\gamma_{1}, \ldots, \gamma_{n}\right\}$ é um caminho poligonal fechado en $\Omega / K$ contendo $K$ no seu interior. Con $f \in A(\Omega)$, temos que para toda $z K$ vale a fór mula de Cauchy.

$$
f(z)=\sum_{j=1}^{n} \frac{I}{2 \pi i} \int_{\gamma_{j}} \frac{f(\zeta)}{\zeta-z} d \zeta
$$

Integrando (I.1.7) em relação a $u$ temos: 


$$
\int_{K} f(\zeta) d \mu(\zeta)=\int_{K} d \mu(\zeta)\left[\sum_{j=1}^{n} \frac{I}{2 \pi I} \int_{Y_{j}} \frac{f(w)}{w-z} d w\right] .
$$

Pelo Teorema de Fubini

$$
\int_{K} f(\zeta) d \mu(\zeta)=\sum_{j=I}^{n} \frac{I}{2 \pi I} \int_{Y_{j}} f(w)\left[\int_{K} \frac{d u(\zeta)}{w-\zeta}\right] d w
$$

Usando (I.1.4) obtemos

$$
\int_{K} f d \omega=\sum_{j=1}^{n} \frac{I}{2 \pi i} \int_{\gamma_{j}} f(w) h(w) d w_{0}
$$

Como cada $Y_{j}$ é um intervalo em $S^{2} \backslash K$, então $h(w)=0$ para to $w \in r_{j}$ com $j=1, \ldots, n$. Portanto

$$
\int_{K} f d \mu=0
$$

Logo, está demonstrado (1.1.3).

o seguinte caso particular é de especial interesse,

Teorema 1.2 - Seja $K \subset \mathbb{C}$ um conjunto compacto, $S^{2} \backslash K$ conexo e $f \in A(\Omega)$, onde $\Omega \supset K, \Omega$ aberto. Então existe uma sequência de polinômios $\left\{P_{n}\right\}$ tal que $P_{n}(z) \rightarrow f(z)$ uniformemente em $K$.

Prova - Temos que $S^{2} \backslash K$ possui uma só componente, então precisamos de somente um ponto $a_{j}$ para aplicaro Teorema I.1.1, e podemos considerar $a_{j}=\infty$ o Teorema I.1.1 implica $f \in R(K)$, isto é, existe uma sequência $\left\{P_{n}\right\}$ de funçôes racionais com polo no infinito, logo, os $\mathrm{P}_{\mathrm{n}}$ são polinomios, tal que $P_{n}(z) \rightarrow f(z)$ uniformemente em $K$. 
Observação - O resultado precedente é falso para todo compacto $K \subset C$ tal que $S^{2} \backslash K$ é não conexo. Porque neste caso $S^{2} \backslash K$ possui uma componente limitada $\mathrm{V}$

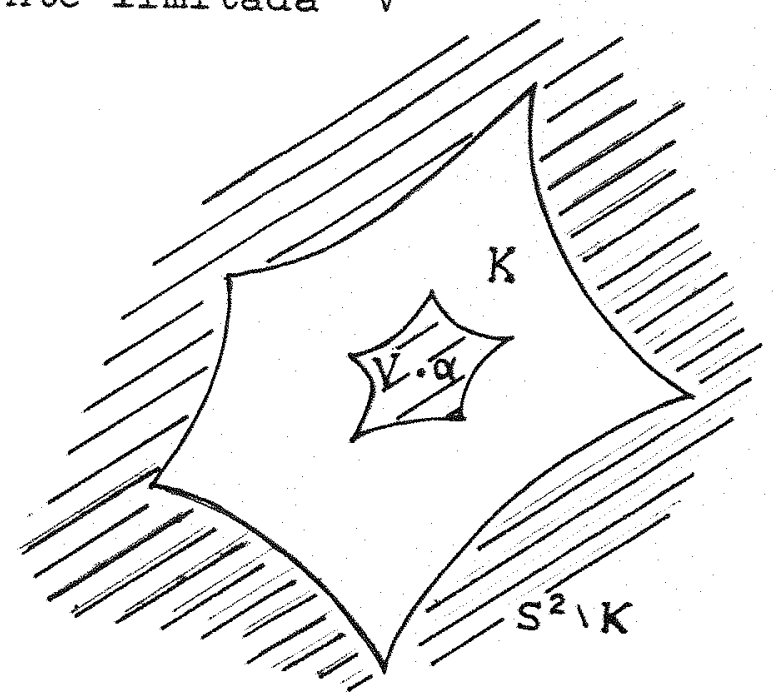

Escolha $a \in V$ e defina

$$
f(z)=\frac{1}{z-a}
$$

e $\quad m=\operatorname{máx}\{|z-a| ; z \in K\}$.

Seja P um polinômio tal que

$$
|P(z)-f(z)|<\frac{I}{m} \text { para todo } z \in K
$$

Então,

(I.1.8) $\quad|(z-\alpha) P(z)-I|<I$ para todo $z \in K$.

A desigualdade(I.1.8)é válida se $z \in O V$ e como $\overline{\mathrm{V}}$ é compacto, pelo princípio do módulo máximo,(I.1.S)é válido para todo $z \in V$. Então, fazendo $z=a$, obtemos $I<1$. Logo a aproximação uniforme não é possível. 


\section{Lemas}

Lema I.2.1- Seja $D$ um disco aberto de raio $r>0, E \subset D, E$ compac to e conexo tal que $\Omega=S^{2} \backslash E$ tambëm ë conexo, e diam $E \geq r$. Entr̃o existe uma função $g \in H(\Omega)$ e uma constante $b$, com a seguinte propriedade: Se

$$
Q(\zeta, z)=g(z)+(\zeta-b) g^{2}(z)
$$

as desigualdades

$$
|Q(\zeta, z)|<\frac{100}{r}
$$

$$
\left|Q(\zeta, z)-\frac{1}{z-\zeta}\right|<\frac{1000 r^{2}}{|z-\zeta|^{3}}
$$

são válidas para todo $z \in \Omega$ e para todo $\zeta \in D$.

Prova - Vamos supor que o centro de D está na origem, i.e., $D=D(O ; r)$

Sendo $\Omega$ e $S^{2} \backslash \Omega$ conexos, então $\Omega$ é simplesmente conexo (note que $\infty \in \Omega$ ). Pelo Teorema da Aplicação Riemann existe uma aplicação conforme $F$ de $U$ o discomitärio so bre $\Omega$ tal que $F(0)=\infty$. Então $F$ tem uma expressão da forma

$$
F(w)=\frac{a}{w}+\sum_{n=0}^{+\infty} c_{n} w^{n} \quad(w \in U) .
$$

Defina

$$
(I .2 .5) \quad g(z)=\frac{1}{a} F^{-1}(z) \quad(z \in \Omega)
$$

onde $F^{-1}: \Omega+U$ é a aplicação inversa de $F$ e seja 
$(1.2 .6)$

$b=\frac{1}{2 \pi i} \int_{\Gamma} z g(z) d z$

onde $r=C(O, r)$ estä positivamente orientado.
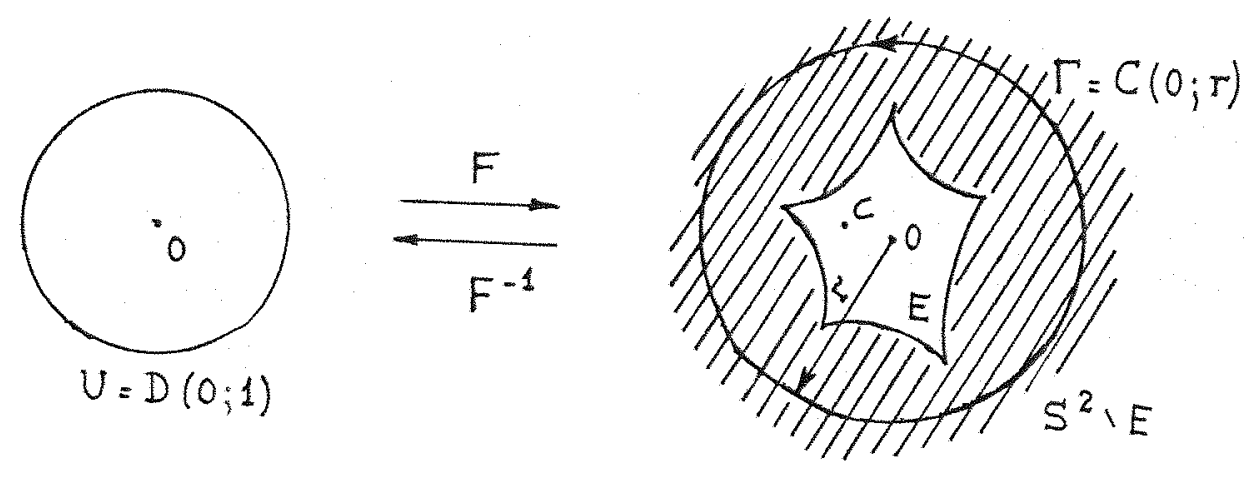

Escolha $c \in E$, então $F-c$ não possui zero em $U$.

Por (I.2.4) temos $\frac{a}{F-C} \in \mathbb{8}$, onde $\&$ é a classe de todas as $F \in A(U)$ que são $1-1$ em $U$ e satisfazem:

$$
f(0)=0 \text { e } f^{\prime}(0)=1
$$

De fato

e

$$
f(w)=\frac{1}{F(w)-c}=\frac{1}{\frac{1}{w}+\frac{1}{a} \sum_{n=0}^{+\infty} c_{n} w^{n}-c}
$$

$$
f^{\prime}(w)=\frac{+\frac{1}{w^{2}}-\frac{1}{a} n_{=1}^{+\infty} n c_{n}{ }^{n-1}}{\left[\frac{1}{w}+\frac{1}{a} \sum_{n=0}^{+\infty} c_{n} w^{n}-c \mid 2\right.}
$$

Então $f(0)=0$ e $f^{\prime}(0)=1$. Portanto $f(w)=\frac{a}{F(w)-c)} \in s$. Temos, usado o seguinte teorema se $w_{1}$, $w_{2} \notin \frac{F}{a}$ (U) então $\left|w_{1}-w_{2}\right| \leq A$. Teorema I.2.1 - Se $1 / F \in S, w_{1} \in F(U)$ e $w_{2} \in F(U)$, então $\left|w_{1}-w_{2}\right| \leq 4$. Prova: - Seja $f=1 /\left(F-w_{1}\right)$, entāo $f \in g$, dai $f(U) \supset D\left(0 ; \frac{1}{4}\right)$ (Ver 6 , pag. 278, teorema 14.14) de modo que a imagem de U sob F - w 1 contém todos os pontos w tal que $|w|>4$. Desde que $w_{1}-w_{2} \in F(U)$, temos $\left|w_{1}-w_{2}\right|>4$.

Logo dian $\left(\left(\frac{F}{A}\right)(U)\right)^{c}=\sup \left\{\left|w_{1}-w_{2}\right| ; w_{1}, w_{2} \in\left(\left(\frac{F}{a}\right)(U)\right)^{c}\right\} \leq 4$ 
Como $E \subset(F(U))^{C}$, então diam $E \leq 4|a|$. Como diam $E \geq r$, segue-se que

$$
|a| \geq \frac{r}{4}
$$

Sendo g uma aplicação conforme de $\Omega$ sobre o disco $D\left(0 ; \frac{1}{|a|}\right),(1.2 .7)$ mostra que

$(1.2 .8)$

$$
|g(z)|=\frac{1}{|a|}\left|F^{-1}(z)\right|<\frac{4}{r} \quad(z \in \Omega)
$$

e como I é um caminho em $\Omega$, de comprimento $2 \pi r$, de (1.2.6)

$$
|b| \leq \frac{I}{2 \pi} \int_{I}|z g(z)||d z|
$$

usando (I.2.8) tem-se

$$
|\mathrm{b}|<4 \mathrm{r} \text {. }
$$

Se $\zeta \in D$, então $|\zeta|<r$, portanto (T.2.1), (I.2.8)e (1.2.9) implicam

$$
|Q(\zeta, z)|=\left|g(z)+(\zeta-b) g^{2}(z)\right|<\frac{4}{r}+5 r\left(\frac{16}{r^{2}}\right)<\frac{100}{r} .
$$

Assim

$$
|Q(\zeta, z)|<\frac{100}{r}
$$

e (1.2.2) está demonstrado.

$$
\begin{array}{ll}
\text { Fixemos } & \zeta \in D \text { e seja } z=F(w) . \\
\text { Temos } & g(z)=g(F(w))=\frac{I}{a} F^{-1}(F(w))=\frac{w}{a}
\end{array}
$$

então $z g(z)=\frac{w F(w)}{a}$. 
Por outro lado, de

$$
w F(w)=a+\sum_{n=0}^{+\infty} c_{n} w^{n+1} \quad(w \in U)
$$

temos que $\mathrm{wF}(\mathrm{w}) \rightarrow \mathrm{a}$ quando $\mathrm{w} \rightarrow 0$, então $\mathrm{zg}(\mathrm{z}) \rightarrow \mathrm{I}$ quando $z \rightarrow \infty$. Daf, g possui uma expansão da forma:

$$
\begin{aligned}
& g(z)=\frac{1}{z-\zeta}+\frac{\lambda_{2}(\zeta)}{(z-\zeta)^{2}}+\frac{\lambda_{3}(\zeta)}{(z-\zeta)^{3}}+\ldots, \text { se }|z-\zeta|>2 r \\
& \text { Seja } I_{0}=C(0 ; R) \text { com raio } R>r \text {, positivamente }
\end{aligned}
$$

orientado.

Então

$$
\begin{gathered}
\frac{I}{2 \pi i} \int_{I_{0}}(z-\zeta) g(z) d z=\frac{I}{2 \pi i} \int_{I_{0}} d z+\lambda_{2}(\zeta) \cdot \frac{I}{2 \pi i} \int_{I_{0}} \frac{I}{z-\zeta}+ \\
\quad+\lambda_{3}(\zeta) \cdot \frac{1}{2 \pi i} \int_{I_{0}} \frac{d z}{(z-\zeta)^{2}}+\cdots
\end{gathered}
$$

Já que $\int_{\Gamma_{0}} d z=0$ e $\int_{\Gamma_{0}} \frac{d z}{(z-\xi)^{n}}=0$ para todo $n \geq 2$, pois $d z e \frac{d z}{(z-\zeta)^{n}}$ são formas diferenciais exatas; e como $\int_{I_{0}} \frac{d z}{z-\zeta}=2 \pi i \operatorname{Ind}_{\delta_{0}}(\zeta)=2 \pi i$ onde $\operatorname{Ind}_{\sigma_{0}}(\zeta)$ é o fndice de $I_{0}$ em relação a $\zeta$ que é igual a l, então

$$
\begin{aligned}
\lambda_{2}(\zeta) & =\frac{I}{2 \pi i} \int_{I_{0}}(z-\zeta) g(z) d z \\
& =\frac{1}{2 \pi i} \int_{I_{0}} z g(z) d z-\zeta \frac{1}{2 \pi i} \int_{I_{0}} g(z) d z .
\end{aligned}
$$


Sendo $\frac{I}{2 \pi i} \int_{I_{0}} g(z) d z=\operatorname{Res}(g ; \zeta)=1, \quad$ tem-se

$$
\lambda_{2}(\zeta)=b-\zeta
$$

Agora consideremos a função

$$
\varphi(z)=\left[Q(\zeta, z)-\frac{1}{z-\zeta}\right](z-\zeta)^{3}
$$

De (I.2.1) temos que

$$
\varphi(z)=\left[g(z)+(\zeta-b) g^{2}(z)-\frac{1}{(z-\zeta)}\right](z-\zeta)^{3}
$$

Substituindo o valor de $\lambda_{2}(\zeta)$ em (I.2.11), chegamos a

$$
\varphi(z)=\lambda_{3}(\zeta)+\frac{(\zeta-b)^{3}}{(z-\zeta)}+\frac{\lambda_{3}(\zeta)}{(z-\zeta)^{3}}+\cdots
$$

Daí, $|\varphi(z)|=\left|\lambda_{3}(\zeta)\right|$ quando $z++\infty$, istoé, é limitada quando $z \rightarrow+\infty$. Portanto possui uma singularidade removível $\mathrm{em}$ $+\infty$.

$$
\text { Se } z \in \Omega \cap D \text {, então }|z-\zeta|<2 r \text {. Assim(1.2.2) e }
$$

\section{(1.2.1.3) $1 \mathrm{mplicam}$}

$$
(1.2 .14) \mid\left(\varphi(z)|=|\left(Q(5, z)-\frac{I}{z-5}\right)(z-5)^{3}\left|<8 r^{3}\right| Q(\zeta, z) \mid+4 r^{2}<1000 r^{2} .\right.
$$

Então, pelo princípio do módulo máximo

$$
\left|Q(\zeta, z)-\frac{1}{z-\zeta \mid}\right|<\frac{1000 r^{2}}{|z-\zeta|^{3}} \text { para toda } z \in \Omega .
$$

Logo (1.2.3) está provado. 
Lema 2 - Seja $f \in C_{C}^{1}\left(\mathbb{R}^{2}\right)$ e consideremos o operador de CauchyRiemann

$$
\bar{\partial}=\frac{1}{2}\left(\frac{\partial}{\partial x}+i \frac{\partial}{\partial y}\right)
$$

Então é válida a "fórmula de Cauchy" dada por:

$$
f(z)=-\frac{1}{\pi} \int_{\mathbb{R}^{2}} \frac{(\bar{\partial} f)(\zeta)}{\zeta-z} d \xi d \eta \quad(\zeta=\xi+i \eta)
$$

Prova - Defina $(r, \theta)=f\left(z+r e^{i \theta}\right)$ com $r>0$ e $\theta \in \mathbb{R}$. Se $\quad \zeta=z+r e^{i \theta}$, da regra da cadeia temos que

$$
\begin{aligned}
& \frac{\partial \varphi}{\partial r}=\frac{\partial f}{\partial x} \cdot \frac{\partial x}{\partial r}+\frac{\partial f}{\partial y} \cdot \frac{\partial y}{\partial r} \\
& \frac{\partial \varphi}{\partial \theta}=\frac{\partial f}{\partial x} \cdot \frac{\partial x}{\partial \theta}+\frac{\partial f}{\partial y} \cdot \frac{\partial y}{\partial \theta}
\end{aligned}
$$

Então $\quad \frac{\partial \varphi}{\partial r}+\frac{i}{r} \frac{\partial \varphi}{\partial \theta}=\left(\frac{\partial f}{\partial x}+i \frac{\partial f}{\partial y}\right) e^{-i \theta}$.

Portanto

$$
(\bar{\partial} f)(\zeta)=\frac{e^{i \theta}}{2}\left[\frac{\partial \varphi}{\partial r}+\frac{i}{r} \frac{\partial \varphi}{\partial \theta}\right]
$$

$$
\text { e } \quad \frac{\bar{\partial} f(\zeta)}{\zeta-z}=\frac{I}{2 r}\left[\frac{\partial \varphi}{\partial r}+\frac{i}{r} \frac{\partial \varphi}{\partial \theta}\right], \quad d \operatorname{Cdn}=r d r d \theta
$$

Então, o lado direito de(1.2.10)é igual ao limite, quando $\varepsilon \rightarrow 0$ de

$$
-\frac{I}{2 \pi} \int_{\varepsilon}^{+\infty} \int_{0}^{2 \pi}\left[\frac{\partial \varphi}{\partial r}+\frac{i}{r} \frac{\partial \varphi}{\partial \theta}\right] d \theta d r
$$

Como para cada $r>0, \varphi$ é uma função periódica, com período 
$2 \pi, \quad$ então

$$
\int_{0}^{2 \pi} \frac{I}{r} \frac{\partial \varphi}{\partial \theta} d \theta=0
$$

Por outro lado

$$
\int^{+\infty} \frac{\partial \varphi}{\partial r}(r, \theta) d r=\lim _{M \rightarrow+\infty} \int_{\epsilon}^{M} \frac{\partial \varphi}{\partial r}(r, \theta)=-\varphi(\epsilon, \theta)+\lim _{M \rightarrow+\infty} \varphi(M, \theta)
$$

como $\varphi \in C_{C}^{1}\left(\mathbb{R}^{2}\right)$ implica $\varphi(M, \theta)=0 \quad \forall M>0$ suficientemente grande. Então

$$
\int_{\epsilon}^{+\infty} \frac{\partial \varphi}{\partial r}(r, \theta) d r=-\varphi(\varepsilon, \theta)
$$

Portanto

$$
-\frac{1}{2 \pi} \int_{0}^{2 \pi} d \theta \int_{\varepsilon}^{+\infty} \frac{\partial \varphi}{\partial r} d r=\frac{1}{2 \pi} \int_{0}^{2 \pi} \varphi(\varepsilon, \theta) d \theta
$$

Como $(\varepsilon, \theta) \rightarrow f(z)$ uniformemente em $\theta$ quando $\varepsilon \rightarrow 0$, tem-se

$$
\lim _{r \rightarrow 0}-\frac{1}{2 \pi} \int_{0}^{2 \pi} d \theta \int_{\varepsilon}^{+\infty} \frac{\partial \varphi}{\partial r} d r=\frac{1}{2 \pi} \int_{0}^{2 \pi} f(z) d \theta=f(z) .
$$

Assim, (I.2.16) está provado.

\section{O Teorema de Mergelyan}

Teorema 3.1 - Seja $K \subset \mathbb{C}$ um compacto tal que $K^{C}$ é conexo. Se $f \in C(K) e f \in A\left(K^{\circ}\right)$ e se é dado $\&>0$, então existe um polinômio $P$ tal que

$$
|f(z)-P(z)|<\varepsilon \text { para todo } z \in K
$$


Prova - Sendo $\mathbb{C}$ um espaço de Hausdorff localmente compacto, o teorema de extensão de Tietze (Ver 6 , pag 385) implica que existe uma função $F \in C_{C}(C)$ tal que $F(X)=f(x)$ para toda $x \in K$. Então, assuma que $f \in C_{C}(\mathbb{C})$ e $f \in A\left(K^{0}\right)$.

Para todo $\delta>0, \quad$ seja

$$
w(\delta)=\sup _{\left|z_{1}-z_{2}\right| \leq \delta}\left|f\left(z_{1}\right)-f\left(z_{2}\right)\right|
$$

Como $f$ é uniformemente contínua, temos

$$
\lim _{\delta 10} w(\delta)=0
$$

Agora, consideremos um $\delta$ fixo. Provaremos que existe um polinômio $P$ tal que

$$
|f(z)-P(z)|<10.000 w(\delta) \quad \text { para todo } z \in K
$$

Então, de (I.3.1) o teorema estaria demonstrado.

Passo 1. Da convolução de $f$ com uma identidade aproximada suave A com suporte pequeno, constrói-se uma função aproximada $\Phi$ de classe $\quad C_{c}^{I}\left(\mathbb{R}^{2}\right)$ a qual é igual a $f$ num dado subconjunto de $k$.

Para mostrar isto, provaremos a seguinte

Afirmação - Dado $\delta>0$, existe uma função $\Phi \in \mathrm{C}_{\mathrm{C}}^{I}\left(\mathbb{R}^{2}\right)$ tal que para todo z
$(1.3 .3)$
$|f(z)-\Phi(z)| \leq w(\delta)$,
$(1.3 .4)$

$$
|(\bar{\partial} \Phi)(z)|<\frac{2 w(\delta)}{\delta}
$$


$(1.3 .5)$

$$
\Phi(z)=-\frac{1}{\pi} \int_{X} \int_{X} \frac{(\bar{\delta} \Phi(\zeta)}{(\zeta-z)} d \xi d \eta \quad \zeta=\xi+i \eta
$$

onde $X=\left\{z \in \operatorname{supp} \Phi ; \operatorname{dist}\left(z, K^{c}\right) \leq \delta\right\}$

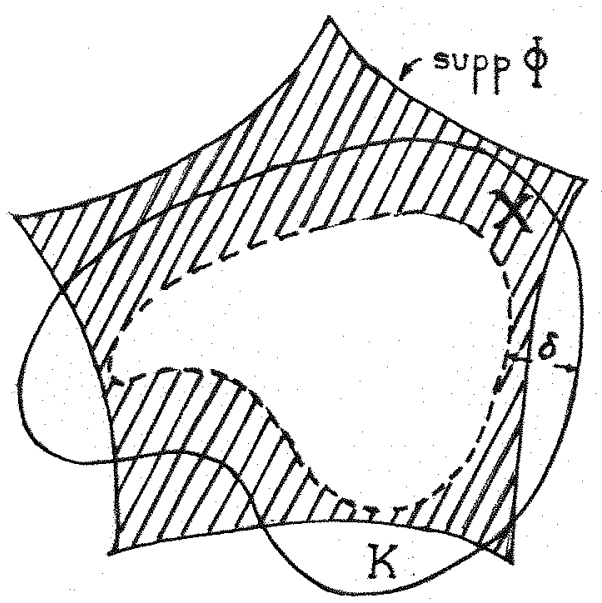

Prova - Seja a função $a: \mathbb{R} \rightarrow \mathbb{R}$ dada por

(I. 3.6)

$$
a(r)=\left\{\begin{array}{lll}
0 & \text { se } r>\delta \\
\frac{3}{\pi \delta^{2}}\left(1-\frac{r^{2}}{\delta^{2}}\right)^{2}, & \text { se } 0 \leq r \leq \delta
\end{array}\right.
$$

e defina

$(1.3 .7)$

$$
A(z)=a(|z|)
$$

para todo $z \in C$. Então, $A \in C_{C}^{1}\left(\mathbb{R}^{2}\right)$.

Agora, mostraremos que

(1.3.8)

$$
\int_{\mathbb{R}^{2}} A=1
$$

$$
\int_{\mathbb{R}^{2}} \overline{\partial A}=0
$$


(I. 3.10)

$$
\iint_{\mathbb{R}^{2}}|0 \mathrm{~A}|=\frac{24}{158}<\frac{2}{8}
$$

Prova de (I.3.8):

Usando coordenadas polares, temos que

$$
\int_{\mathbb{R}^{2}} A=\int_{0}^{\delta} \int_{0}^{2 \pi} a(r) r d r d \theta+\int_{\delta}^{+\infty} \int_{0}^{2 \pi} a(r) r d r d \theta=
$$

$\operatorname{De}(1.3 .6)$

$$
\begin{gathered}
\int_{\mathbb{R}^{2}} A=\int_{0}^{\delta} \int_{0}^{2 \pi} a(r) r d r d \theta= \\
=\frac{3}{\pi \delta^{2}} \int_{0}^{\delta} \int_{0}^{2 \pi}\left(1-\frac{r^{2}}{\delta^{2}}\right)^{2} r d r d \theta=\frac{6}{\delta^{2}} \int_{0}^{\delta}\left(1-\frac{r^{2}}{\delta^{2}}\right)^{2} r d r= \\
=\frac{6}{\delta^{2}} \cdot\left(\frac{r^{2}}{2}-\frac{r^{4}}{2 \delta^{2}}+\frac{r^{6}}{\delta^{4}}\right) \int_{0}^{\delta}=1 .
\end{gathered}
$$

Prova de (1.3.9):

$$
\begin{aligned}
& \iint_{\mathbb{R}^{2}} \bar{\partial}=\iint_{|z|>\delta} \delta A+\iint_{|z| \leq \delta} \delta_{A}=\iint_{|z| \leq \delta} \bar{\delta}, \text { pois } \\
& A(z)=0 \text { para todo }|z|>\delta \\
& \text { Como } \overline{\partial A}=\frac{1}{2} e^{i \theta}\left(\frac{\partial}{\partial r}+\frac{i}{r} \frac{\partial}{\partial \theta}\right) a(r)=\frac{1}{2} e^{i \theta} a^{\prime}(r) \text {, então } \\
& \iint_{\mathbb{R}^{2}} \overline{\partial A}=\frac{1}{2} \int_{0}^{\delta} \int_{0}^{2 \pi} e^{i \theta} a^{\prime}(r) r d r d \theta=\frac{1}{2} \int_{0}^{\delta} r a^{\prime}(r) d r \int_{0}^{2 \pi} e^{i \theta} d \theta=0
\end{aligned}
$$


Prova de (1.3.10):

$$
\begin{aligned}
& \text { Defina }(r, \theta)=A\left(z+r e^{i \theta}\right) \text {. Da regra da cadeia, tem-se } \\
& \left(\frac{\partial A}{\partial r}+\frac{i}{r} \frac{\partial A}{\partial \theta}\right)=\left(\frac{\partial A}{\partial x}+i \frac{\partial A}{\partial y}\right) e^{-i \theta}
\end{aligned}
$$

portanto $\overline{\partial A}=\frac{e^{i \theta}}{2}\left(\frac{\partial A}{\partial r}+\frac{i}{r} \frac{\partial A}{\partial \theta}\right)$.

Como $\frac{\partial A}{\partial \theta}=0$, então $\overline{\partial A}=\frac{1}{2} e^{i \theta} \frac{\partial A}{\partial r}$. Mas $\frac{\partial A}{\partial r}=a^{\prime}(r)$

e sendo

$$
a^{\prime}(r)=-\frac{12 r}{\pi \delta^{4}}\left(1-\frac{r^{2}}{\delta^{2}}\right)<0, \text { para todo } r \in[0, \delta]
$$

então $\left|\frac{\partial A}{\partial r}\right|=-a^{\prime}(r)$ e $|\overline{\partial A}|=-\frac{a^{\prime}(r)}{2}$ se $r \in[0, \delta]$. Se $r>\delta$, temos que $|\bar{\partial} A|=0$. Portanto

$$
\begin{gathered}
\iint_{\mathbb{R}^{2}}|\bar{\delta} A|=\frac{1}{2} \int_{0}^{\delta} \int_{0}^{2 \pi}-a^{\prime}(r) r d r d \theta= \\
=\frac{6}{\pi \delta^{4}} \int_{0}^{\delta} \int_{0}^{2 \pi}\left(r^{2}-\frac{r^{4}}{\delta^{2}}\right) d r d \theta=\left.\frac{12}{\delta^{4}}\left[\frac{r^{3}}{3}-\frac{r^{5}}{5 \delta^{2}}\right]\right|_{0} ^{\delta}=\frac{24}{15 \delta}<\frac{2}{\delta} .
\end{gathered}
$$

Agora defina:

$$
(1.3 .11) \Phi(z)=(f * A)(z)=\iint_{\mathbb{R}^{2}} f(z-\zeta) A(\zeta) d \xi d \eta=\iint_{\mathbb{R}^{2}} A(z-\zeta)(\zeta) d \xi d \eta \text {. }
$$

Desde que supp $f$ e supp A são compactas, então supp $\Phi$ é compacto. Por outro lado usando (I.3.8), tem-se

$$
\Phi(z)-f(z)=\iint_{\mathbb{R}^{2}}[f(z-\zeta)-f(z)] A(\zeta) d \xi d \eta
$$


Como $A(\zeta)=0$ se $|\zeta|>\delta$, então

$$
\begin{aligned}
|\phi(z)-f(z)| & =\mid \iint_{|\zeta| \leq \delta}[f(z-\zeta)-f(z) A(\zeta) d \xi d \eta \mid \leq \\
& \leq \iint_{|\zeta| \leq \delta}|f(z-\zeta)-f(z)||A(\zeta)| d \xi d \eta
\end{aligned}
$$

Mas $|f(z-\zeta)-f(z)| \leq w(\delta) \quad$ e por (I.3.8)

$$
|\phi(z)-f(z)| \leq w(\delta) \text { para todo } z \in \mathbb{C} \text {. }
$$

Logo, está demonstrado (I.3.3)

Agora, dado que $A \in C_{C}^{1}\left(\mathbb{R}^{2}\right)$, temos que existe $\frac{\partial A}{\partial x}$ e $\frac{\partial A}{\partial y}$ e são contínuas. Daí a última expressão em (I.3.11) pode-se diferenciar sob o sinal da integral, obtendo-se

(1.3.13)

$$
(\bar{\partial} \Phi)(z)=\iint_{\mathbb{R}^{2}}(\bar{\delta} A)(z-\zeta) f(\zeta) \mathrm{d} \xi \mathrm{d} \eta
$$

$$
=\iint_{\mathbb{R}^{2}} f(z-\zeta)(\bar{\zeta} A)(\zeta) d \xi d \eta
$$

Usando $(1.3 .9)$ segue-se

Então

$$
(\bar{\delta})(z)=\iint_{\mathbb{R}^{2}} f(z-\zeta)(\bar{\partial} A)(-\xi) d \zeta d \eta
$$

$|(\bar{\Phi})(z)| \leq \iint_{\mathbb{R}^{2}}|\hat{x}(z-\zeta)-f(z)||\bar{\partial} A(\zeta)| d \xi d \eta \leq w(\delta) \iint_{\mathbb{R}^{2}}|(\bar{\delta} A)(\zeta)| d \xi d \eta<\frac{2}{\delta} w(\delta)$ 
e (1.3.4) está provado.

\section{Escrevendo}

$$
\Phi_{X}(z)=\iint_{\mathbb{R}^{2}} A_{X}(z-\zeta) f(\zeta) d \xi d \eta \text { e } \Phi_{y}(z)=\iint_{\mathbb{R}^{2}} A_{y}(z-\zeta) f(\zeta) d \xi d \eta
$$

temos que $\Phi_{x}$ e $\Phi_{y}$ são contínuas, então $\Phi \in C_{C}^{1}\left(\mathbb{R}^{2}\right)$. Daí, pelo Lema I.2.2, aplicado a $\Phi$

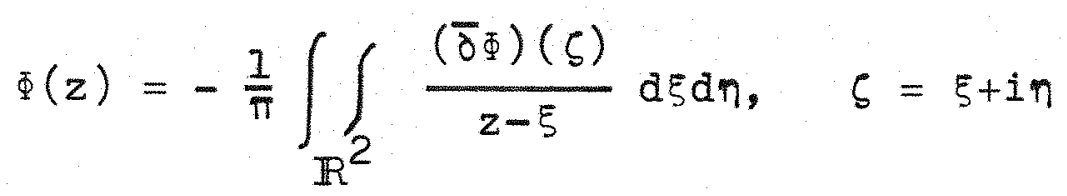

Assim, (I.3.5) segue-se se provamos que $\bar{\gamma}=0$ em $G$, onde

$$
G=\left\{z \in K ; \operatorname{dist}\left(z, K^{c}\right)>\delta\right\}
$$

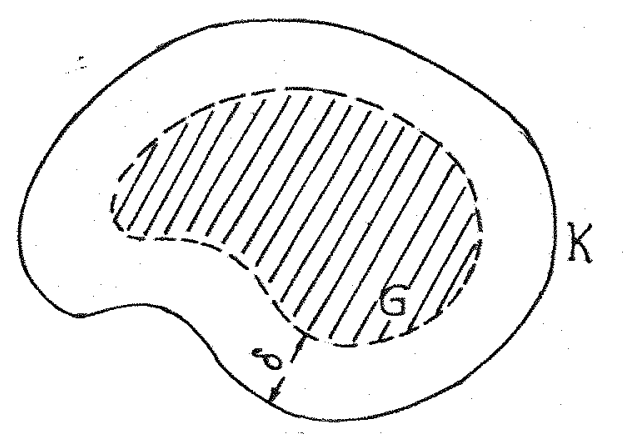

Provaremos isto, mostrando que

$$
\phi(z)=f(z) \quad(z \in G) ;
$$

observe que $\bar{\partial} f=0$ em $G$, já que $f \in A\left(K^{\circ}\right)$ e $G \subset K$. De fato, seja $z \in G$, então $z-\zeta \in K^{0}$ para todo $\zeta$ com $|\zeta|<\delta$. A propriedade do valor médio para funçoes harmônicas dá, para a primeira equação em (1.3.11). 
$(1.3 .15)$

$$
\Phi(z)=\int_{0}^{\delta} a(r) r d r \int_{0}^{2 \pi} f\left(z-r e^{i \theta}\right) d \theta=
$$

$$
=2 \pi f(z) \int_{0}^{\delta} a(r) r d r=f(z) \int_{0}^{\delta} \int_{0}^{2 \pi} a(r) r d r d \theta=f(z) \iint_{\mathbb{R}^{2}} A=f(z)
$$

para todo $z \in G$. Como $\mathbb{R}^{2}=G \cup X U(\text { supp } \Phi)^{C}$ e $\bar{\partial} \Phi=0$ em $(\operatorname{supp} \Phi)^{C}$, então

$$
\Phi(z)=-\frac{I}{\pi} \iint_{X} \frac{(\bar{\delta} \Phi)(\zeta)}{(z-\zeta)} \mathrm{d} \xi \mathrm{d} \eta
$$

onde $X=\left\{Z\right.$ supp $\left.\phi ; \operatorname{dist}\left(Z, K^{C}\right)<8\right\}$. Logo, a afirmaçâa estä provada.

Passo 2. Dividimos $X$ supp $\bar{\delta} \Phi$ em pequenas peças e aproximamos $\frac{I}{z-\zeta}$ localmente em relação a $\zeta$ e globalmente em relação a $z$, por combinações lineares

$$
Q(\zeta, z)=\sum_{i} c_{i}(\zeta) h_{i}(\zeta)
$$

de funções holomorfas sobre $K$, com coeficientes contínuos $c_{i}(\zeta)$.

Então, pelo Teorema de Runge, é suficiente aproximar $\Phi$ por uma função holomorfa numa vizinhança de $K$.

Como $X$ é compacto, e não se estende sobre $K$ mais que una distância $\delta$ de $\partial \mathrm{K}$, existe um número finito de discos abertos $D_{1}, D_{2}, \ldots, D_{n}$ tal que $D_{j}=D\left(x_{j} ; 2 \delta\right)$ com centro $x_{j} \notin K$ e $X \subset \bigcup_{j=1}^{n} D_{j}$. Como $S^{2} \backslash K$ é conexo, então cada $x_{j}$ pode se ligar ao $\infty$ por um caminho poljgonal em $S^{2} \backslash K$ sem auto-intersegões. Daí, cada disco $D_{j}$ contém um compacto $E_{j}$ tal que $\operatorname{diam}\left(E_{j}\right) \geq 2 \delta$, de modo que $S^{2} \backslash E_{j}$ é conexo e $E_{j} \cap K=\phi$ para toda j. 


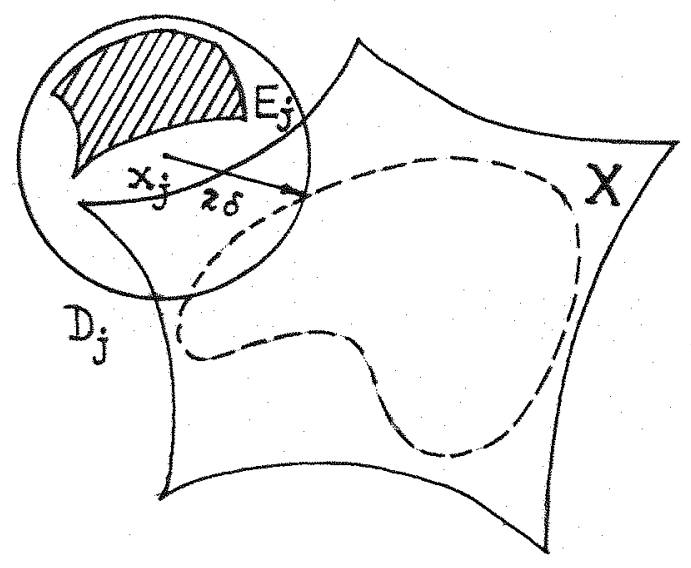

Aplicando o Lema 1.2 .1 com $r=2 \delta$, existem funções $g_{j} \in A\left(S^{2} \backslash E_{j}\right)$ e constantes $b_{j}$ de maneira que as desigualdades

$$
\begin{gathered}
\left|Q_{j}(\zeta, z)\right|<\frac{50}{\delta} \\
\left|Q_{j}(\zeta, z)-\frac{I}{z-\zeta}\right|<\frac{4000 \delta^{2}}{|z-\zeta|^{3}}
\end{gathered}
$$

são válidas para todo $z \notin E_{j}$ e $\zeta \in D_{j}$, se

$$
Q_{j}(S, z)=g_{j}(z)+\left(\zeta-b_{j}\right) g_{j}^{2}(z)
$$

Seja $\Omega=\left(\bigcup_{j=1}^{n} E_{j}\right)^{c}$. Então $\Omega$ é aberto e $K \subset \Omega$.

$$
\text { Seja } X_{I}=X \cap D_{I} \text { e } x_{j}=\left(X \cap D_{j}\right) \backslash\left(\bigcup_{i=1}^{n} x_{i}\right) \text { para }
$$

todo $2 \leq j \leq n$.

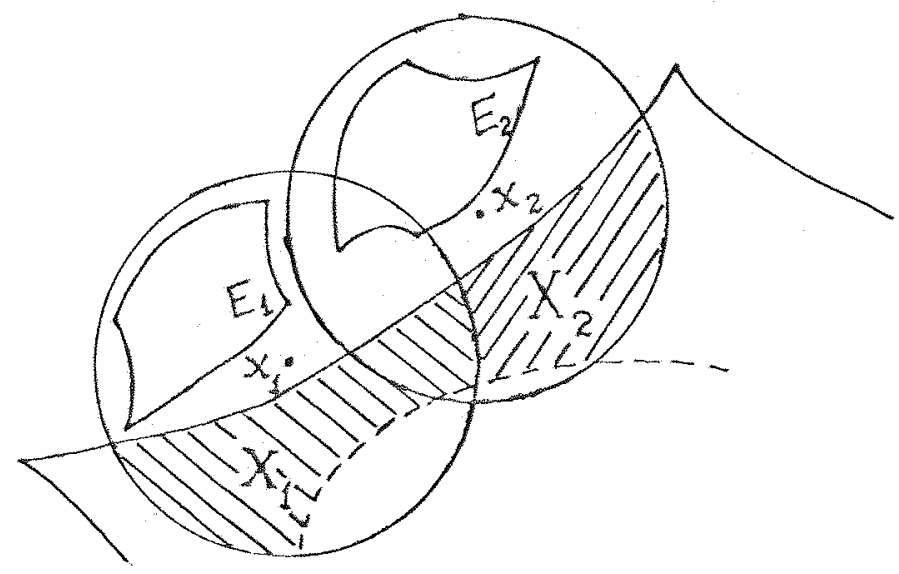


Definamos

$$
R(\zeta, z)=Q_{j}(\zeta, z) \quad\left(\zeta \in X_{j}, z \in \Omega\right)
$$

e

$$
F(z)=\frac{I}{\pi} \iint_{X} \delta \Phi(\zeta) R(\zeta, z) d \xi d \eta \quad(z \in \Omega
$$

Mostraremos que $F$ satisfaz os requerimentos estabelecidos na primeira parte da demonstração (Passo 2).

\section{Como}

$(I .3 .21) \quad F(z)=\sum_{j=1}^{n} \frac{1}{\pi} \int_{X_{j}} \bar{\delta} \Phi(\zeta) R(\zeta, z) d \xi d \eta \quad$ para toda $z \in \Omega$

então $F \in A(\Omega)$, pois (I.3.18) mostra que $F$ é uma combinação linear finita das funções $g_{j} \in \mathrm{g}_{j}^{2}$.

Portanto, de $(1.3 .20),(1.3 .4)$ e (I.3.5) temos

$(1.3 .22)$

$$
|F(z)-\Phi(z)| \leq \frac{1}{\pi} \int_{X}|(\bar{\partial} \phi)(\zeta)|\left|R(\zeta, z)-\frac{1}{z-\zeta}\right| d \xi d \eta \leq
$$

$$
s \frac{2 w(\delta)}{\pi \delta} \iint_{X}\left|R(\zeta, z)-\frac{1}{z-\zeta}\right| d s d \eta
$$

para todo $z \in \Omega$.

Agora, vejamos que a escolha da funç̃o $F$ para aproximar $\Phi$ é bastante boa. Para $\zeta \in X$ e $z \in \Omega$, as desigualodades $(1.3 .16)$ e (I.3.17) são vélidas para a função $R(\zeta, z)$, pois se $\zeta \in X$ 
então $\zeta \in X_{j}$ para algum $j$, daí segue-se $R(\zeta, z)=Q_{j}(\zeta, z)$ para todo $z \in \Omega$.

Fixemos $z \in \Omega$, e seja $\zeta=z+e^{i \theta}$. Se $0<4 \delta$, usando (1.3.16) temos:

$$
\begin{aligned}
& \iint_{X}\left|R(\zeta, z)-\frac{1}{z-\zeta}\right| d \xi d \eta<\int_{X}\left(\left|Q_{j}(\zeta, z)\right|+\left|\frac{I}{z-\zeta}\right|\right) d \xi d \eta< \\
& \quad<\int_{0}^{4 \delta} \int_{0}^{2 \pi}\left(\frac{50}{\delta}+\frac{1}{\delta}\right) \operatorname{dod} \theta=\left.2 \pi\left[\frac{50}{\delta}\left(\frac{p^{2}}{2}\right)+\theta\right]\right|_{0} ^{4 \delta}
\end{aligned}
$$

segue-se

$(\mathrm{I} .3 .23)$

$$
\iint_{X}\left|R(\zeta, z)-\frac{1}{z-\zeta}\right| d \xi d \eta<808 \pi \delta
$$

Se 248 , usando (I.3.17) temos:

$(I .3 .24) \iint_{X}\left|R(\zeta, z)-\frac{I}{z-\zeta}\right| d \xi d \eta<8000 \pi \delta^{2} \lim _{\mathbb{N} \rightarrow+\infty} \int_{4 \delta}^{N} \frac{d \rho}{\rho^{2}}=2000 \pi \delta$.

De (1.3.22)

$$
\begin{gathered}
|F(z)-\Phi(z)|<\frac{2 W(\delta)}{\delta} \iint_{X}\left|R(\zeta, z)-\frac{I}{z-\zeta}\right| d \xi d \eta= \\
=\frac{2 w(\delta)}{\delta}\left[\int_{0}^{4 \delta} \int_{0}^{2 \pi}\left|R(\zeta, z)-\frac{1}{z-\zeta}\right| d \zeta d \eta+\int_{4 \delta}^{+\infty} \int_{0}^{2 \pi}\left|R(\zeta, z)-\frac{I}{z-\zeta}\right| d \xi d \eta\right]
\end{gathered}
$$

$(z \in \Omega)$.

Substituindo $(1.3 .23)$ e (1.3.24) na expressão anterior, obte mos:

$$
|F(z)-\Phi(z)|<6000 w(\delta) \text { para todo } z \in \Omega \text {. }
$$


Desde que $F \in H(\Omega), K \subset \Omega$ e $S^{2} \backslash K$ conexo, o Teorema de Runge (Teorema 1.2) implica que existe uma sequência de polinômios $\mathrm{P}_{n}(z) \rightarrow F(z)$ uniformemente em $K$. Portanto, para todo $z \in K$

$$
\begin{aligned}
\left|f(z)-P_{n}(z)\right| & \leq|f(z)-\phi(z)|+|\phi(z)-F(z)|+\left|F(z)-P_{n}(z)\right|< \\
& <6200 w(\delta)+\left|F(z)-P_{n}(z)\right| .
\end{aligned}
$$

Como $\mathrm{P}_{\mathrm{n}} \frac{\text { unif }}{\mathrm{K}} \mathrm{F}$, dado $\epsilon=3900 \mathrm{w}(\delta)>0$ existe $\mathrm{N}_{\mathrm{o}} \in \mathrm{N}$ tal que $n>N_{0}$ implica

$$
\left|f(z)-P_{n}(z)\right|<10000 w(\delta) \text { para todo } z \in K \text {. }
$$

Como queríamos demonstrar.

4. Extensão do Teorema de Mergelyan para Domínios Multiplamente Conexos.

Teorema 4.1 - Seja $K \subset \mathbb{C}$ um conjunto compacto tal que $S^{2} \backslash K$ possui um número finito de componentes: $G_{I}, \ldots, G_{n}, \Delta_{\infty}$. Seja $f \in C(K)$ e $f \in A\left(K^{\circ}\right)$, se $\left\{a_{j}\right\}$ é um conjunto que contém um pon to $a_{j} \in G_{j}\left(\Delta_{\infty}\right)$, dado $\varepsilon>0$ existe uma sequência de funções racionais $\left\{R_{n}\right\}$ com todos seus polos contidos no conjunto $\left\{a_{j}\right\}$ tal que $R_{n}(z)+f(z)$ uniformemente em $K$.

Prova - o passo 1 é anélogo ao do Teorema de Mergelyan no caso $s^{2} \backslash \mathrm{K}$ conexo.

Passo 2: Seja $\delta_{0}>0$ tal que $4 \delta_{0}=\underset{j=1, \ldots, n}{\min }\left\{\right.$ diam $G_{j} ; G_{j}$ é componente limitada de $\left.S^{2} \backslash K\right\}$. 
Considere $0<\delta<\delta_{0}$. Sendo $X$ compacto e como para todo $z \in X$, dist $\left(z, K^{c}\right) \leq \delta$ existe um número finito de discos abertos $D_{I}, \ldots, D_{m}$ tal que $D_{j}=D\left(x_{j}, 28\right)$ com centro $x_{j} \notin K$ tal que $x \in \bigcup_{j=1}^{m} D_{j}$

Seja $\Delta_{\infty}$ a componente não Iimitada de $S^{2} \backslash K$, então $\mathrm{x}_{j} \in \Delta_{\infty}$ pode ser Iigado ao $\infty$ por um caminho poligonal em $\Delta_{\infty}$. Assim, cada disco $D_{i}=D\left(x_{i} ; 2 \delta\right)$ com $x_{i} \in \Delta_{\infty}$ contém um compacto $E_{i}$ tal que $\operatorname{diam}\left(E_{i}\right) \geq 2 \delta$, de modo que $S^{2} \backslash E_{i}$ é conexo e $E_{i} \cap K=\emptyset$.

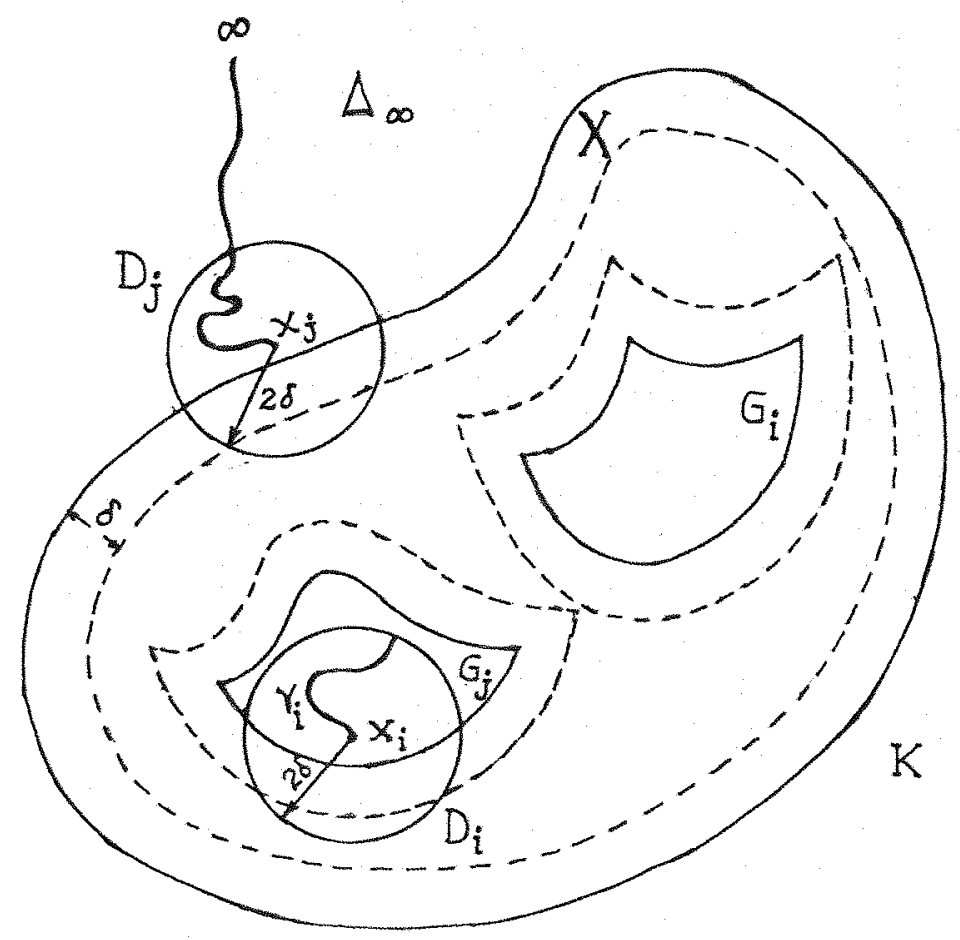

Agora, para cada componente Iimitada $G_{j}$ de $S^{2} \backslash K$, considere 0 arco de Jordan $\gamma_{i}$ contido em $G_{j} \cap D_{i}$, que parte de $x_{i}$ e que intercepta a fronteira de $D_{i}$, com comprimento $\ell\left(\psi_{i}\right)>2 \delta$. Se $\gamma_{i}^{*} \subset \gamma_{i}$ é um arco de Jordan, tal que, ainda temos $l\left(\gamma_{i}^{*}\right)>2 \delta$, considerando uma vizinhanga compacta $E_{i}$ de $\psi_{i}^{*}$ contida en 
$D_{i} \cap G_{j}$, conclui-se que: cada disco $D_{i}=D\left(x_{i} ; 2 \delta\right)$ com $x_{i} \in G_{j}$ contém un compacto $E_{i}$ tal que $\operatorname{diam}\left(E_{i}\right) \geq 2 \delta$, com $s^{2} \backslash E_{i}$ conexo e $E_{i} \cap K=\emptyset$. De modo análogo, como no Passo 2 da demonstração do Teorema de Mergelyan (Teorema I:1.3) usamos o Lema $I$ e o mesmo processo para aproximar a função $\Phi \in \mathbb{C}_{C}^{1}\left(\mathbb{R}^{2}\right)$ por uma função holomorfa $F$ no aberto $\Omega=\left(\bigcup_{j=1}^{m} E_{j}\right)^{c} \supset K$.

Pelo Teorema de Runge, sendo $F \in A(\Omega), K \in \Omega$, e dado o conjunto $\left\{\alpha_{j}\right\}$ que contém um ponto de cada componente de $s^{2} \backslash K$, existe uma sequência de funções racionais $\left\{R_{n}\right\}$ com todos os polos no conjunto $\left\{a_{j}\right\}$ tal que $R_{n}(z) \rightarrow F(z)$ uniformemente em K. Daí, segue-se o teorema. 


\section{CAPITULO II \\ A INTEGRAL DE CAUCHY \\ FORMULAS DE PLEMELJ}

Neste capitulo apresentaremos os elementos básicos da teoria da integral de Cauchy.

I. Definição e Algumas Propriedades da Integral de Cauchy

Seja $\Omega \subset C$ um aberto Iimitado com fronteira $\Omega=\Gamma \in C^{1}$ e e seja $f \in C(\Gamma)$

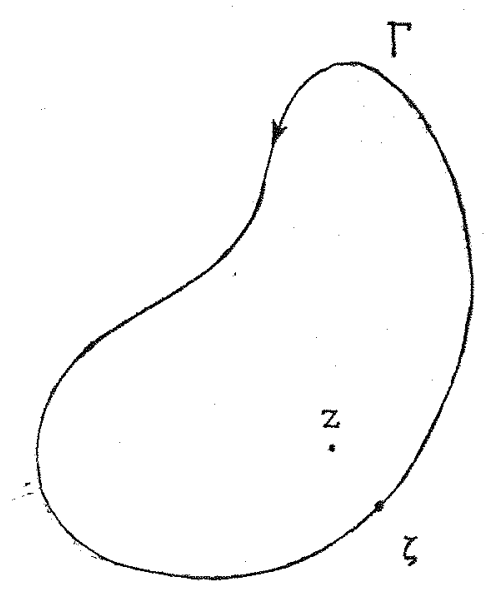

Defina

$(I I .1 .1)$

$$
w(z):=\frac{I}{2 \pi i} \int_{\Gamma} \frac{f(\zeta)}{\zeta-z} d \zeta, \quad z \notin \Gamma .
$$

A integral (II.I.I) è chamada de integral de cauchy, a qual $\vec{e}$ analitica por partes com salto na curva $\Gamma$, i.e., $w_{f} \in A\left(\Gamma^{c}\right)$. Escreveremos $w=\left(w_{f}^{i}, w_{f}^{e}\right)$ onde $w_{f}^{i}=\left.w\right|_{\Omega} e w_{f}^{e}=\left.w\right|_{\Omega} c$.

Com o propösito de dar o significado à integral (II.I.I) no caso $z=\zeta \in \Gamma$, consideremos una certa classe de funções sobre 
$\Gamma$.

Definição - A função $f(z)$ é chamada Hölder continua e escrevemos $\epsilon H(\Gamma)$, se satisfaz

$(I I . I .2) \quad\left|f\left(z^{\prime}\right)-f\left(z^{\prime \prime}\right)\right| \leqslant k(f)\left|z-z^{\prime}\right|^{\alpha}, \quad 0<\alpha \leqslant I$.

para qualquer par de pontos $z^{\prime}, z^{\prime \prime} \in \Gamma$, onde $\alpha$ e k(f) são ditas expoente de Hôlider e a constante de Hölder da função f(z), respectivamente.

Então, se $f \in H(\Gamma)$, a integral tipo Cauchy sobre $\Gamma$

$(I I .1 .3)$

$$
w_{f}(z)=\frac{1}{2 \pi i} \int \frac{f(\zeta)}{\zeta-z} d \zeta
$$

existe no sentido do valor principal de Cauchy para todos os va lores $z \in \Gamma$ (para $z \notin \Gamma$ a integral existe no sentido comum). Isto $\vec{e}$.

$$
W_{f}(z)=\frac{I}{2 \pi i} \lim _{\varepsilon \rightarrow 0} \int_{\Gamma_{\varepsilon}} \frac{f(\zeta)}{\zeta-z} d \zeta \quad, z \in \Gamma
$$

onde $\Gamma_{\varepsilon}=\Gamma-l$, e é um arco cujos pontos extremos $\zeta^{\prime}$ e $\zeta^{\prime \prime}$ satisfazem a condição $\left|z-\zeta^{\prime}\right|=\left|z-\zeta^{\prime \prime}\right|=\varepsilon, \quad z \in \ell$.

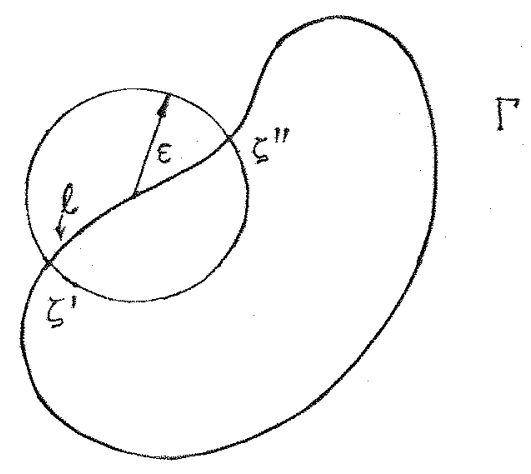


Para provar esta afirmação representemos $w_{f}(z)$ na forma

$$
w_{f}(z)=\frac{I}{2 \pi i} \int_{\Gamma} \frac{f(\zeta)-f(z)}{\zeta-z} d \zeta+\frac{f(z)}{2 \pi i} \int_{\Gamma} \frac{d \zeta}{\zeta-z}
$$

onde a primeira integral existe como integral imprópria em vista da condição $f \in H(\Gamma)$. Usando os fatos, $\left|\zeta^{\prime}-z\right|=\left|\zeta^{\prime \prime}-z\right|$ e que - ângulo $B(s)=\arg \left(\zeta^{\prime}-z\right)-\arg \left(\zeta^{\prime \prime}-z\right)$ tende para $+\pi$ quando $\varepsilon \rightarrow 0$, tem-se

$$
\int_{\Gamma} \frac{d \zeta}{\zeta-z}=\lim _{\varepsilon \rightarrow 0} \ln \frac{\zeta^{\prime}-z}{\zeta^{\prime \prime}-z}=\pi i
$$

Assim,

(II. I.4)

$$
w_{f}(z)=\frac{1}{2 \pi i} \int_{\Gamma} \frac{f(\zeta)-f(z)}{\zeta-z} d \zeta+\frac{1}{2} f(z) .
$$

Logo estä provada a afirmação.

\section{Extensão da função $f(\zeta)$ numa vizinhança do arco $\ell<\Gamma$.}

Seja $\Gamma$ um arco suave em $\mathbb{C}$ definida pela equação:

$z=\zeta(s), s \in\left[0, s_{\Gamma}\right]$, onde $s \vec{e}$ o comprimento de $\Gamma$. Sejam $\zeta_{0}=\zeta\left(s_{0}\right)$ e $\zeta=\zeta(s)$ pontos de $\Gamma, \alpha_{0}$ un ângulo agudo, $0<\alpha_{0}<\frac{\pi}{2}$, e seja $\theta=\theta(s) \circ$ ângulo que formam a tangente a $\Gamma$ no ponto $\zeta(s)$ e o eixo ox. Como $\Gamma \in C^{1}, \theta(s) \vec{e}$ uma função continua.

A continuidade do ângulo tangente $\theta(s)$ para $\Gamma$ implica a existência de un $\varepsilon>0$ tal que o ângulo não-obtuso $\alpha=\alpha(s)$ que formam as tangentes a $\Gamma$ nos pontos $\zeta_{0}$ e $\zeta=\zeta_{0}(s)$ não excede $\alpha_{0}$ 
quando $\left|s_{0}-s\right|<E, \dot{1}$.e.,

$(I I .2 .1)$

$$
|\alpha(s)|=\left|\theta\left(s_{0}\right)-\theta(s)\right| \leqslant \alpha_{0}<\frac{\pi}{2} \text {. }
$$

Agora considere $B(s)$ o ângulo não-obtuso que formam o segmento de retas que 1 iga os pontos $\zeta_{0}=\zeta\left(s_{0}\right)$ e $\zeta=\zeta(s)$ e a tan gente a $\Gamma$ num ponto arbitránio $\zeta_{1}=\zeta\left(s_{1}\right), s_{2} \in\left[s_{0}, s\right]$. Então, des de que $\Gamma$ contenha um ponto $\zeta_{*}=\zeta\left(s_{*}\right), s_{*} \in\left[s_{0}, s\right]$, no qual a tan gente a $\Gamma$ e paralelo a $\overline{\zeta_{0} \zeta}$, a desigualdade (II.2.1) implica que $(I I .2 .2)$

$$
\beta\left(s_{1}\right)=\left|\theta\left(s_{*}\right)-\theta\left(s_{1}\right)\right| \leqslant \alpha_{0}<\frac{\pi}{2} .
$$

Seja $r=\left|\zeta_{0}-\zeta\right|$, então $\left|\frac{d r}{d s}\right|=\cos \beta(s) e$

$$
k_{0}\left|s_{0}-s\right| \leqslant\left|\zeta\left(s_{0}\right)-\zeta(s)\right| \leqslant\left|s_{0}-s\right|
$$

onde $0<\mathrm{k}_{0}=\cos \alpha_{0}<1$. Observe que (II.2.3) é väIido para qual quer par de pontos $\zeta_{1}=\zeta\left(s_{1}\right)$ e $\zeta_{2}=\zeta\left(s_{2}\right)$ sobre $\Gamma$, não necessa riamente pröximos. Assim, fixemos $0<\alpha_{0}<\frac{\pi}{2}$ e $\varepsilon>0$ correspondente a este valor fixo $\alpha_{0}$, e considere um arco suave $\ell \subset \Gamma$ arbi trário com pontos extremos

$$
a=\zeta\left(s_{a}\right), b=\zeta\left(s_{b}\right) \quad\left(0 \leqslant s_{0}<s_{b}=s_{a}+\varepsilon\right) \text {. }
$$

Seja $\pi_{\zeta}$ un segmento de reta anbitrário que passa pelo ponto $\zeta=\zeta(s) \in \Gamma, s \in\left[s_{a}, s_{b}\right], e q u e$ forma um ângulo não-obtuso $\beta, 0<\alpha_{0}<\beta \leqslant \frac{\pi}{2}$, com a tangente a $l$ no ponto fixo $\zeta_{0}=\zeta\left(s_{0}\right)$. Agora, seja.na a normal no ponto extremo a do arco $l$ e faça uma translação paralela de $\ell$ à uma distância $\varepsilon_{0}$ à ambos lados de $\ell$, tomando os pontos extremos à direita das curvas $l^{\prime}$ e $\ell^{\prime \prime}$ sobre a 
nonmal $n_{a}$

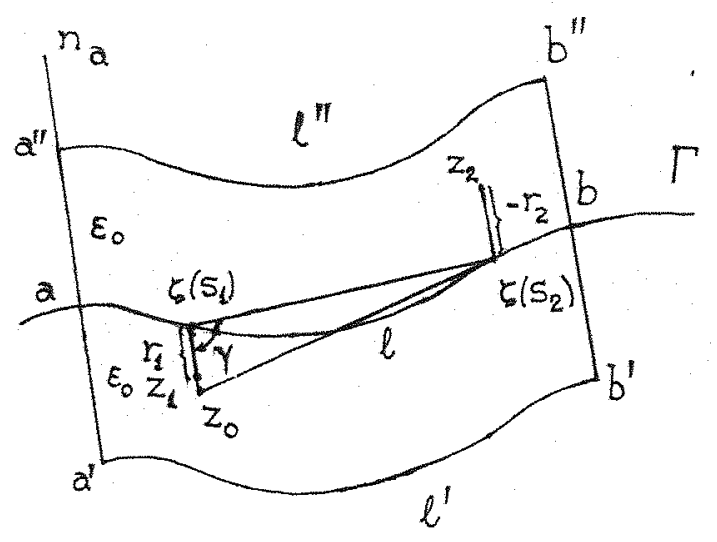

O segmento $a^{\prime} a^{\prime \prime}$ de $n_{a}$ e o segmento $b^{\prime} b^{\prime \prime}$ da reta paralela à $\mathrm{n}_{\mathrm{a}}$, com as curvas transladadas $\ell^{\prime} \mathrm{e} \ell$ "', determinam um dominio limitado $\sigma=\sigma(\ell)$, ao qual chamaremos de vizinhança standand do arco $\ell$,

Seja z $\epsilon \sigma(\ell)$ arbiträrio. Considere uma reta paralela à $\mathrm{n}_{a}$ e denote pon $\zeta=\zeta(s)$ seu ponto de intenseção com $\ell$. Seja $r=\mp|z-\zeta|$, onde o sinal mais se considera se z fica à direita do arco $\ell$ quando vamos de "a" e "b", e consideramos o sinal menos em caso contrário. Como s e r são a coordenadas generalizadas do ponto $z \in \sigma(\ell)$, então $z=z(s, r) \cdot \operatorname{seja} z_{1}=z\left(s_{1}, r_{1}\right)$, $z_{2}=z\left(s_{2}, r_{2}\right) \in \sigma(\ell)$ anbitránios, e $\rho\left(z_{1}, z_{2}\right)$ a menor distância entre as curvas coordenadas, i.e.,

$$
p\left(z_{1}, z_{2}\right)=\left|s_{1}-s_{2}\right|+\left|r_{1}-r_{2}\right| \text {. }
$$

Considere o triängulo com vértices nos pontos $\left.\zeta_{1}=\zeta_{\left(s_{1}\right.}\right)$, $\zeta_{2}=\zeta\left(s_{2}\right)$ e $z_{0}=z\left(s_{1},\left|r_{1}-r_{2}\right|\right)$; por construção

$$
\left|z_{1}-z_{2}\right|=\left|z_{0}-\zeta_{2}\right|,\left|z_{0}-\zeta_{1}\right|=\left|r_{1}-n_{2}\right| \text {. }
$$


e, por (II.2.1), o ângulo $\gamma$ no vêrtice $\zeta_{1}$ satisfaz a desigualda de $0<\frac{\pi}{2}-\alpha_{0}<\gamma \leqslant \frac{\pi}{2}$. Então

$$
\begin{aligned}
\left|z_{0}-\zeta_{2}\right|^{2} & =\left|z_{0}-\zeta_{1}\right|^{2}+\left|\zeta_{2}-\zeta_{1}\right|^{2}-2 \cos \gamma\left|\zeta_{1}-\zeta_{2}\right|\left|z_{0}-\zeta_{1}\right| \geqslant \\
& \geqslant \operatorname{sen}^{2} \frac{\gamma}{2}\left(\left|z_{0}-\zeta_{2}\right|+\left|\zeta_{2}-\zeta_{2}\right|\right)^{2},
\end{aligned}
$$

donde, por (II.2.I),

$$
\left|z_{1}-z_{2}\right|=\left|z_{0}-\zeta_{2}\right| \geqslant \sin \frac{\gamma}{2}\left(\left|r_{1}-r_{2}\right|+k_{0}\left|s_{1}-s_{2}\right|\right) \geqslant k\left(\alpha_{0}\right) \rho\left(z_{1}, z_{2}\right)
$$

onde $k\left(\alpha_{0}\right)=k_{0} \sin \frac{\gamma}{2} \geqslant \cos \alpha_{0} \operatorname{sen}\left(\frac{\pi}{4}-\frac{\alpha_{0}}{2}\right)>0$. Assim,

(II.2.4) $\quad k_{p}\left(z_{1}, z_{2}\right) \leqslant\left|z_{1}-z_{2}\right| \leqslant \rho\left(z_{1}, z_{2}\right), \quad k=k\left(\alpha_{0}\right)>0$.

Tomando $\rho\left(z_{1}, z_{2}\right) \geqslant\left\{\left|s_{1}-s_{2}\right|,\left|r_{1}-r_{2}\right|\right\}$, por (II.2.4), temos

(II.2.5) $\quad\left|s_{1}-s_{2}\right| \leqslant \frac{1}{\bar{k}}\left|z_{1}-z_{2}\right|,\left|r_{1}-r_{2}\right| \leqslant \frac{1}{k}\left|z_{1}-z_{2}\right|$.

Agora suponha que $f(\zeta) \in H(l)$, sobre 0 arco $l \in \Gamma$, onde $\Gamma$ é uma curva suave arbitrária, tal que $s_{b}-s_{a}=\varepsilon$, onde $a=\zeta\left(s_{a}\right)$ e $b=\zeta\left(s_{b}\right)$ são os pontos extremos de $l$, e $\varepsilon$ se escolhe de manei ra que (II.2.I) é vâlido. Seja $f(z)=f(\zeta)$, onde $\zeta=-\zeta(s) \in \ell \cap L$ e I é a reta que passa por z paralela à normal $\mathrm{n}_{\mathrm{a}} \mathrm{em}$ a.

Então, $f(z) \in H[\sigma(l)]$ para a função que estende $f$ definida em $l \subset \Gamma$ para a vizinhança standard $\sigma(l)$ de $\ell$. De fato, usan do as desigualdades (II.2.3) e (II.2.4) obtemos

$$
\begin{aligned}
& \left|f\left(z_{1}\right)-f\left(z_{2}\right)\right|=\left|f\left(\zeta_{1}\right)-f\left(\zeta_{2}\right)\right| \leqslant k(f)\left|\zeta_{1}-\zeta_{2}\right|^{\alpha} \leqslant \\
& \quad \leqslant k(f)\left|s_{1}-s_{2}\right|^{\alpha} \leqslant k(f) k_{0}^{-\alpha}\left|z_{1}-z_{2}\right|^{\alpha}
\end{aligned}
$$


isto $\vec{e}$,

(II.2.6) $\left|f\left(z_{1}\right)-f\left(z_{2}\right)\right| \leqslant \tilde{k}(f)\left|z_{j}-z_{2}\right|^{\alpha}$, onde $\tilde{k}(f)=k(f) k_{0}^{-\alpha}$

3. Comportamento da Integral de Cauchy nas Proximidades da Curva de Integração.

Para estudar como se comporta a integral de cauchy numa vizinhança da curva $T$ represente $w_{f}$ na forma

$(I I .3 . I) w_{f}(z)=\frac{I}{2 \pi i} \int_{\Gamma} \frac{f(\zeta)-f(z)}{\zeta-z} d \zeta+\frac{f(z)}{2 \pi i} \int_{\Gamma} \frac{d \zeta}{\zeta-z}, z \in \sigma(l)$

onde $f(\zeta) \in H\left(L_{0}\right), L_{0} \subset \Gamma$, e $f(\zeta)$ é integrävel sobre $\Gamma-L_{0}, e f(z)$ ë a extensão da $f(\zeta)$ numa vizinhança standard $\sigma(\ell)$ de um arco ar bitránio $l \subset L_{0}$ com pontos extremos $a_{\ell}=t\left(s_{a}\right)$ e $b_{\ell}=t\left(s_{b}\right)$. Seja

$$
F(z)=\frac{I}{2 \pi i} \int_{\Gamma} \frac{f(\zeta)-f(z)}{\zeta-z} d \zeta
$$

As propriedades de $F$, estão dados pelo seguinte

Teorema II.3.I - $F(z) \in H(\sigma(l)), l \subset L_{0}$, com expoente de Hölder $\lambda=\alpha$ para $0<\alpha<1$, e $\lambda \in(0,1)$ arbiträrio para $\alpha=1$. A constante de Holden $k(F)$ àa função $F(z)$ depende de $k(f)$ sobre $L_{0}$, do valor da integral de $f(\zeta)$ sobre $\Gamma-L_{0}$, da distancia dos pontos extremos de L e da geometria de L.

Prova - Ver [4], pag 1], teonema 1. 
4. Propriedades de Fronteira da Integral de Cauchy. Fórmulas de Plemelj.

Em primeiro lugar, consideremos os valores de fronteira da integral de Cauchy $w_{f}(z)$. Para isso, tome $\zeta \in \Gamma$ e denote $w_{f}^{i}(\zeta) e w_{f}^{e}(\zeta)$ os valores limites sobre $\Gamma$ da função $w_{f}(z)$ quando a aproximação é por $\Omega$ e por $\bar{\Omega}$, respectivamente, caso existam. Suponha que $f(\zeta)$ e $H(\Gamma), L_{0} \subset \Gamma$ (arco), e que z $\in \Omega$ estä numa vizinhança $\sigma(\ell)$ do arco $l \in L_{0}$ contendo o ponto fixo $\zeta \in \Gamma$
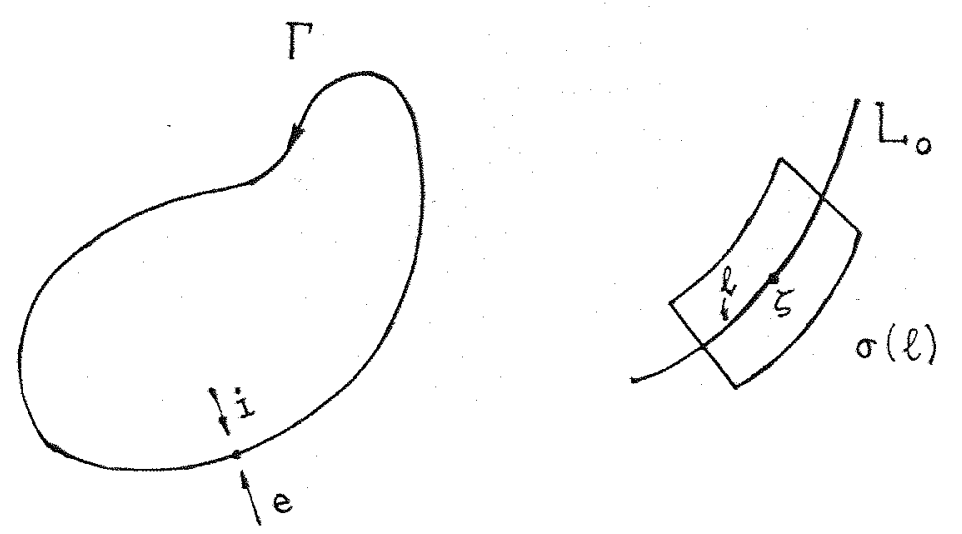

Como

$$
\frac{1}{2 \pi i} \int_{\Gamma} \frac{d \zeta}{\zeta-z}=1, \quad z \in \Omega
$$

de (II.3.1) tem-se

$(I I .4 . I) \quad w_{f}(z)=\frac{I}{2 \pi i} \int_{\Gamma} \frac{f(\zeta)}{\zeta-z} d \zeta=\frac{I}{2 \pi i} \int_{\Gamma} \frac{E(\zeta)-f(z)}{\zeta-z} d \zeta+f(z)$

i.e, $w_{f}(z)=F(z)+f(z)$, onde $f(z) \vec{e}$ a extensão de $f(\zeta)$ nums vizinhanga $\sigma(l)$. Passando ao Iimite em (II.4.I) quando. $z \rightarrow \zeta_{0} \in \Gamma$ $(z \in \Omega)$, tem-se

$$
w_{f}^{i}\left(\zeta_{0}\right)=\frac{1}{2 \pi i} \int_{\Gamma} \frac{f(\zeta)-f(\zeta)}{\zeta-\zeta_{0}} d \zeta+f\left(\zeta_{0}\right), \quad \zeta_{0} \in \Gamma .
$$


A existência dos Iimites segue-se de que $F(z)$ (Teorema II. 3.1 )

e $f(z)$ são Hölder contínuas. Assim, pela igualdade

$$
\frac{1}{2 \pi i} \int_{\Gamma} \frac{d \zeta}{\zeta-\zeta_{0}}=\frac{1}{2}
$$

a qual segue-se de (II.I.4) para $f(z)=f(\zeta)=1$, obtemos

$$
(I I .4 .2) \quad w_{f}^{i}\left(\zeta_{0}\right)=\frac{I}{2} f\left(\zeta_{0}\right)+\frac{I}{2 \pi i} \int_{\Gamma} \frac{f(\zeta)}{\zeta-z} d \zeta \quad\left(\zeta_{0} \in \Gamma\right) .
$$

Agora, suponha que $z \in(\bar{\Omega})^{c}$, então

$$
\frac{1}{2 \pi i} \int_{\Gamma} \frac{d \zeta}{\zeta-z}=0, \quad z \in \bar{\Omega}^{c}
$$

e, em consequéncia ( $I$. $3 . I$ ) pode-se re-escrever na forma

$$
w_{f}(z)=\frac{I}{2 \pi i} \int_{\Gamma} \frac{f(\zeta)}{\zeta-z} d \zeta=\frac{1}{2 \pi i} \int_{\Gamma} \frac{f(\zeta)-f(\zeta)}{\zeta-z} d \zeta
$$

a qual, quando $z \rightarrow \zeta_{0}\left(z \in \in \bar{\Omega}^{c}\right)$, nos dä, pelas mesmas razões que anteriormente,

$(I I .4 .3) \quad w_{f}^{e}\left(\zeta_{0}\right)=-\frac{I}{2} f\left(\zeta_{0}\right)+\frac{I}{2 \pi i} \int_{\Gamma} \frac{f(\zeta)}{\zeta-\zeta_{0}} d \zeta$.

As fórmulas (II.4.2) e (II.4.3) são chamadas de förmulas de Plemelj.

observe que $w_{f}^{i} \in A(\Omega)$ e $w_{f}^{e} \in A\left(\bar{\Omega}^{c}\right)$.

Escreveremos tambëm $w_{f}^{i}$ para designar $w$ quando $z \in \bar{\Omega}$ e $w_{f}^{e}$ para designar $w$ quando $z \in \Omega^{c}$.

Sobre a curva $\Gamma$ temos o seguinte resultado (Ver [6], pag. 16, teorema 2) 
Teorema II.4.I (Privalov). Seja $\Gamma \in C^{1}$ uma curva simples fechada e $\Omega$ seu interion. Se $f \in H^{\alpha}(\Gamma), 0<\alpha \leqslant I$, então $w_{f}^{j} \in H^{\lambda}(\bar{\Omega})$ e $w_{f}^{e} \in H^{\lambda}\left(\Omega^{c}\right)$ onde $\lambda=\alpha$ se $0<\alpha<1$ e $\lambda \vec{e}$ qualquer valor em $(0,1)$ se $\alpha=1$. 


\section{CAPITULO III}

\section{NUCLEOS ORTONORMALIZADORES PARA CURVAS FECHADAS SIMPLES}

Neste capítulo definiremos um produto interno numa curva $\Gamma$ fechada simples no plano, de modo que as potências $z^{n}$, n inteiro relativo, formem um conjunto ortogonal no espaço das fun ções Hölden contínuas na curva munida do produto interno dado que denotaremos $H_{r}(\Gamma,\langle\rangle$,$) . Tambëm, exibiremos uma base ortonon$ mal para o espaço $H_{r}(\Gamma,\langle\rangle$,$) e estudaremos as propriedades do$ operador integral $\tilde{\mathbb{K}}$ que define o produto interno, assim como as de seu nücleo.

Alëm disso, vamos caracterizar o completamento do espaço $\mathrm{H}_{\Gamma}(\Gamma,\langle\rangle$,$) , analisando em que sentido a série formal de laurent$ de uma função $f \in C(\Gamma)$ converge para $f$; neste ponto a ferramenta bäsica ê o Teonema de Mergelyan para domínios multiplamente conexo.

\section{Definição e Forma Explícita do Produto Interno}

Nesta seção, nosso primeiro objetivo é definir um produ to interno sobre o espaço de funções $H(\Gamma)$, com expoente $0<\alpha \leqslant 1$, de maneina que o conjunto $\left\{z^{n}\right\}_{n=-\infty}^{+\infty}$ seja ontogonal na curva $\Gamma$. Para isso, considere $f \in H(\Gamma)$, e defina a aplicação Iinear.

$$
f \in H(\Gamma) \stackrel{w}{\longrightarrow}\left(w_{f}^{i}, w_{f}^{e}\right) \in[A(\Omega) \cap H(\bar{\Omega})] \times\left[A\left(\bar{\Omega}^{c}\right) \cap H(\Omega c)\right]
$$

Pelas fómmlas de Plemelj, seguc-se que a aplicação é injetiva. De fato, se $\zeta \in \Gamma$, temos $w_{f}^{i}(\zeta)-w_{f}^{e}(\zeta)=f(\zeta)$. 
Agora, vamos considenar os casos especiais:

i) $f(z)=z^{n}, n \in \mathbb{Z}^{+} U\{0\}$,

ii) $f(z)=z^{-n}, n \in \mathbb{Z}^{+}$.

Proposicão (III.I.I) - Se $f(z)=z^{n}, n \in \mathbb{Z}^{+} U\{0\}$ temos $w_{f}^{i}(z)=z^{n} e$ $w_{f}^{e}(z)=0$. Ese $f(z)=z^{-n}, n \in \mathbb{Z}^{+}$temos $w_{f}^{i}(z)=0$ e $w_{f}^{e}(z)=z^{-n}$.

Prova - Com efeito, se $f(z)=z^{n}, n \in \mathbb{Z}^{+} U\{0\}$ segue $f \in A(\Omega)$. Por (II.I.I),

$$
w(z)=\frac{1}{2 \pi i} \int_{\Gamma} \frac{\zeta^{n}}{\zeta-z} d \zeta
$$

Se $z \in \Omega$, pela fönula integral de Cauchy

$$
w_{z}^{i}(z)=z^{n}
$$

ese $z \in \bar{\Omega}^{c}$, temos $\frac{\zeta^{n}}{\zeta-z}$ é analitica, logo ${ }_{z^{n}}^{e}(z)=0$

$$
\begin{gathered}
\text { No caso } f(z)=z^{-n}, n \in \mathbb{Z}^{+} \text {, temos } f \in A\left(\bar{\Omega}^{c}\right) \text {. De (III.I.I), } \\
w(z)=\frac{1}{2 \pi i} \int_{\Gamma} \frac{\zeta^{-n}}{\zeta-z} d \zeta
\end{gathered}
$$

Seja $\zeta^{\prime}=\frac{I}{\zeta}$ então $d \zeta^{\prime}=-\frac{1}{\zeta^{2}} d \zeta$ e

$$
w\left(z^{\prime}\right)=\frac{z^{\prime}}{2 \pi i} \int_{\Gamma} \frac{\zeta^{n-1}}{\zeta^{\prime}-z^{\prime}} d \zeta^{\prime}
$$

onde $\Gamma^{\prime}$ è a curva obtida de $\Gamma$ pela transfommąão $z \longrightarrow \frac{1}{z}$, no $z^{\prime}-$ plano 

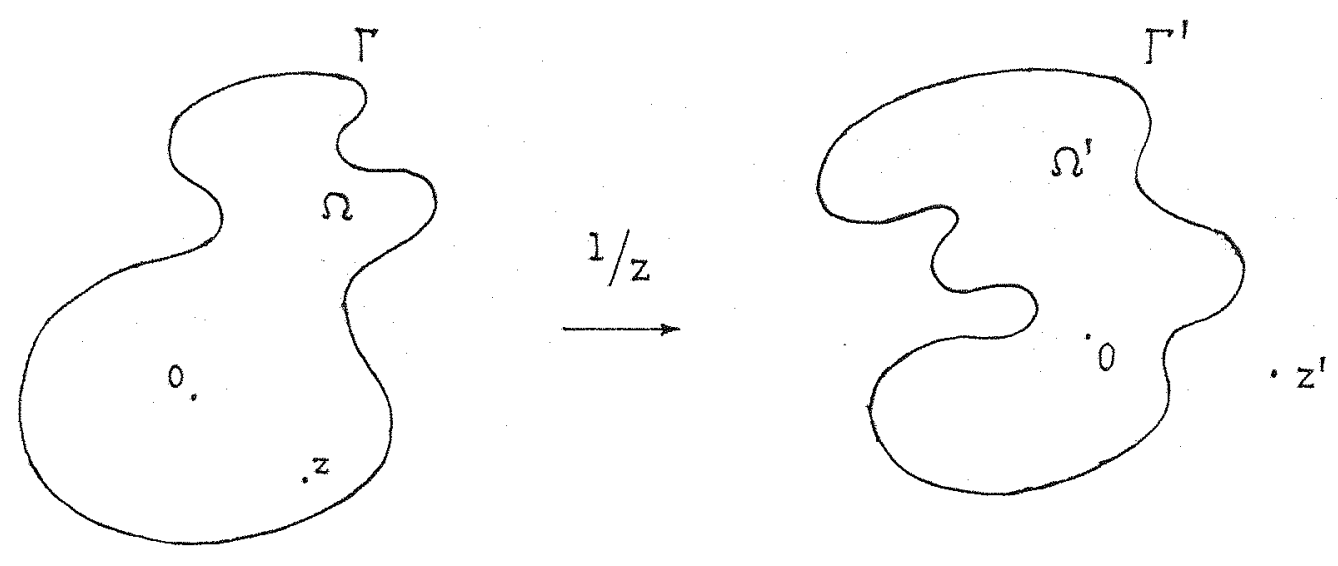

Se $z^{\prime} \in \bar{\Omega}^{c}$, temos $\frac{\zeta^{i n-I}}{\zeta^{\prime}-z^{\prime}} \vec{E}$ analitica, então ${ }_{z^{i n}}^{e}\left(z^{\prime}\right)=0$, e se $z^{\prime} \in \Omega^{\prime}$, pela fórmula integral de Cauchy $w_{z^{\prime}}^{i}\left(z^{\prime}\right)=z^{n}$. Mas $w_{z^{\prime}}^{e}\left(z^{\prime}\right)=w_{z^{n}}^{i}(z)$ e $w_{z^{\prime n}}^{i}\left(z^{\prime}\right)=w_{z^{-n}}^{e}(z)$. Como queríamos demonstrar. Note que

$$
z^{n} \stackrel{w}{\longrightarrow}\left(z^{n}, 0\right)
$$

e

$$
z^{-n} \stackrel{w}{\rightarrow}\left(0, z^{-n}\right)
$$

Suponhamos que $0 \in \Omega$. Tomemos $C_{i}=C(0 ; r) \in \Omega$ um círculo de centro 0 e raio $0<n<1, e c_{e}=c\left(0 ; n^{-1}\right)$ un círculo de centro 0 e raio $r^{2}$, com $c_{e}=\bar{\Omega}^{c}$, como na figura.

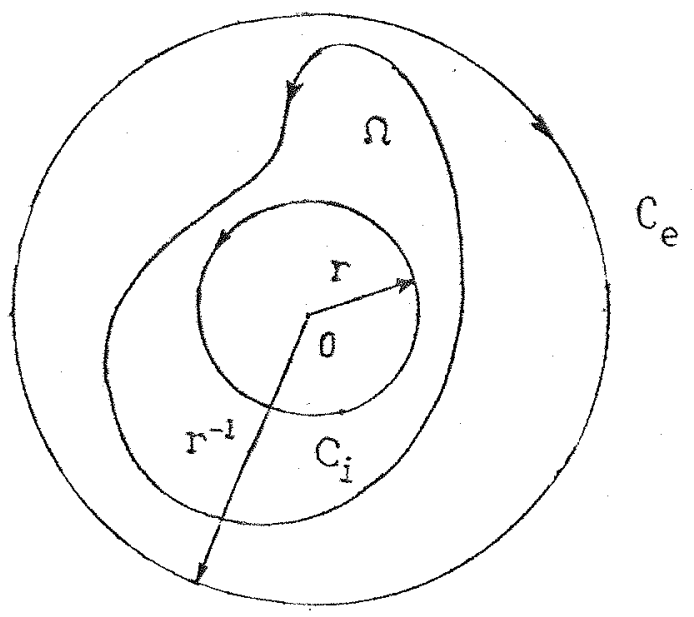




\section{Definição do Produto Interno}

Sejam $a>0$ e $b>0$ constantes. Defina a função $<,>: H(\Gamma) \times H(\Gamma) \rightarrow \mathbb{C}$ por

(III.1.1) $\left\langle E, h>:=a \oint_{C} \bar{w}_{f}^{i} \frac{i}{h} d s+b \oint_{C} \bar{w}_{f}^{e} w_{h}^{e} d s, f, h \in H(\Gamma)\right.$

onde ds é o elemento de arco, $\oint_{C_{i}}$ e $\oint_{C_{e}}$ denotam integração so bre $C_{i}$ e $C_{e}$ respectivamente, com orientação positiva.

E imediato que $\langle$,$\rangle é um produto interno em vista$ de sua definição e da injetividade da aplicação $\mathrm{w}$.

Fazendo $f(z)=z^{m}$ e $h(z)=z^{n}$, onde $m, n \in \mathbb{Z}$, temos, obviamente,

$$
\left\langle z^{m}, z^{n}\right\rangle=0 \text { se } m \neq n
$$

Desta maneira $\left\{z^{n}\right\}$ é um conjunto ortogonal em $H\left(\Gamma,<_{r}>\right)$.

Forma Explicita do produto Interno

Em $C_{i}, w_{f}^{i}(z)=\frac{1}{2 \pi i} \int_{\Gamma} \frac{f(\zeta)}{\zeta-z} d \zeta$ e $w_{h}^{i}(z)=\frac{1}{2 \pi i} \int_{\Gamma} \frac{h(\zeta)}{\zeta-z} d \zeta ;$ em

$C_{e} w_{f}^{e}(z)=\frac{1}{2 \pi i} \int_{\Gamma} \frac{f(\zeta)}{\zeta-z} d \zeta \quad$ e $w_{h}^{e}(z)=\frac{1}{2 \pi i} \int_{\Gamma} \frac{h(\zeta)}{\zeta-z} d \zeta$,

Dai 


$$
\begin{aligned}
& \langle\mathrm{f}, \mathrm{h}\rangle=\frac{1}{4 \pi^{2}}\left\{a \oint_{C_{i}} \int_{\Gamma} \frac{\overline{\mathrm{f}}(\zeta)}{(\bar{\zeta}-\bar{z})} d \bar{\zeta} \cdot \int_{\Gamma} \frac{h\left(\zeta^{\prime}\right)}{\zeta^{\prime}-z}\left(d \zeta^{\prime}\right) d s+\right. \\
& \left.+\operatorname{b\phi }_{C_{e}}\left(\int_{\Gamma} \frac{\overline{\underline{I}}(\zeta)}{\bar{\zeta}-\bar{z}} d \bar{\zeta} \cdot \int_{\Gamma} \frac{h\left(\zeta^{\prime}\right)}{\zeta^{\prime}-z} d \zeta^{\prime}\right) d s\right\}= \\
& =\int_{\Gamma} \int_{\Gamma} \bar{f}(\zeta) h\left(\zeta^{\prime}\right)\left(\int_{a c_{i}-b c_{e}} \frac{\left(\frac{1}{4 \pi^{2}}\right)}{(\bar{\zeta}-\bar{z})\left(\zeta^{\prime}-z\right)} d s\right) d \bar{\zeta} d \zeta^{\prime} .
\end{aligned}
$$

para $f, h \in H(\Gamma)$.

Agora, defina

$$
K\left(\zeta, \zeta^{\prime}\right)=\int_{a c_{i}-b c_{e}} \frac{\left(\frac{1}{4 \pi^{2}}\right)}{(\bar{\zeta}-\bar{z})\left(\zeta^{\prime}-z\right)} d s .
$$

Então

$$
\langle f, h\rangle=\int_{\Gamma \Gamma} \bar{f}(\zeta) h\left(\zeta^{\prime}\right) K\left(\zeta, \zeta^{\prime}\right) d \bar{\zeta} d \zeta^{\prime}
$$

Suponha que a curva I esteja parametrizada pelo com primento de arco $s$, i.e., $\Gamma: \zeta=\zeta(s)$;

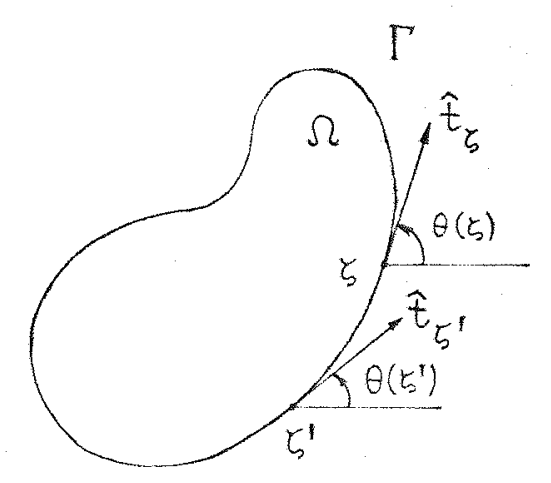

Então, $d \zeta=e^{i \theta(\zeta)} d s \quad e d \zeta^{\prime}=e^{i \theta\left(\zeta^{\prime}\right)} d s^{\prime}$, onde $\theta=\theta(\zeta)$ é ângulo entre a tangente $\bar{t}_{\zeta}$ a $\Gamma$ no ponto $\zeta$, e o eixo ox. Logo 


$$
\begin{aligned}
\langle f, h\rangle & =\iint_{\Gamma} \bar{f}(\zeta) h\left(\zeta^{\prime}\right) e^{i\left(\theta\left(\zeta^{\prime}\right)-\theta(\zeta)\right)} K\left(\zeta, \zeta^{\prime}\right) d s d s^{\prime} \\
& \text { para todo } f, h \in H(\Gamma)
\end{aligned}
$$

Seja

$\tilde{K}\left(\zeta, \zeta^{\prime}\right)=K\left(\zeta, \zeta^{\prime}\right) e^{i\left(\theta\left(\zeta^{\prime}\right)-\theta(\zeta)\right)}, \quad\left(\zeta=\zeta(s)\right.$ e $\left.\zeta^{\prime}=\zeta^{\prime}\left(s^{\prime}\right)\right)$

e defina o operador integral $\tilde{\mathbb{K}}: H(\Gamma) \rightarrow H(\Gamma)$ por

$$
(\tilde{\mathbb{K}} h)(\zeta)=\int_{\Gamma} \tilde{K}\left(\zeta, \zeta^{\prime}\right) h\left(\zeta^{\prime}\right) d s^{\prime} .
$$

Considerando o espaço $L^{2}(\Gamma)$, com a medida de Lebesgue, onde $($, ) é o produto interno usual, tem-se

$$
\left\langle f, i_{1}\right\rangle=(f, \tilde{\mathbb{K}} h) \quad f, h \in H(\Gamma)
$$

Agora, determinemos a forma geral do produto interno. Assim, seja $c_{i}=c(0, r)$ o circulo de centro na origem e raio $0<r<1$ $e z=r e^{i \theta}$

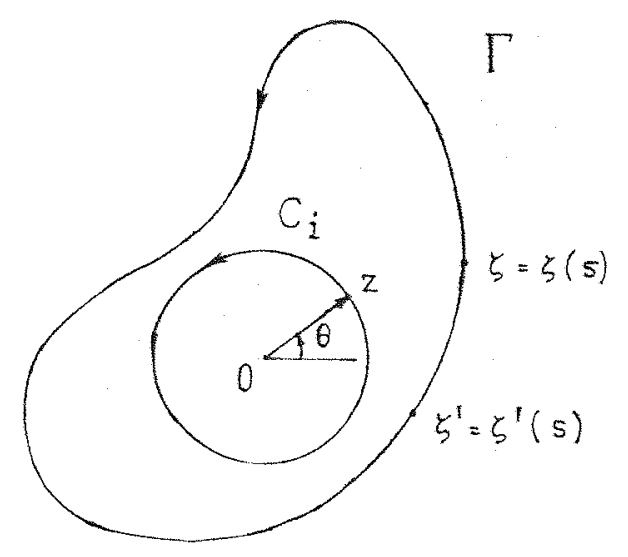

então $\bar{z}=r e^{-i \theta}, d s=r a \theta e$ 


$$
\begin{aligned}
\oint_{C_{i}} \frac{d s}{(\bar{\zeta}-\bar{z})\left(\zeta^{\prime}-z\right)} & =\int_{0}^{2 \pi} \frac{d\left(r e^{i \theta}\right)}{\left(\bar{\zeta}-r e^{-i \theta}\right)\left(\zeta^{\prime}-r e^{i \theta}\right)}=-i \int_{0}^{2 \pi} \frac{d\left(r e^{i \theta}\right)}{\left(e^{i \theta} \bar{\zeta}-r\right)\left(\zeta^{\prime}-r e^{i \theta}\right)} \\
& =-i \int_{C_{i}} \frac{d z}{\left(r^{-1} z \zeta-r\right)\left(\zeta^{\prime}-z\right)} \\
& =-i \int_{C_{i} \frac{r}{\bar{\zeta}} \frac{\left(z-\frac{r^{2}}{\bar{\zeta}}\right)\left(\zeta^{\prime}-z\right)}{d z}}
\end{aligned}
$$

Como $\frac{r^{2}}{\bar{\zeta}} \in D(0 ; r)$ e $\frac{1}{\zeta^{1}-z}$ é uma funcão analitica em $D(0 ; r), \underline{u}$ sando o teorema dos residuos, tem-se

$$
\begin{gathered}
\oint_{C_{i}} \frac{d s}{(\bar{\zeta}-\bar{z})\left(\zeta^{\prime}-z\right)}=2 \pi \frac{r}{\bar{\zeta}} \operatorname{Res} \frac{1}{\left(z-\frac{r^{2}}{\bar{\zeta}}\right)\left(\zeta^{\prime}-z\right)} ; \frac{r^{2}}{\bar{\zeta}}= \\
=2 \pi \frac{r}{\bar{\zeta}} \cdot \frac{1}{\zeta^{\prime}-\frac{r^{2}}{\bar{\zeta}}}=\frac{2 \pi r}{\bar{\zeta} \zeta^{\prime}-r^{2}} .
\end{gathered}
$$

Como

$\oint_{C_{i}} \bar{w}_{f}^{-i} w_{h}^{i} d s=\frac{1}{4 \pi^{2}} \int_{\Gamma} \int_{\Gamma} \bar{E}(\zeta) h\left(\zeta^{\prime}\right)\left(\oint_{C_{i}} \frac{d s}{(\bar{\zeta}-\bar{z})\left(\zeta^{\prime}-z\right)}\right) d \bar{\zeta} d \zeta^{\prime}$

então

$$
\oint_{c_{i}} \bar{w}_{f}^{i} w_{h}^{i} d s=\frac{r}{2 \pi} \int_{\Gamma \Gamma} \frac{\bar{f}(\zeta) h\left(\zeta^{\prime}\right)}{\left(\bar{\zeta} \zeta^{\prime}-r^{2}\right)} d \bar{\zeta} a \zeta^{\prime}
$$

Analogamente, seja $c_{e}=c\left(0 ; x^{-1}\right), \quad 0<r<1$ e $z=r^{-1} e^{i \theta}$ 


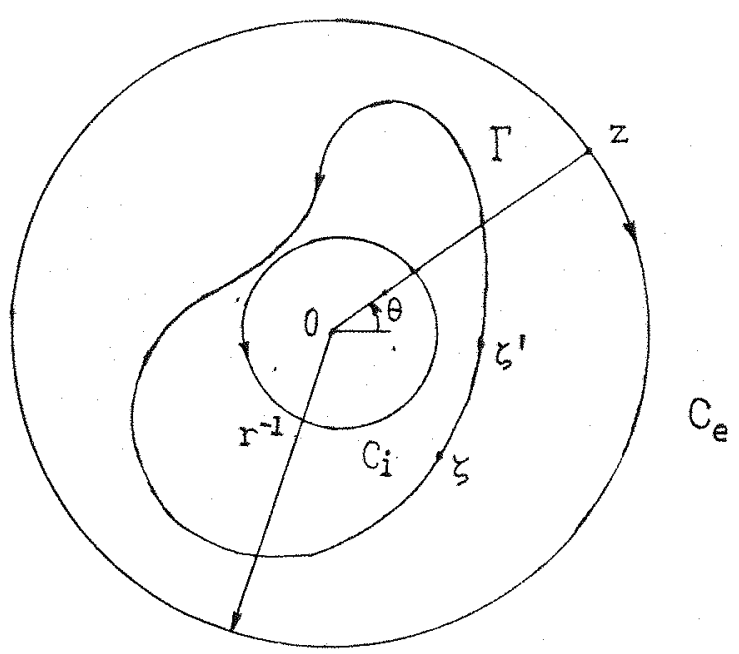

Então $\bar{z}=r^{-1} e^{-i \theta} d s=r^{-1} d \theta, \quad \theta \in[0,2 \pi]$

$$
\begin{aligned}
& \oint_{c_{e}} \frac{d s}{(\bar{\zeta}-\bar{z})\left(\zeta^{\prime}-z\right)}=-\oint_{c_{e}} \frac{d s}{(\bar{\zeta}-\bar{z})\left(\zeta^{\prime}-z\right)}=-\int_{0}^{2 \pi} \frac{r^{-1} d \theta}{\left(\bar{\zeta}-r^{-1} e^{-i \theta}\right)\left(\zeta^{\prime}-z\right)}= \\
& =-\int_{0}^{2 \pi} \frac{-i e^{-i \theta} d\left(r^{-1} e^{i \theta}\right)}{\left(\bar{\zeta}-r^{-1} e^{-i \theta}\right)\left(\zeta^{\prime}-z\right)}=+i \oint_{-c_{e}}^{r \bar{\zeta}} \cdot \frac{1}{\left(z-r^{-2} \bar{\zeta}^{-1}\right)\left(z-\zeta^{\prime}\right)} \cdot
\end{aligned}
$$

Como a função $\frac{1}{z-r^{-2} \bar{\zeta}^{-1}}$ é analittica em $\bar{\Omega}^{c}$ e $\frac{1}{z-\zeta^{1}}$ tem um polo em $z=\zeta^{\prime} \in D\left(0 ; r^{-1}\right)\left(r<\left|\zeta^{\prime}\right|<r^{-1}\right)$, pelo teorema dos residuos temos

$$
\begin{gathered}
\oint_{C_{e}} \frac{d s}{(\bar{\zeta}-\zeta)\left(\zeta^{\prime}-z\right)}=-\frac{2 \pi}{r \bar{\zeta}} \operatorname{Res}\left(\frac{1}{\left(z-\zeta^{\prime}\right)\left(z-r^{-2} \bar{\zeta}^{-1}\right)} ; \zeta^{\prime}\right)= \\
=-\frac{2 \pi}{r \bar{\zeta}} \cdot \frac{1}{\zeta^{\prime}-r^{-2} \bar{\zeta}^{-1}}=-\frac{2 \pi r^{-1}}{\bar{\zeta} \zeta^{\prime}-r^{-2}} .
\end{gathered}
$$

Sendo 


$$
\oint_{C_{e}} \bar{w}_{f}^{e} w_{h}^{e} d s=\frac{1}{4 \pi^{2}} \int_{\Gamma \Gamma} \bar{f}(\zeta) h\left(\zeta^{\prime}\right)\left(\int_{C_{e}} \frac{d s}{(\bar{\zeta}-\bar{z})\left(\zeta^{\prime}-z\right)}\right) d \bar{\zeta} d \zeta^{\prime}
$$

segue-se

$$
\oint_{c} \bar{w}_{f}^{e} w_{h}^{e} d s=-\frac{r^{-1}}{2 \pi} \int_{\Gamma} \int_{\Gamma} \frac{\bar{f}(\zeta) h\left(\zeta^{\prime}\right)}{\left(\bar{\zeta} \zeta^{\prime}-r^{-2}\right)} d \bar{\zeta} d \zeta^{\prime}
$$

Substituindo (III.1.7) e (III.1.8) em (III.1.1), obtemos a forma geral do produto interno

(III.1.9) $\langle f, h\rangle=\frac{1}{2 \pi} \iint_{\Gamma} \bar{f}(\zeta) h\left(\zeta^{\prime}\right)\left(\frac{a r}{\bar{\zeta} \zeta^{\prime}-r^{2}}-\frac{b r^{-1}}{\bar{\zeta} \zeta^{\prime}-r^{-2}}\right) d \bar{\zeta} d \zeta^{\prime}$

então

$$
K\left(\zeta, \zeta^{\prime}\right)=\frac{1}{2 \pi}\left(\frac{a r}{\bar{\zeta} \zeta^{\prime}-r^{2}}-\frac{b r^{-1}}{\bar{\zeta} \zeta^{\prime}-r^{-2}}\right)
$$

é o núcleo do operador integral IK e

$$
\tilde{K}\left(\zeta, \zeta^{\prime}\right)=\frac{1}{2 \pi}\left(\frac{a r}{\bar{\zeta} \zeta^{\prime}-r^{2}}-\frac{b r^{-1}}{\bar{\zeta} \zeta^{\prime}-r^{-2}}\right) e^{i\left(\theta\left(\zeta^{\prime}\right)-\theta(\zeta)\right)}
$$

é o núcleo do operador integral $\tilde{\mathbb{K}}$.

Tomando $\mathrm{a}=\mathrm{r}$ e $\mathrm{b}=\mathrm{r}^{-1}, 0<r<1$, sem perda de generalidade, tem-se 
$K\left(\zeta, \zeta^{\prime}\right)=\frac{1}{2 \pi}\left(\frac{r^{2}}{\bar{\zeta} \zeta^{\prime}-r^{2}}-\frac{r^{-2}}{\bar{\zeta} \zeta^{\prime}-r^{-2}}\right)=\frac{1}{2 \pi}\left(\frac{r^{2}}{\bar{\zeta} \zeta^{\prime}}\left(\frac{1}{1-\frac{r^{2}}{\bar{\zeta} \zeta}}\right)+\frac{1}{1-r^{2} \bar{\zeta} \zeta^{\prime}}\right)$

Como para todo $\zeta \in \Gamma, r<|\zeta|<r^{-1}$, então $r^{2}<|\zeta \bar{\zeta}|<r^{-2}$. Portanto $\left|\frac{r^{2}}{\zeta^{\prime} \bar{\zeta}}\right|<1$ e $r^{2}\left|\bar{\zeta} \zeta^{\prime}\right|<1$. Então

$$
\begin{aligned}
K\left(\zeta, \zeta^{\prime}\right)=\frac{1}{2 \pi}\left(\frac{r^{2}}{\bar{\zeta} \zeta^{\prime}}+\left(\frac{r^{2}}{\bar{\zeta} \zeta^{\prime}}\right)^{2}+\ldots+1+r^{2} \bar{\zeta} \zeta^{\prime}+\right. \\
\left.+\left(x^{2} \bar{\zeta} \zeta^{\prime}\right)^{2}+\ldots\right)
\end{aligned}
$$

ou seja

$$
K\left(\zeta, \zeta^{\prime}\right)=\frac{1}{2 \pi} \sum_{n=-\infty}^{+\infty} r^{2|n|}\left(\bar{\zeta} \zeta^{\prime}\right)^{n} \text { para todo }\left(\zeta, \zeta^{\prime}\right) \in \Gamma \times \Gamma
$$

Assim,

$$
\begin{aligned}
& \tilde{\mathrm{K}}\left(\zeta, \zeta^{\prime}\right)=\frac{1}{2 \pi} \sum_{n=-\infty}^{+\infty} r^{2|n|} e^{-i \theta(\zeta)}\left(\bar{\zeta} \zeta^{\prime}\right)^{\mathrm{n}} r^{i \theta\left(\zeta^{\prime}\right)} \text { para todo } \\
& \left(\zeta, \zeta^{\prime}\right) \quad \Gamma \times \Gamma .
\end{aligned}
$$

Como para todo $\zeta_{;} \zeta^{\prime} \in \Gamma$ temos $\left|r^{2} \bar{\zeta} \zeta^{\prime}\right|<1$ e $\left|\frac{r^{2}}{\bar{\zeta} \zeta^{\prime}}\right|<1$, as séries (III.1.13) e (III.1.14) convergem absoluta e uniformemente so bre $\Gamma \times \Gamma$.

Observação: O núcleo do operador integral IK se comporta como a delta de Dirac $\delta\left(\zeta-\zeta^{\prime}\right)$ quando $r \rightarrow 1$, isto é, quando $\Gamma=s^{1}$. Com efeito, de (III.1.10) temos 


$$
K\left(\zeta, \zeta^{\prime}\right)=\frac{1}{2 \pi}\left(\frac{r^{2}}{\bar{\zeta} \zeta^{\prime}-r^{2}}-\frac{r^{-2}}{\bar{\zeta} \zeta^{\prime}-r^{-2}}\right)
$$

para $a=r$ e b $=r^{-1}, 0<r<1$.

Então, se $\zeta \neq \zeta^{\prime}$ temos

$$
K\left(\zeta, \zeta^{\prime}\right) \longrightarrow 0 \quad \text { quando } r \rightarrow 1
$$

$$
\begin{aligned}
& \text { Se } \zeta=\zeta^{\prime}, \text { temos } \\
& \begin{aligned}
K(\zeta, \zeta) & =\frac{1}{2 \pi}\left(\frac{r^{2}}{1-r^{2}}-\frac{r^{-2}}{1-r^{-2}}\right) \\
& =\frac{1}{2 \pi}\left(\frac{r^{2}-r^{-2}}{2-\left(r^{2}+r^{-2}\right)}\right) \\
& =\frac{1}{2 \pi} \cdot \frac{r+r^{-1}}{r^{-1}-r}
\end{aligned}
\end{aligned}
$$

de onde $K(\zeta ; \zeta) \rightarrow+\infty$ quando $r \longrightarrow 1$.

Denote por $\mathrm{H}_{\Gamma}\left(\Gamma_{,}<,>\right)$o espaço $H(\Gamma)$ munido do produto interno $\langle$,$\rangle , quando a=r$ e $b=r^{-1}$, em $0<r<1$. Observe que $H_{r}(T,<,>)$ possui uma estrutura de álgebra sem elemento unidade. De fato, se $f, g \in A(\Omega) \cap H(\Gamma)$ temos

$$
w_{f}^{\dot{I}}=f, \quad w_{g}^{i}=g \text { em } \Omega \text { e } w_{f}^{e}=0, w_{g}^{e}=0 \text { em } \bar{\Omega}^{c}
$$

emtão,

$$
w_{f}^{i} w_{g}^{i}=f g=w_{f g}^{i} \text { em } \Omega \text { e } w_{f}^{e} w_{g}^{e}=0=w_{f g}^{e} \text { em } \bar{\Omega}^{c} \text {. }
$$


então,

$$
w_{f}^{i} w_{g}^{i}=0=w_{f g}^{i} \text { em } \Omega \text { e } w_{f}^{e} \cdot w_{g}^{e}=0=w_{f g}^{e} \text { em } \bar{\Omega}^{c} .
$$

Analogamente, se $f, g \in A\left(\bar{\Omega}^{C}\right) \cap H(\Gamma)$ temos

$$
w_{f}^{i}=0, w_{g}^{i}=0 \text { em } \Omega \text { e } w_{f}^{e}=f, w_{g}^{e}=g e, \quad \bar{\Omega}^{c}
$$

então,

$$
\begin{aligned}
& \quad w_{f}^{i} \cdot w_{g}^{i}=0=w_{f g}^{i} \text { em } \Omega \text { e } w_{f}^{e} \cdot w_{g}^{e}=f g=w_{f g}^{e} \text { em } \bar{\Omega}^{c} ; \\
& \quad \text { Por outro lado, se e é a identidade, então e }\left.\right|_{\Omega}=1 \text { e } \\
& e_{\left.\right|_{\Omega} c}=1 .
\end{aligned}
$$

Dai

$$
=w_{e}^{i}=w_{e}^{e}=1
$$

e portanto $e_{\Gamma}=\left.w_{e}^{i}\right|_{\Gamma}-\left.w_{e}^{e}\right|_{\Gamma}=0$. Mas $\left.\right|_{\Gamma}=0$, então $e \equiv 0$. Absurdo.

\section{Propriedades do operador $\mathbb{K}$}

1) $\tilde{\mathbb{K}}$ é limitado na norma $L^{2}(\Gamma)$.

De fato, pela convergência uniforme de (III.1.13) e pela compacidade de $\Gamma \times \Gamma$, existe $M<+\infty$ tal que $\left|\tilde{K}\left(\zeta, \zeta^{\prime}\right)\right|<M$

$$
\int_{\Gamma} \int_{\Gamma}\left|\tilde{K}\left(\zeta, \zeta^{\prime}\right)\right|^{2} d s d s^{\prime}<M^{2} \iint_{\Gamma} d s d s^{\prime}<+\infty
$$


2) $\overline{\mathbb{K}}$ é um operador compacto em $\mathrm{L}^{2}(\Gamma)$

Com efeito, pela convergência uniforme em $\Gamma \times \Gamma$ da série (III.1.13) de termos continuos em $\Gamma \times \Gamma$ segue $K\left(\zeta ; \zeta^{\prime}\right) \in C(\Gamma \times \Gamma)$. Combinando isto com o fato de $\Gamma$ ser compac to, concluí-se $\tilde{\mathbb{K}}$ é um operador compacto em $\mathrm{L}^{2}(\mathrm{I})$.

3) $\tilde{\mathbb{K}}$ é autoadjunto em $L^{2}(\Gamma)$.

Basta provar que

$$
\overline{\mathrm{R}\left(\zeta^{\prime}, \zeta\right)}=\tilde{\mathrm{R}}\left(\zeta, \zeta^{\prime}\right)
$$

De fato,

$$
\begin{aligned}
\overline{\tilde{K}\left(\zeta^{\prime}, \zeta\right)} & =\frac{1}{2 \pi} \sum_{n=-\infty}^{+\infty} r^{2|n|} e^{-i \theta\left(\zeta^{\prime}\right)} \overline{\left(\zeta^{\prime} \zeta\right)^{n}} e^{i \theta(\zeta)}= \\
& =\frac{1}{2 \pi} \sum_{n=-\infty}^{+\infty} r^{2|n|} e^{-i \theta(\zeta)}\left(\bar{\zeta} \zeta^{\prime}\right)^{n} e^{i \theta\left(\zeta^{\prime}\right)}=\tilde{K}\left(\zeta, \zeta^{\prime}\right) .
\end{aligned}
$$

Assim, temos as seguintes propriedades do núcleo $\tilde{\mathrm{K}}$.

1') $\tilde{K}$ é de quadrado integrável sobre $\Gamma \times \Gamma$ tal que

$$
\int_{\Gamma} \int_{\Gamma}\left|\tilde{K}\left(\zeta, \zeta^{\prime}\right)\right|^{2} d s a s^{\prime}<+\infty .
$$

$\left.2^{\prime}\right) \quad \tilde{K} \in C(\Gamma \times \Gamma):$

3') $\tilde{K}$ é hermiteano. 
Em consequêencia, o produto interno $\langle$,$\rangle é contínuo$ no sentido de $L^{2}(\Gamma)$. De fato, pela desigualdade de Cauchy-Schwarz

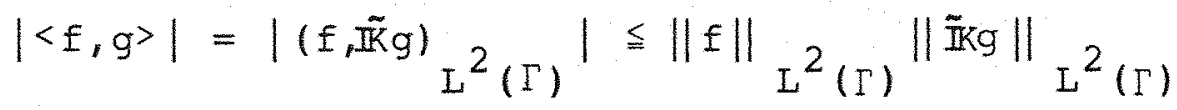

Mas

$$
\|\overline{\mathbb{K}} g\|_{L^{2}(\Gamma)} \leqq\|\tilde{\mathbb{K}}\|_{L^{2}(\Gamma)}\|g\|_{L^{2}(\Gamma)}
$$

Portanto

$$
\|\langle f, g\rangle\| \leqslant\|\tilde{\mathbb{K}}\|_{L^{2}(\Gamma)}\|f\|_{L^{2}(\Gamma)}\|g\|_{L^{2}(\Gamma)}
$$

Logo $L^{2}(\Gamma)$ está contiáo no completamento de $\left.\mathrm{H}_{\Gamma}(\Gamma,<\rangle,\right)$.

3) Uma Base Ortonormal para o Espaco $H_{r}(\Gamma,<,>)$

Temos definido em $H(\Gamma)$ o produto interno $\langle$,$\rangle que$ satisfaz

$$
\langle f, g\rangle=(f, \tilde{\mathbb{K}} g) \quad f, g \in H(\Gamma)
$$

Vamos mostrar que o conjunto $\left\{z^{m_{m}}\right\}_{m=-\infty}^{+\infty}$ é ortogonal em relação a este produto interno. Primeiro, analisemos o eEeito do operador $\tilde{\mathbb{K}}$ nas funçōes $z{ }^{m}, m \in Z$. 
Multiplicando (III.1.13) por $\zeta^{\mathrm{m}}$, obtemos:

(III.3.1) $\quad \zeta^{\prime m_{\tilde{K}}}\left(\zeta, \zeta^{\prime}\right)=\frac{1}{2 \pi} \sum_{n=-\infty}^{+\infty} r^{2|n| \bar{\zeta}^{n} \zeta^{\prime}(n+m)} e^{i\left(\theta\left(\zeta^{\prime}\right)-\theta(\zeta)\right)}$

Integre ambos membros de (III.3.1) em relação a $\zeta$ ' na curva $\Gamma$. Pela convergência uniforme da série em (III.3.1) em $\Gamma \times \Gamma$ combinado com o fato

$$
\int_{\Gamma} \zeta^{(n+m)} d \zeta^{\prime}= \begin{cases}0, & \text { se } n+m \neq-1 \\ 2 \pi i, & \text { se } n+m=-1\end{cases}
$$

Segue-se

$(I I I .3 .2)\left[\tilde{K}\left(z^{m}\right)\right](\zeta)=\int_{\Gamma} \tilde{K}\left(\zeta, \zeta^{\prime}\right) \zeta^{\prime m} d s=i r^{2|m+1|} \bar{\zeta}^{-(m+1)} e^{-i \theta(\zeta)}$
$\left\langle z^{n}, z^{m}=i r^{2|m+1|} \int_{\Gamma} \bar{\zeta}^{[n-(m+1)] d \bar{\zeta}}\right.$

mas

$$
\int_{\Gamma} \bar{\zeta}^{[n-(m+1)]} d \bar{\zeta}= \begin{cases}0, & \text { se } n \neq m \\ -2 \pi i, & \text { se } n=m\end{cases}
$$

então

$$
<z^{n}, z^{m}>=2 \pi r^{2|n+1|} \delta_{m, n}
$$


onde $\delta_{m, n}$ é o delta de Krönecker.

Em conseqüencia, o conjunto $\left\{z^{\mathrm{m}}\right\}$ é ortogonal em relạ̄ão ao produto interno $<,>$ com

$$
\left\|z^{m}\right\|_{<,>}=\sqrt{2 \pi} r^{|m+1|}, m \in \mathbb{Z}
$$

\section{Tomando}

(III.3.3)

$$
\theta_{\mathrm{m}}=\frac{\mathrm{z}^{\mathrm{m}}}{\sqrt{2 \pi} \mathrm{r}^{|\mathrm{m}+1|}}
$$

obtemos que o conjunto $\left\{\theta_{m}\right\}$ é ortonormal em $H_{r}(\Gamma,<,>)$.

Agora provaremos que o conjunto ortonormal $\left\{\theta_{m}\right\}$ é maximal em $H_{I}(\Gamma,<,>)$. De fato, se $f \in H_{r}(\Gamma,<,>)$ então $f \in C(\Gamma)$, e pelo Teorema de Merǵglyan (I.5.1) existe uma sequuência de funcões racionais $\left\{\phi_{\mathrm{p}} \text { : } \phi_{\mathrm{p}} \text { tem polo em } O \in \Omega \text { e em }{ }^{\infty}\right\}_{\mathrm{p}=1}^{\infty}$ tal que $\phi_{p} \stackrel{C(\Gamma)}{\rightarrow f}$ quando $p \rightarrow+\infty, i . e . .$, dado $\varepsilon>0$ existe uma fun cão racional com polos em 0 e no $\infty$ tal que

$$
\|\phi-f\|_{\infty}<\frac{\varepsilon}{\sqrt{L}\left(\|\tilde{\mathbb{K}}\|_{L^{2}(\Gamma)}+I\right)^{1 / 2}}
$$

onde $\|\cdot\|_{\infty}$ denota a norma do supremo em $\mathrm{T}$ e I é o comprimento da curva $\Gamma$. $\lambda_{\mathrm{m}}^{\varepsilon} \in \mathbb{C}$

Dai existe $\mathrm{F} \subset \mathbb{Z}$ finito, tal que $\phi=\sum_{\mathrm{m} \in \mathrm{F}} \lambda_{\mathrm{m}}^{\varepsilon_{\mathrm{m}}}$ onde 
Por outro lado, a convergência uniforme sobre a cur va $\Gamma$ implica a convergência em $L^{2}(\Gamma)$. Com efeito,

$$
\begin{aligned}
\left\|f-\sum_{m \in F} \lambda_{m}^{\varepsilon} \theta_{m}\right\|_{L^{2}(\Gamma)} & \leqq\left\|f-\sum_{m \in F} \lambda_{m}^{\varepsilon} \theta_{m}\right\|_{\infty}\left(\int_{\Gamma}|d s|\right)^{1 / 2}< \\
& <\frac{\varepsilon}{\left(\|\tilde{K}\|_{L^{2}(\Gamma)}+1\right)^{1 / 2}}
\end{aligned}
$$

Como por (III.2.1), temos

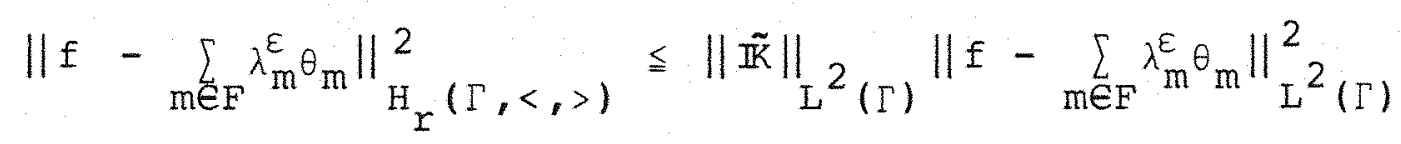

segue-se

$$
\| f-\sum_{m \in F} \lambda_{m_{m}^{\varepsilon} \|_{H_{r}}(\Gamma,<,>)}<\varepsilon
$$

Pela propriedade fundamental de conjuntos ortonormais (Ver [6], pag. 83, teorema 4.16) tem-se

$$
\| f-\sum_{m=-N}^{N}\left\langle\theta_{m}, f>\theta_{m}\left\|^{2} \leqq\right\| f-\sum_{m=-N}^{N} \lambda_{m}^{\varepsilon_{m}} \theta_{m} \|^{2}<\varepsilon^{2}\right.
$$

- qual nos diz que toda funcão $f \in H_{r}\left(\Gamma_{1}<,>\right)$ pode-se escrever da forma

$$
f=\sum_{m=-\infty}^{+\infty}<\theta_{m}, f>\theta_{m}
$$

Combinando isto com o Teorema da base (Ver [1], pag. 61, teorema 4.6) vemos que o conjunto ortonormal $\left\{\theta_{\mathrm{m}}\right\}$ é uma base ortonormal do espaco $\operatorname{Hr}(\Gamma,<,>)$. 
Note que (III.3.4) é a expansão em séries de Fourier da função f. Tambēm, observe que a expressão (III.1.13) não é a decomposição espectral do nücleo $\tilde{\mathrm{K}}$ em $\mathrm{H}_{\mathrm{r}}(\Gamma,<,>)$, pois $\zeta^{\mathrm{n}} L^{\mathrm{m}}$ no sentido de $\langle$,$\rangle em \Gamma$ mas não no sentido $L^{2}(\Gamma)$.

\section{Outra Base para o Espaço $H_{r}\left(\Gamma_{1}<,>\right)$}

Exibiremos uma base para o espaço $\mathrm{H}_{r}(\Gamma,<,>)$ formada pelas autofunções de um operador neste espaço definido usando o operador integral $\tilde{\mathbb{K}}$.

Primeiro, vamos definir este novo operador e calcular suas auto-funções.

Seja J o operador de conjugação. De (III.3.2) segue

$$
J \bar{K} z^{m}=-\left.i r^{2 \mid m+I}\right|_{z} ^{-(m+1)} e^{i \theta(z)}, m \in \mathbb{Z}
$$

de onde

$$
Z e^{-i \theta} J \tilde{\mathbb{K}} z^{m}=-\left.i r^{2 \mid m+1}\right|_{z} ^{-m}, m \in \mathbb{Z}
$$
Defina o operador $\tilde{I K}: H(\Gamma,<,>) \rightarrow H(\Gamma,<,>)$ por$$
\hat{\mathbb{K}}:=z e^{-i \theta} J \tilde{\mathbb{K}}
$$

De (III.4.1), vemos que o efeito deste operador sobre as funções $z^{m}$, mez, é dado por

(III.4.2)

$$
\mathbb{I} Z^{m}= \begin{cases}-i r^{2}|m+1|_{z^{-m}}, & m \in Z^{*} \\ -i r^{2} \cdot 1 & , m=0\end{cases}
$$


de onde segue

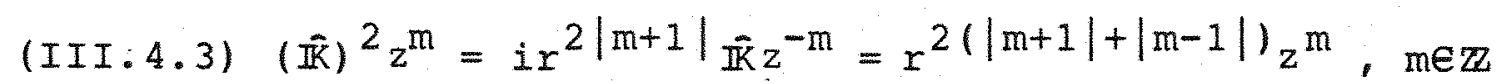

Podemos concluir que os sub espaços gerados pelas po tências $z^{\mathrm{m}}$ e $z^{-\mathrm{m}}$ são invariantes por $\hat{\mathbb{K}}$, portanto as autofunções deste operador serão combinações lineares delas.

Descreveremos as autofunções de $\widehat{\mathbb{K}}$ (não esquecer o fá to de que $\overline{\mathbb{K}}$ é um operador antilinear).

Com efeito, de (III.4.2) segue

(III. 4.4) $\overline{\mathbb{K}}\left(r^{-1} z^{m} \pm r z^{-m}\right)=-i r^{2 m}\left(r z^{-m} \pm r^{-1} z^{m}\right)$

$$
= \pm i r^{2 m}\left(r^{-1} z^{m} \pm r z^{-m}\right), m \geqq 1
$$

e

$$
\hat{\mathbb{K}} \cdot 1=-i r^{2} \cdot 1, m=0
$$

Logo $\left\{1,\left[r^{-1} z^{m} \pm r z^{-m}\right]\right\}_{m=1}^{+\infty}$ é o conjunto de todas as autofunções de $\hat{\mathbb{K}}$.

Agora, vamos mostrar que o conjunto $\left\{1,\left[r^{-1} z^{m} \pm r z^{-m}\right]\right\}_{m=1}^{+\infty}$ é ortonormal em $\langle$,$\rangle . Em primeiro 1 u-$ gar, achemos o efeito do operador $\tilde{\mathbb{K}}$ sobre este conjunto.

Pela definição de $\hat{\mathbb{K}}$, temos $\tilde{\mathbb{K}}=J e^{i \theta} z^{-1} \hat{\mathbb{K}}$. De (III.4.4), segue 
(III.4.5) $\tilde{\mathbb{K}}\left(r^{-1} z^{m} \pm r z^{-m}\right)=J e^{i \theta} z^{-1} \tilde{\mathbb{K}}\left(r^{-1} z^{m} \pm r z^{-m}\right)$

$$
=\frac{i r^{2 m} e^{-i \theta}}{\bar{z}}\left(r^{-1-m} \pm r \bar{z}^{-m}\right), m \geqq 1
$$

e

$$
\begin{aligned}
\tilde{\mathbb{K}} \cdot I & =\mathrm{Je}^{i \theta} z^{-1} \overline{\mathbb{K}} \cdot I \\
& =\frac{i r^{2} e^{-i \theta}}{\bar{z}}, m=0
\end{aligned}
$$

(III.4.6)

Definamos as funções $A_{m}^{ \pm}, m \in \mathbb{Z}^{+} U\{0\}$ por

$$
\begin{aligned}
A_{m}^{ \pm} & =r^{-1} z^{m} \pm r z^{-m}, & m \geq 1 \\
& =1 & , m=0
\end{aligned}
$$

Temos o seguinte resultado

Lema III. 4.1

$$
\begin{aligned}
& \left\langle A_{m}^{ \pm}, A_{n}^{ \pm}\right\rangle=\left\|A_{m}^{ \pm}\right\| \delta_{m, n}, \text { onde } \\
& \left\|A_{m}^{ \pm}\right\|= \begin{cases}2 \sqrt{\pi} r^{m}, & m=n, m \geqq 1 \\
\sqrt{2 \pi r}, & m=n=0\end{cases}
\end{aligned}
$$

Prova - Seja $m \neq n, m, n>0$. De (III.4.5)

$$
\begin{aligned}
\left\langle\mathrm{A}_{\mathrm{m}}^{ \pm}, \mathrm{A}_{\mathrm{n}}^{ \pm}\right\rangle & =\left(\mathrm{A}_{\mathrm{m}^{\prime}}^{ \pm}, \tilde{\mathrm{K}} \mathrm{A}_{\mathrm{n}}^{ \pm}\right) \\
& = \pm i r^{2 m} \int_{\Gamma}\left(r^{-1-m} \pm r^{-m}\right)\left(r^{-1-n} \pm r \bar{z}^{-n}\right) \frac{d \bar{z}}{\bar{z}} \\
& = \pm i r^{2 m} \int_{\Gamma}^{\left(r^{-1} z^{m} \pm r z^{-m}\right)\left(r^{-1} z^{n} \pm r z^{-n}\right) \frac{d z}{z}} \\
& = \pm i r^{2 m} \int_{\Gamma}^{\left(x^{-2} z^{m+n}+r^{2} z^{-(m+n)} \pm z^{-m+n} \pm z^{m-n}\right) \frac{d z}{z}}
\end{aligned}
$$


Se $m+n>0$ as integrais $\int_{\Gamma} z^{m+n} \frac{d z}{z}$ e $\int_{\Gamma} z^{-(m+n)} \frac{d z}{z}$ são nulas.

Por outro lado, como $m \neq n$ e positivos, temos

$$
\int_{\Gamma} z^{-m+n} \frac{d z}{z}=\int_{\Gamma} z^{m-n} \frac{d z}{z}=0
$$

\section{Então}

$$
\left\langle A_{m}^{ \pm}, A_{n}^{ \pm}\right\rangle=0 \quad \forall m, n \in \mathbb{N}, m \neq n
$$

No caso $m \geqq 1$ e $n=0$, de (III.4.6) tem-se

$$
\begin{aligned}
\left\langle\mathrm{A}_{\mathrm{m}}^{ \pm} \mathrm{A}_{\mathrm{O}}\right\rangle & =\left(\mathrm{A}_{\mathrm{m}}^{ \pm}, \tilde{\mathrm{K}} \mathrm{A}_{0}\right) \\
& =\int_{\Gamma}\left(r^{-1} \bar{z}^{\mathrm{m}} \pm r^{-m}\right) \frac{i r^{2} e^{-i \theta}}{\bar{z}} d s \\
& =i r^{2} \frac{\int_{\Gamma}\left(r^{-1} z^{m} \pm r z^{-m}\right) \frac{d z}{z}}{d s}=0
\end{aligned}
$$

No caso $\mathrm{m}, \mathrm{n}>0 ; \mathrm{n}=\mathrm{m}$

$$
\begin{aligned}
\left\langle A_{m}^{ \pm}, A_{m}^{ \pm}\right\rangle & =\left(A_{m}^{ \pm}, \tilde{K} A_{m}^{ \pm}\right) \\
& =\int_{\Gamma}\left(r^{-1} \bar{z}^{m} \pm r^{-m}\right)\left(\frac{ \pm i r^{2 m}}{\bar{z}}\left(r^{-1} \bar{z}^{m} \pm r^{-m}\right)\right) d \bar{z} \\
& = \pm i r^{2 m} \int_{\Gamma} \bar{z}^{-1}\left(r^{-1-m} \bar{z}^{m} \pm r \bar{z}^{-m}\right)^{2} d \bar{z} \\
& = \pm i r^{2 m} \int_{\Gamma}\left(r^{-2-2 m-1} \pm 2+r^{\left.2-\bar{z}^{-2 m-1}\right) d \bar{z}}\right. \\
& = \pm 2 i r^{2 m} \frac{d z}{z}
\end{aligned}
$$

pois, $\int_{\Gamma} z^{2 m-1} d z=\int_{\Gamma} z^{-2 m-1} d z=0$ para $m \geqq 1$.

Iogo

$$
\left\langle\mathrm{A}_{\mathrm{m}}^{+}, \mathrm{A}_{\mathrm{m}}^{+}\right\rangle=4 \pi \mathrm{r}^{2 \mathrm{~m}}
$$


Por outro lado,

$$
\begin{aligned}
& \left\langle\mathrm{A}_{\mathrm{m}^{\prime}}^{+}, \mathrm{A}_{\mathrm{m}}^{-}\right\rangle=\left(\mathrm{A}_{\mathrm{m}^{\prime}}^{+}, \tilde{K} \mathrm{~A}_{\mathrm{m}^{-}}^{-}\right) \\
& =\int_{\Gamma}\left(r^{-1-m}+r \bar{z}^{-m}\right)\left(-\frac{i r^{2 m}}{\bar{z}}\left(r^{-1} \bar{z}^{-m}-r \bar{z}^{-m}\right) e^{-i \theta}\right) d s \\
& =-i r^{2 m} \overline{\int_{\Gamma}\left(r^{-2} z^{2 m}-r^{2} z^{-2 m}\right) \frac{d z}{z}} \\
& =0
\end{aligned}
$$

Se $m=0$, temos

$$
\begin{aligned}
\left\langle A_{0}, A_{0}\right\rangle & =\left(A_{0}, \overline{\mathbb{K}} A_{0}\right) \\
& =\int_{\Gamma} \frac{i r^{2} e^{-i \theta}}{\bar{z}} d s \\
& =i r^{2} \frac{\int_{\Gamma} \frac{d z}{z}}{\therefore} \\
& =2 \pi r^{2}
\end{aligned}
$$

Dai, obtemos

$$
\left\|A_{m}^{ \pm}\right\|= \begin{cases}2 \sqrt{\pi} x^{m}, & m \geqq 1 \\ \sqrt{2 \pi} r, & m=0\end{cases}
$$

Portanto

$$
\left\langle\mathrm{A}_{\mathrm{m}}^{ \pm}, \mathrm{A}_{\mathrm{n}}^{ \pm}\right\rangle=\left\|\mathrm{A}_{\mathrm{m}}^{ \pm}\right\|^{2} \delta_{\mathrm{m}, \mathrm{n}}
$$

Como queriamos demonstrar.

Segue-se do lema anterior que o conjunto

$\left\{\frac{1}{\sqrt{2 \pi} r}, \frac{1}{2 \sqrt{\pi} r^{m}}\left[r^{-1} z^{m} \pm r z^{-m}\right]\right\}_{m=1}^{+\infty}$ é ortonormal em $H_{r}(\Gamma,<,>)$. 
Ele é uma base ortonormal de $H_{r}(\Gamma,\langle\rangle),$,$\quad pois gera a base$ $\left\{\theta_{\mathrm{m}}\right\}$.

Note que, quando $r \rightarrow 1$ obtemos o conjunto

$$
\left\{\frac{1}{\sqrt{2 \pi}}, \frac{1}{\sqrt{\pi}} \cos m \theta, \frac{1}{\sqrt{\pi}} \operatorname{sen} m \theta\right\}_{m=1}^{+\infty}
$$

ortonormal em $\mathrm{L}^{2}\left(\mathrm{~S}^{1}\right)$, como na teoría clässica.

Faremos mais simples a notação, escrevendo

$$
\alpha_{m}^{ \pm}=\frac{1}{2 \sqrt{\pi} r^{m}}\left[r^{-1} z^{m} \pm r z^{-m}\right], m \geqq 1
$$

e

$$
\alpha_{0}=\frac{1}{\sqrt{2 \pi r}}
$$

\section{Observação}

Uma primeirá observação é que o operador $e^{i \theta} \tilde{\mathbb{K}}$ aplica funções analiticas em funções anti-analiticas.

$$
\text { Com efeito, de (III.4.5) e (III.4.6) segue }
$$

(III.4.7)

$$
\left(e^{i \theta} \tilde{\mathbb{K}}\right) \cdot 1=\frac{i r^{2} \cdot 1}{\bar{z}}
$$

e

(III.4.8)

$$
\left(e^{i \theta} \tilde{\mathbb{K}}\right) \cdot\left[r^{-1} z^{m} \pm r z^{-m}\right]= \pm \frac{i r^{2 m}}{\bar{z}}\left[r^{-1-m} \pm r \bar{z}^{-m}\right]
$$

de onde,

$$
\left[e^{i \theta} \tilde{\mathbb{K}}\right]\left(\alpha_{m}^{ \pm}\right)= \pm \frac{i}{\bar{z}} r^{2 m}-\frac{ \pm}{m}, m \geqq 1
$$

e

$$
\left[e^{i \theta} \tilde{\mathbb{K}}\right]\left(\alpha_{0}\right)=\frac{i}{\bar{z}} r^{2} \alpha_{0}
$$


Então a representação do operador linear $e^{i \theta} \tilde{I K}$ do espaço das funções analiticas com base $\left(\alpha_{0}, \alpha_{m}^{ \pm}\right)_{m=1}^{+\infty} \mathrm{mp}$ espaço das funções anti-analiticas com base $\left(\alpha_{0}, \bar{\alpha}_{m}^{\infty}\right)_{m=1}^{+\infty}$ é dada pela matriz

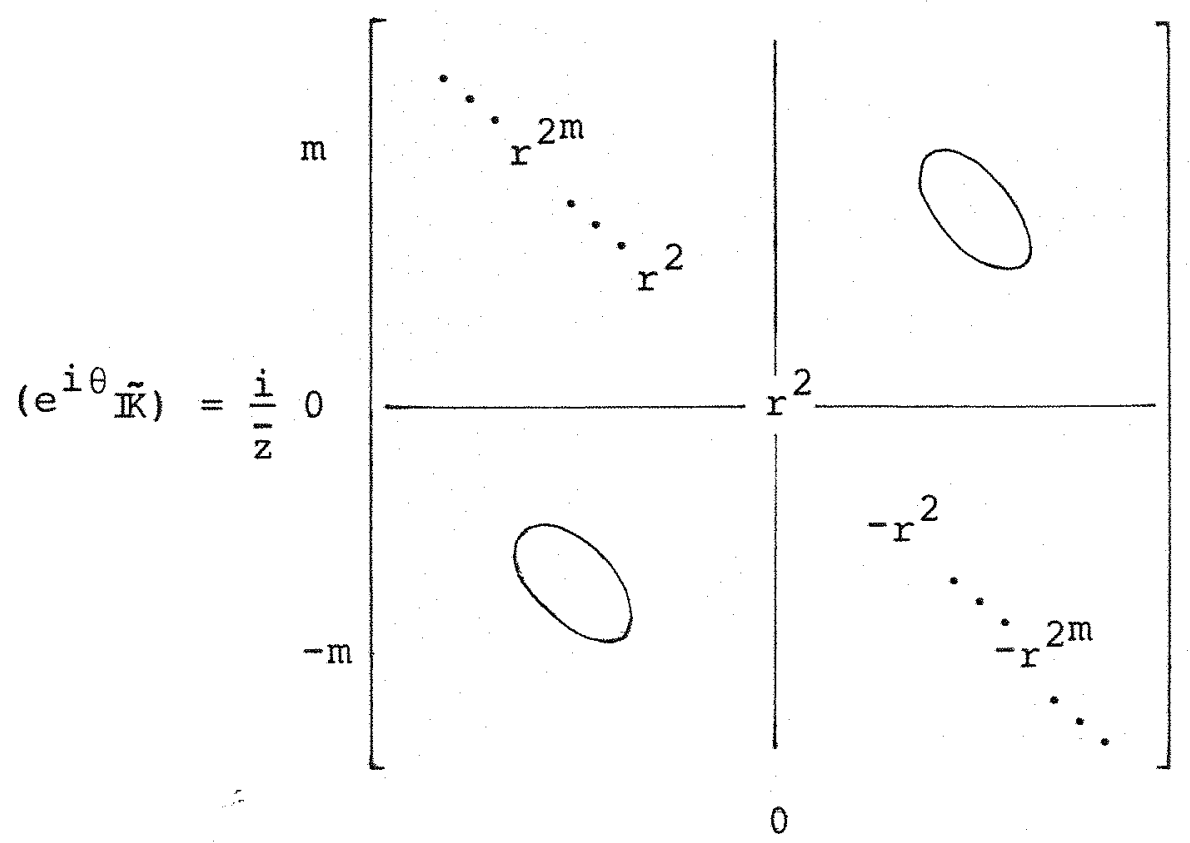

onde $0<r<1$. Quando $r \rightarrow 1$, obtemos

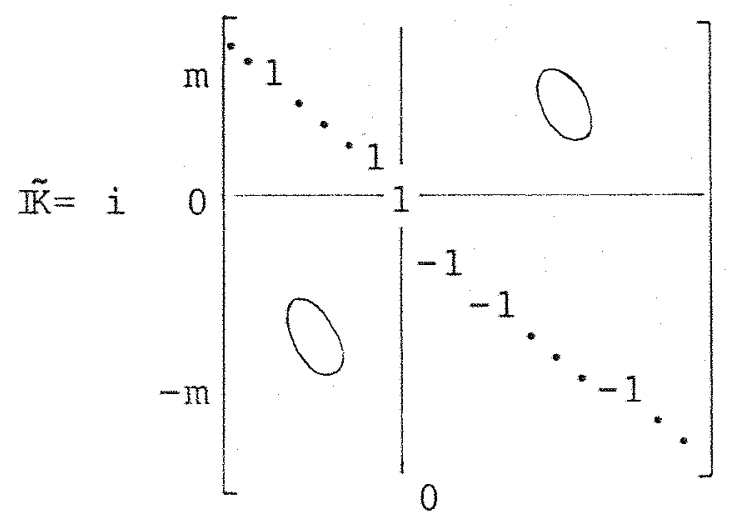




\section{Caracterizações do Completamento de $H$}

Por Mergelyan, dada $f \in C(\Gamma)$ existe uma sequência

$\left\{R_{N}=\sum_{n=-N}^{N} a_{n}^{N} z^{n}\right\}$ de funções racionais com polos em $0 \in \Omega$ (no interior de $\Gamma$ l e em que converge uniformemente para $f$ em $\Gamma$. Usando a fórmula integral de Cauchy, pelo dito acima segue

$$
a_{m}=\frac{1}{2 \pi i} \int_{\Gamma} \frac{R_{N}}{z^{m+1}} d z \underset{N \rightarrow+\infty}{\longrightarrow} \frac{1}{2 \pi i} \int_{\Gamma} \frac{f}{z^{m+1}} d z:=a_{m}
$$

Por definição a série de Laurent formal de $f$ é

$$
\pm \sim \sum_{n=-\infty}^{+\infty} a_{n} z^{n}
$$

Esta série é puramente formal. Mas dada a curva $\Gamma$ e escolhidas a origem no seu interior $\Omega$ e o disco $D(0 ; r) \subset \Omega$, $0<r<1$, mostraremos que a série converge no sentido de $\mathrm{H}_{\mathrm{r}}(\Gamma,<,>)$. Observe primeiramente, que valem as inclusões continuas

$$
C(\Gamma) \subset L^{2}(\Gamma) \subset \hat{\mathrm{H}}_{\Gamma}(\Gamma,<,>)
$$

isto vem de (III.2.1). De onde $R_{N} \rightarrow f$ em $L^{2}(\Gamma)$ e portanto $R_{N} \rightarrow f e m \hat{\mathrm{H}}_{I}(\Gamma,<,>)$

$$
\text { Em particular }\left\langle R_{N}, \theta_{m}>\underset{N \rightarrow+\infty}{\rightarrow}, f_{m}>\right.\text {, mas }
$$




$$
\begin{aligned}
& <R_{N}, \theta_{m}>=\left(R_{N}, \tilde{K} \theta_{m}\right)=i r|m+1|+|m| \int_{\Gamma} \bar{R}_{N} \bar{\theta}_{-(m+1)} d \bar{z} \\
& =\frac{i r|m+1|}{\sqrt{2 \pi}} \int_{\Gamma} R_{N} z^{-(m+1)} d z \\
& =\frac{i r|m+1|}{\sqrt{2 \pi}}(-2 \pi i) a_{m}^{N} \underset{N \rightarrow+\infty}{\longrightarrow} \sqrt{2 \pi} r|m+1| \overline{a_{m}}
\end{aligned}
$$

pelo resultado anterior.

\section{Logo}

(III.5.1) $\left.\quad f, \theta_{m}\right\rangle=\sqrt{2 \pi} r|m+1| \bar{a}_{m}$ e $\left\langle\theta_{m}, f>\theta_{m}=a_{m} z^{m}\right.$

portanto

(III.5.2)

$$
E=\sum_{m=-\infty}^{+\infty} a_{m} z^{m} \text { em } \hat{H}_{r}(\Gamma,<,>)
$$

Dai, segue o resultado

Teorema III.5.1 - A sërie formal de Laurent de $f \in C(\Gamma)$ con verge a $f$ no sentido de $\hat{\mathrm{H}}_{r}(\Gamma,<,>)$.

Note que nossa construção permite definir série de Laurent não só para $€ \in C(\Gamma)$ mas para todo elemento de $\overline{\mathrm{H}}_{Y}(\Gamma,<,>)$.

$$
\text { Agora, considere } f \in C(\Gamma) \text {. Seja a }>0 \text { oraio }
$$

do maior disco aberto contido em $\Omega$ e b $>0$ o raio do menor disco contendo $\Gamma$.

Teorema III. 5.2 - Para todo $x<\min \left(a, \frac{1}{b}\right)$, tem-se

$$
\sum_{n=-\infty}^{+\infty} r^{2|n+1|}\left|a_{n}\right|^{2}<+\infty
$$


Prova - Usar a identidade de Parseval em relação à base ortonormal $\left\{\theta_{n}\right\}$ combinado com (III.5.1)

$$
\text { No caso especial } \Gamma=s^{1} \text { e } r \rightarrow 1 \text {, temos que }
$$

$$
f \sim \sum_{n=-\infty}^{+\infty} a_{n} z^{n}
$$

é a sërie de Fourier de $f \in C\left(S^{1}\right) \operatorname{com} \sum_{n=-\infty}^{+\infty}\left|a_{n}\right|^{2}<+\infty$. $\log 0 L^{2}\left(S^{1}\right)=\bar{H}_{1}\left(S^{1},<,>\right)$. 
Outra caracterização de $\mathrm{H}_{r}\left(\Gamma, \Gamma_{,}>\right)$

Dada a curva $\Gamma$ e escolhidas a origem no seu inte rior $\Omega$ e o disco $\mathrm{D}(0 ; r) \subset \Omega, 0<r<1$, considere a função $h \in \hat{H}_{r}(\Gamma,<,>)$ com série

(III. 5.4)

$$
h=\sum_{m=0}^{+\infty} a_{m} z^{m}
$$

Tomando $|z|=\rho<r$, pela desigualdade de Cauchy-Schwarz em $H_{r}(\Gamma,<,>)$ e de (III.5.3) tem-se

$$
\begin{aligned}
& \sum_{m=0}^{+\infty}\left|a_{m}\right| \rho^{m}=\sum_{m=0}^{+\infty}\left(r^{m}\left|a_{m}\right|\left(\frac{\rho}{r}\right)^{m}\right) \leqq \\
& \leqq\left(\sum_{m=0}^{+\infty} r^{2 m}\left|a_{m}\right|^{2}\right)^{1 / 2}\left(\sum_{n=0}^{+\infty}\left(\frac{\rho}{r}\right)^{2 m}\right)^{1 / 2}<+\infty
\end{aligned}
$$

Logo, o raio de convergência $R_{i}$ da série (III.5.4), satisfaz $R_{i} \geqq r$.

Analogamente, dada uma função $g \in \overline{\mathrm{H}}_{\Gamma}(\Gamma,<,>)$ com sé-

rie

(III:5.5)

$$
g=\sum_{m=1}^{+\infty} a_{-m} z^{-m}
$$

temos que se $|z|=\rho_{1}>r^{-1}$; da desigualdade de Cauchy-Schwarz em $H_{r}(\Gamma,<,>)$ e de (III.5.3) segue

$$
\begin{aligned}
& \sum_{m=1}^{+\infty}\left|a_{-m}\right| \rho_{1}^{-m}=\sum_{m=1}^{+\infty}\left(r^{m}\left|a_{-m}\right|\right)\left(r \rho_{1}\right)^{-m} \\
& \leqq\left(\sum_{m=1}^{+\infty} r^{2 m}\left|a_{-m}\right|^{2}\right)^{1 / 2}\left(\sum_{m=1}^{+\infty}\left(r \rho_{1}\right)^{-2 m}\right)^{1 / 2}<+\infty
\end{aligned}
$$


De onde, o raio de convergēncia $R_{e}$ da série (III.5.5) satisfaz $R_{e} \leqq r^{-1}$.

Em conseqüēncia,

$$
\hat{\mathrm{H}}_{\mathrm{I}}\left(\Gamma, r_{,}\right) \subset A(D(0 ; r)) \cap A\left(D\left(\infty ; r^{-1}\right)\right)
$$

Nosso objetivo agora é caracterizar o espaço $\overline{\mathrm{H}}_{\Gamma}(\Gamma,<,>)$ no sentido das normas $L^{2}\left(S_{r}\right)$ e $L^{2}\left(S_{r-1}\right)$.

$$
\begin{array}{ll}
\text { Seja } & f(z)=\sum_{n=-\infty}^{+\infty} a_{n} z^{n} \in \hat{H}_{r}(\Gamma,<,>), \text { temos } \\
w_{f}^{i}(z)=\sum_{n=0}^{+\infty} a_{n} z^{n} \text { e } w_{f}^{e}(z)=\sum_{n=1}^{+\infty} a_{-n} z^{-n}
\end{array}
$$

com

$$
\begin{aligned}
& \left\|w_{f}^{i}\right\|_{L^{2}\left(s_{I}\right)}^{2}=\sum_{n=0}^{+\infty} r^{2 n}\left|a_{n}\right|^{2}<+\infty \\
& \left\|w_{f}^{e}\right\|_{L^{2}\left(s_{r-1}\right)}^{2}=\sum_{n=1}^{+\infty} r^{-2 n}\left|a_{-n}\right|^{2}<+\infty
\end{aligned}
$$

Se $0<\rho<r$, defina

$$
w_{f, \rho}^{i}(z):=w_{r}^{i}\left(\frac{\rho}{r} z\right)=\sum_{n=0}^{+\infty} a_{n}\left(\frac{E \rho}{r}\right)^{n} z^{n} \in L^{2}\left(S_{r}\right)
$$

segue de $\left|\frac{p}{r} a_{n}\right|<\left|a_{n}\right|$ combinado com (III.5.6)

Mostraremos que $\left\|w_{f}^{i}-w_{f}^{i},\right\|_{L}^{2}\left(S_{L}\right) \rightarrow 0$ quando $\rho \rightarrow r$ 
Pela identidade de Parseval em $\mathrm{L}^{2}\left(\mathrm{~S}_{\mathrm{r}}\right)$, tem-se

$$
\begin{aligned}
\left\|w_{r}^{i}-w_{E, \rho}^{i}\right\|_{L^{2}\left(s_{r}\right)}^{2} & =\left\|\sum_{n=0}^{+\infty} a_{n} z^{n}-\sum_{n=0}^{+\infty} a_{n}\left(\frac{\rho}{r}\right)^{n} z^{n}\right\|_{L^{2}}^{2}\left(s_{r}\right) \\
& =\left\|\sum_{n=0}^{+\infty} a_{n}\left[1-\left(\frac{\rho}{r}\right)^{n}\right] z^{n}\right\|_{L^{2}\left(s_{r}\right)}^{2} \\
& =2 \pi \sum_{n=0}^{+\infty}\left|a_{n}\right|^{2}{ }^{2 n}\left[1-\left(\frac{\rho}{r}\right)^{n}\right]^{2}
\end{aligned}
$$

Por (III.5.6), dado $\varepsilon>0$ existe $N \in \mathbb{N}$ tal que

(III.5.8)

$$
2 \pi \sum_{n=N+1}^{+\infty}\left|a_{n}\right|^{2} r^{2 n}<\frac{\varepsilon^{2}}{2}
$$

$$
\text { Note que se } N \geqq n \text {, tem-se }\left[1-\left(\frac{\rho}{r}\right)^{n}\right]<\left[1-\left(\frac{\rho}{r}\right)^{N}\right]
$$

então

$$
\begin{aligned}
2 \pi \sum_{n=0}^{N}\left|a_{n}\right|^{2} r^{2 n}\left[1-\left(\frac{\rho}{r}\right)^{n}\right]^{2} & <2 \pi\left[1-\left(\frac{\rho}{r}\right)^{N}\right]^{2} \sum_{n=0}^{N}\left|a_{n}\right|^{2} r^{2 n} \leqq \\
& \leqq 2 \pi M\left[1-\left(\frac{\rho}{r}\right)^{N}\right]^{2}
\end{aligned}
$$

onde $M$ é soma da série em (III.5.6).

$$
\text { Como } \operatorname{Lim}_{\rho \rightarrow r}\left[1-\left(\frac{\rho}{r}\right)^{N}\right]^{2}=0 \text {, dado } \varepsilon>0 \exists 0<\rho_{0}<r ;
$$

$\rho_{0}<\rho<r$ tal que

$$
1-\left(\frac{\rho_{O}}{r}\right)^{N}<\frac{\varepsilon}{\sqrt{4 \pi M}}
$$

Então, 
(III.5.9)

$$
2 \pi \sum_{n=0}^{N}\left|a_{n}\right|^{2} x^{2 n}\left[1-\left(\frac{\rho}{x}\right)^{n}\right]^{2}<\frac{\varepsilon^{2}}{2}
$$

Segue-se, de (III.5.8) e (III.5.9) que dado

$\varepsilon>0 \exists 0<\rho_{0}<r$ tal que $\rho_{0}<\rho<r$ implica

$$
\left\|w_{f}^{i}-w_{f, \rho}^{i}\right\|_{L^{2}\left(S_{r}\right)}^{2}<\varepsilon^{2}
$$

Analogamente, se $0<\rho<r$ defina

$$
w_{f, \rho}^{e}(z):=w_{f}^{e}\left(\frac{r}{\rho} z\right)=\sum_{n=1}^{+\infty} a_{-n}\left(\frac{\rho}{r}\right) n_{z}-n
$$

Note que $w_{f, \rho}^{e} \in L^{2}\left(s_{r}-1\right)$

Mostraremos que $\left\|w_{f}^{e}-w_{f, p}^{e}\right\|_{L^{2}\left(S_{r^{-1}}\right)}^{2} \rightarrow 0$ quando $\rho \rightarrow r$

Usando a identidade de Parseval em $L^{2}\left(s_{r^{-1}}\right)$, tem-se

$$
\begin{aligned}
& \left.\left\|w_{f}^{e}(z)-w_{f, \rho}^{e}(z)\right\|_{L^{2}\left(S_{r^{-1}}\right)}^{2}=\left\|\sum_{n=1}^{+\infty} a_{-n} z^{-n}-\sum_{n=1}^{+\infty} a_{-n}\left(\frac{\rho}{r}\right)^{n} z^{-n}\right\|_{L^{2}\left(s_{L}-1\right.}^{2}\right) \\
& \left.=\left\|\sum_{n=1}^{+\infty}\left[1-\left(\frac{\rho}{r}\right)^{n}\right] a_{-n^{z}}{ }^{-n}\right\|_{L^{2}}^{2} s_{r-1}\right) \\
& =2 \pi \sum_{n=1}^{+\infty}\left[1-\left(\frac{p}{r}\right)^{n}\right]^{2}\left|a_{-n}\right|^{2} x^{-2 n}
\end{aligned}
$$

Por (III.5.7), dado $\varepsilon_{1}>0$ existe $N_{1} \in \mathbb{N}$ tal que

(III.5.10)

$$
2 \pi \sum_{n=N_{1}+1}^{+\infty}\left|a_{-n}\right|^{2} r^{-2 n}<\frac{\varepsilon_{1}^{2}}{2}
$$


Por outro lado, pelo mesmo raciocínio feito na demonstração anterior tem-se

$$
\begin{aligned}
2 \pi \sum_{n=1}^{N}\left[1-\left(\frac{\rho}{r}\right)^{n}\right]^{2}\left|a_{-n}\right|^{2} r^{-2 n} & <2 \pi\left[1-\left(\frac{\rho}{r}\right)^{N_{1}}\right] \sum_{n=1}^{N}\left|a_{-n}\right|^{2} r^{-2 n} \leqq \\
& \leqq 2 \pi M_{1}\left[1-\left(\frac{\rho}{r}\right)^{N_{1}}\right]^{2}
\end{aligned}
$$

onde $M_{1}$ é a série em (III.5.7)

$$
\begin{aligned}
\text { Como }\left[1-\left(\frac{\rho}{r}\right)^{N_{1}}\right]^{2} \rightarrow 0 \text { quando } \rho \rightarrow r \text {, temos para } \\
\varepsilon_{1}>0 \text { existe } 0>\rho_{0}^{\prime}<r ; \rho_{0}^{\prime}<\rho<r e \\
\\
1-\left(\frac{\rho O}{r}\right)^{N_{1}}<\frac{\varepsilon_{1}}{\sqrt{4 \pi M_{1}}}
\end{aligned}
$$

Então,

(III.5.11)

$$
2 \pi \sum_{n=1}^{N}\left|a_{-n}\right| r^{-2 n}\left[1-\left(\frac{\rho}{r}\right)^{n}\right]^{2}<\frac{\varepsilon_{1}^{2}}{2}
$$

Segue-se de (III.5.10) e (III.5.11) que dado $\varepsilon_{1}>0 \exists \quad 0<\rho_{0}^{\prime}<r$ tal que $\rho_{0}^{\prime}<\rho<r$ implica

$$
\left\|w_{f}^{e}-w_{f, \rho}^{e}\right\|_{L^{2}\left(S_{I^{-1}}\right)}^{2}<\varepsilon_{1}^{2}
$$

Observe que dada uma função $f \in H_{r}(\Gamma,<,>)$ a função $w_{f}^{i}$ além de ser analitica no disco $D(0 ; r)$ ela é o limite de Euncões analiticas no disco $D(0 ; \rho)$, com $\rho<r$, no sentido de $L^{2}\left(S_{r}\right)$ quando $\rho \rightarrow r$. Analogamente, $w_{f}^{e}$ é analitica no disco $D\left(0 ; x^{-1}\right)$ e é o limite de funções analiticas no disco 
$D\left(0 ; \rho^{\prime-1}\right), \rho^{\prime}<r$, no sentido de $L^{2}\left(S_{r-1}\right)$ quando $\rho^{\prime} \rightarrow r$.

Denotemos por

$$
\pi^{+}=A(D(0 ; r)) \cap L^{2}\left(S_{x}\right)^{+}
$$

e

$$
\Pi^{-}=A\left(D\left(\infty ; r^{-1}\right)\right) \cap L^{2}\left(S_{r^{-1}}\right)^{-}
$$

onde as intersecções são no sentido do parágrafo anterior e $L^{2}\left(S_{r}\right)^{+}=L^{2}\left(S_{r}\right) \cap_{n \geq 0}$ e $L^{2}\left(S_{r-1}\right)^{-}=L^{2}\left(S_{r-1}\right) \bigcap_{n<0}$.

Portanto

$$
\overline{\mathrm{H}}_{x}\left(\Gamma,\langle,>) \subset \pi^{+} \cap \pi^{-}\right.
$$

Na verdade, "temos uma igualdade em (III.5.12). Com efeito, seja $f \in \pi^{+} \cap \pi^{-}, f=g+h ; g \in \pi^{+}$e $h \in \pi^{-}$. Se $g \in \pi^{+}$, temos

$$
g=\sum_{n=0}^{+\infty} a_{n} z^{n}=\sum_{n=0}^{+\infty} a_{n}(\sqrt{2 \pi r}(n+1)) \theta_{n}
$$

$$
\text { Seja } 0<\rho<r \text { e defina }
$$

(III.5.14)

$$
g_{\rho}(z)=\sum_{n=0}^{+\infty}\left(\sqrt{2 \pi} a_{n} r^{n+1}\right)\left(\frac{\rho}{r}\right)^{n} \theta_{n}
$$

De (III.1.1), pela proposição (III.1.1) temos

$$
\left\|g-g_{p}\right\|_{H_{r}}^{2}\left(\Gamma_{,}<,>\right)=r\left\|g-g_{p}\right\|_{L}^{2}{ }^{2}\left(S_{r}\right)
$$


então, $g_{\rho} \frac{L^{2}\left(S_{r}\right)}{\rho \longrightarrow r} g$ implica $g_{\rho} \frac{H_{r}(\Gamma,<,>)}{\rho \rightarrow r} g$

Segue-se, usando a identidade de parseval em relação a base ortonormal $\left\{\theta_{n}\right\}$ combinado com o resultado de acima,

$$
\begin{gathered}
2 \pi \sum_{n=0}^{+\infty}\left(\frac{\rho}{r}\right)^{2 n}\left|a_{n}\right|^{2} r^{2(n+1)}=2 \pi \sum_{n=0}^{+\infty} \rho^{2 n} r^{2}\left|a_{n}\right|^{2} \frac{\rho}{\rho \longrightarrow r} \\
2 \pi \sum_{n=0}^{+\infty} r^{2(n+1)}\left|a_{n}\right|^{2}<+\infty
\end{gathered}
$$

Se $h \in \Pi^{-}$, temos

$$
h=\sum_{n=1}^{+\infty} a-n^{z^{-n}}=\sum_{n=1}^{+\infty} \sqrt{2 \pi} a_{-n^{r}} r_{-n}^{(1-n)}
$$

Para $0<\rho<r$, defina

$$
h_{\rho}(z)=\sum_{n=1}^{+\infty}\left(\sqrt{2 \pi} a_{-n} x^{1-n}\right)\left(\frac{\rho}{r}\right)^{n} \theta_{-n}
$$

De (III.1.1), pela proposição (III.1.1), temos

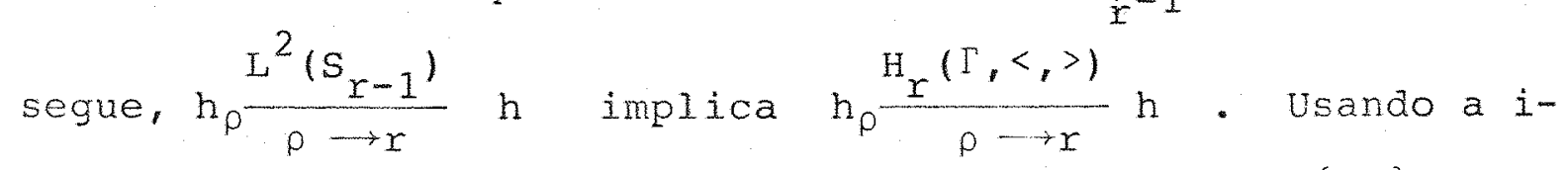
dentidade de parseval em relacão a base ortonormal $\left\{\theta_{n}\right\}$, obtemos 


$$
\begin{aligned}
2 \pi \sum_{n=1}^{+\infty}\left(\frac{\rho}{r}\right)^{2 n}\left|a_{-n}\right|^{2} r^{2(n-1)}=2 \pi \sum_{n=1}^{+\infty} 2 n_{r}-2\left|a_{-n}\right|^{2} \overrightarrow{\rho \rightarrow r} \\
\\
\longrightarrow 2 \pi \sum_{n=1}^{+\infty}\left|a_{-n}\right|^{2} r^{2(n-1)<+\infty}
\end{aligned}
$$

De (III.5.15) e (III.5.16), segue

$$
2 \pi \sum_{n=1}^{+\infty}\left|a_{n}\right|^{2} r^{2|n+1|<+\infty}
$$

Portanto $f \in \hat{\mathrm{H}}_{r}(\Gamma,<,>)$. De onde

$$
\hat{\mathrm{H}}_{Y}(\Gamma,<,>) \supset \Pi^{+} \cap \Pi^{-}
$$

Em conseqüència, temos o

Teorema (III.5.3) $\quad \hat{\mathrm{H}}_{\mathrm{r}}\left(\Gamma_{t}<,>\right)=\Pi^{+} \cap \mathrm{I}^{-}$

Note que, dada a condição

$$
\sum_{n=-\infty}^{+\infty} r^{2|n+1|}\left|a_{n}\right|^{2}<+\infty
$$

vamos ter uma familia infinita de curvas satisfazendo a condição
(III.5.17)
$D(0 ; r) \subset \Omega \subset D\left(0 ; r^{-1}\right)$ 


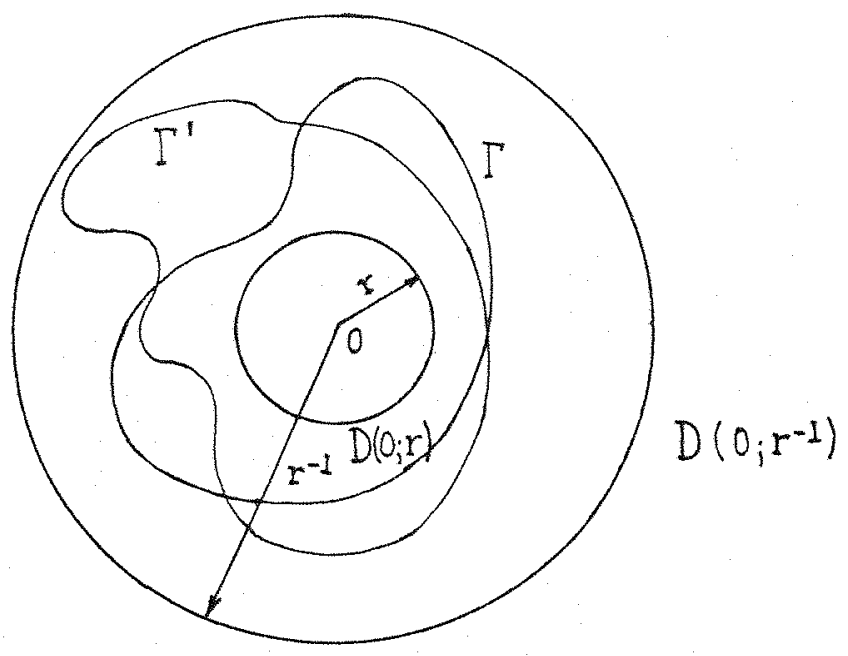

Proposicão (III.5.1) - Para quaisquer curvas $\Gamma$ e $\Gamma^{\prime}$ satisfazendo (III.5.17), tem-se

$$
\hat{\mathrm{H}}_{\Gamma}(\Gamma,<,>) \doteq \hat{\mathrm{H}}_{\Gamma}\left(\Gamma^{\prime},<,>\right)
$$

onde $\tilde{\approx}:=$ isomorfismo de espaços de Hilbert.

Prova - Considere duas curvas $\Gamma$ e $\Gamma^{\prime}$ que satisfazem a condição (III.5.17)

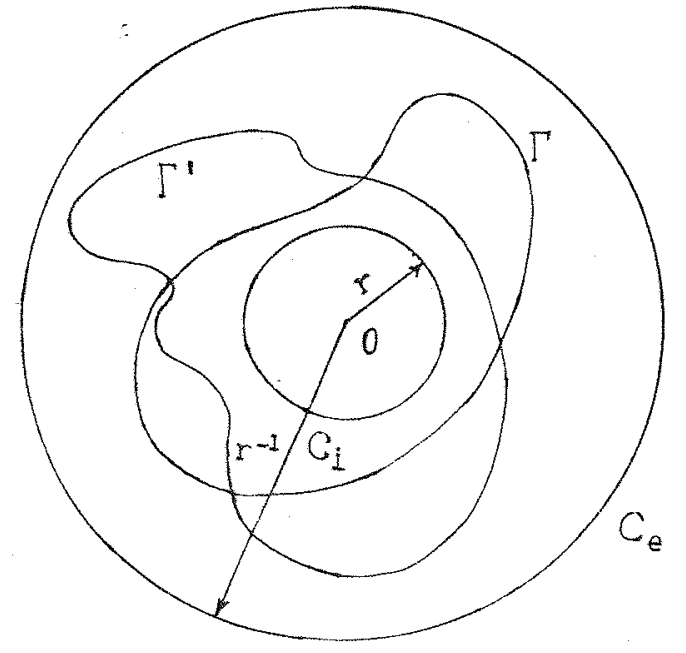

Seja $f \in R(\Gamma)=\{g: \Gamma \rightarrow \mathbb{I} ; g$ é uma função racional com polos em 0 e no $\infty\}$.

Temos que, conhecer $f \in R(T)$ determina conhecer f $E R\left(\Gamma^{\prime}\right)$ e como

$$
\|f\|_{\mathrm{H}_{\Gamma}(\Gamma,<,>)}=\|f\|_{\mathrm{H}_{\Gamma}(\Gamma,<,>1)}
$$


então, existe uma isometria $A$ de $R(\Gamma,<,>)$ sobre $\left.R\left(\Gamma^{\prime},<,\right\rangle^{\prime}\right)$. Sendo $R(\Gamma)$ e $R\left(\Gamma^{\prime}\right)$ denos em $C(\Gamma)$ e $C\left(\Gamma^{\prime}\right)$, respectivamente, podemos estender a isometria $A$ aos completamentos, i.e., existe um isomorfismo isométrico $\operatorname{deH}_{r}(\Gamma,<,>)$ em $H_{Y}\left(\Gamma^{\prime},<,>1\right)$. Logo está demonstrada a proposição.

\section{Construção de um Espaço de Hilbert de Funções Analiticas}

Em primeiro lugar, e conveniente fazer a seguinte observação: existem funções analíticas em $D(0 ; 1)$ definidas por

$$
f(z)=\sum_{n=0}^{+\infty} c_{n} z^{n},|z|<1
$$

que não podemos extender analiticamente a nenhum dominio contendo D $(0 ; 1)$ propriamente. Por exemplo, as séries "Lacunares" são do tipo (III.6.1) tal que a "maioria" dos coeficientes $c_{n}$ são nulos, isto ë, as séries "Lacunares" são da forma

$$
\sum_{k=1}^{\infty} a_{k}{ }^{n} k, \quad a_{k} \neq 0
$$

onde

$$
\lim _{k \rightarrow+\infty} \frac{k}{n_{k}}=0
$$

Esta ültima condição é suficiente para que a série não possua uma extensão analitica a nenhum aominio of $\supsetneq \mathrm{D}(0 ; 1)$. Este resul tado é estabelecido no Teorema do "gap" de Hadamard cujo enun- 
ciado é

Teorema (Ver [1], pag. 88, teorema II.7.1) - As séries (III.6.2) tem o circulo de convergência como fronteira natural sempre que exista um número fixo $\lambda>1$ tal que para todo $k \in \mathbb{N}$

$$
\frac{\mathrm{n}_{\mathrm{k}+1}}{\mathrm{n}_{\mathrm{k}}} \geqq \lambda
$$

Dada uma curva $\Gamma$ com $0 \in \Omega$ e $0<r<1$, construiremos um espaço de Hilbert de funções analiticas em $\mathbb{C} \backslash \Gamma$. Pelo Teorema (III.5.1)

$$
\hat{\mathrm{H}}_{r}(\mathrm{~T},<,>)=\Pi^{+} \cap \mathrm{I}^{-}
$$

Se $f \in \overline{\mathrm{H}}_{\Upsilon}(\Gamma,<,>)$, temos $\mathrm{w}_{f}^{i} \in \Pi^{+}$e $w_{f}^{e} \in \Pi^{-}$. Note que $w_{f}^{i} e$ we nem sempre possue extensöes analiticas para todo o dominio $\Omega$ e $\bar{\Omega}^{\mathrm{C}}$ respectivamente.

Consideremos o conjunto

$$
\bigcap_{z_{0} \in \Omega} \hat{H}\left(\Gamma, z_{0}, r_{z_{0}},<,>z_{0}^{\prime}\right.
$$

onde $\hat{H}\left(T, z_{0}, r_{z_{0}},\left\langle, z_{z_{0}}\right)=\Pi_{z_{0}}^{+} \cap \Pi_{z_{0}}^{-} \operatorname{com} \Pi_{z_{0}}^{+}=A\left(D\left(z_{0} ; r_{z_{0}}\right)\right) \cap L^{2}\left(S_{r_{z}}\right.\right.$ $e \Pi_{z_{0}}^{-}=A\left(D\left(z_{0} ; r_{z_{0}^{-1}}^{-1}\right) \cap L_{r_{0}}^{2}\left(s_{z_{0}}\right), 0<r_{z_{0}}<1\right.$.

Agora, aplique a transformacão conforme $z+\frac{1}{z}$ à curva $\Gamma$, como no desenho. 


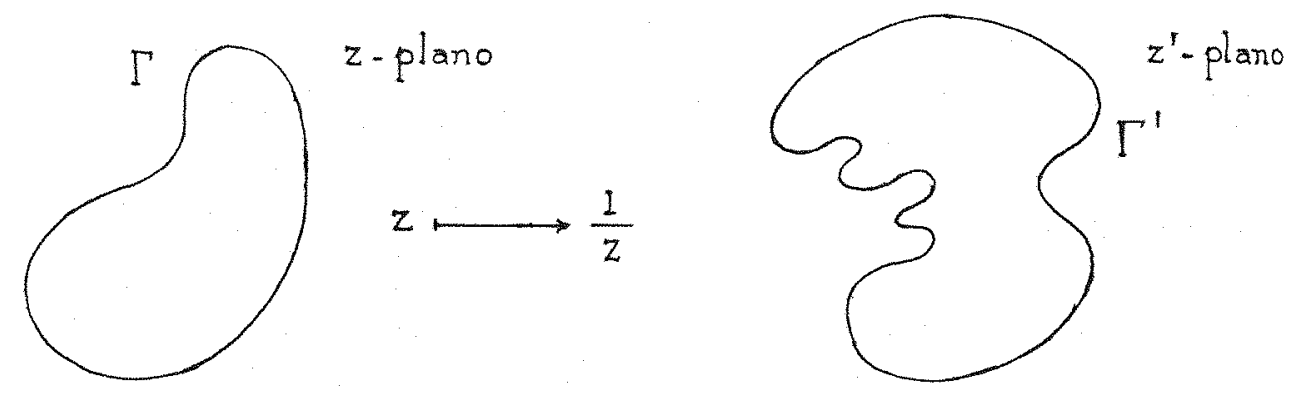

Então, todo z $\in \bar{\Omega}^{\mathrm{C}}$ é levado por esta transformação em $z^{\prime}=\frac{1}{z} \in \Omega^{\prime} \cdot$ Considere o conjunto

$$
\text { (III.6.3) } \bigcap_{z_{0}^{\prime} \in \Omega} \hat{H}\left(\Gamma^{\prime}, z_{0}^{\prime}, r_{z_{0}^{\prime}}^{\prime},<_{,}>z_{0}^{\prime}\right)
$$

onde os espaços $\hat{H}\left(\Gamma^{\prime}, z_{0}^{1}, r_{z_{0}^{\prime}}^{\prime}, r_{1} z_{z_{0}^{\prime}}\right)$ estão definidos de modo análogo aos espaços $\hat{H}\left(\Gamma, z_{0}, r_{z_{0}},<, z_{0}\right)$.

Por aplicação da transformação conforme $z^{\prime} \mapsto \frac{1}{z}$, , o conjunto definido em (III.6.3) é levado em

$(I I I: 6.4)$

$$
\bigcap_{z_{0}} \overline{\Omega^{\prime}} \bar{H}\left(\Gamma, z_{0}, r_{z_{0}}^{-1},<, z_{z_{0}}\right)
$$

Note que os produtos internos em (III.6.3) e (III.6.4) são os mesmos.

Dada a cobertura aberta $Q=\left\{D\left(z_{0} ; r_{z_{0}}\right)\right\}$ de $\Omega, e$ a cobertura aberta $Q^{\prime}=\left\{D\left(z_{0}^{\prime} ; r_{z_{0}^{\prime}}\right)\right\}_{z_{0}^{\prime} \in \Omega^{\prime}}$ de $\Omega^{\prime}$, pelo Teorema de Iindelöf, existem subcoberturas enumeráveis

$$
\Omega \subseteq \bigcup_{n=1}^{+\infty} D\left(z_{n} ; r_{n}\right) \text { e } \Omega^{\prime} \subseteq \bigcup_{m=1}^{+\infty} D\left(z_{m}^{\prime} ; r_{m}\right)
$$


Porêm, a imagem da cobertura enumerável $\bigcup_{m=1}^{+\infty} D\left(z_{m}^{\prime} ; r_{m}\right)$ de $\Omega^{\prime}$ pela aplicação $z^{\prime} \longrightarrow \frac{1}{z^{\prime}}=z$ é uma cobertura enumerável de $\bar{\Omega}^{C}$. Desde que a cada espaço $\hat{H}\left(\Gamma, z_{n}, r_{z_{n}},\langle,\rangle z_{n}\right)$ se associa um disco $D\left(z_{n} i r_{z_{n}}\right.$ ) lo mesmo acontece com os espaços

$H\left(\Gamma^{\prime}, z_{m}^{\prime}, r_{m},<, z_{m}, 1\right)$ obtemos o conjunto enumerável $\left\{\bar{H}_{n}\right\}_{n=1}^{+\infty}$ de espaços, onde $\hat{\mathrm{H}}_{\mathrm{n}}=\hat{\mathrm{H}}\left(\Gamma, \mathrm{z}_{\mathrm{n}}, r_{\mathrm{n}},\left\langle,{ }_{z_{n}}\right), \mathrm{z}_{\mathrm{n}} \in \Omega\right.$. Analogamente, obtemos o conjunto enumerável $\left\{\hat{\mathrm{H}}_{\mathrm{m}}\right\}_{\mathrm{m}=1}^{+\infty}$ de espaços, onde $\overline{\mathrm{H}}_{\mathrm{m}}=\mathrm{H}\left(\Gamma, \mathrm{z}_{\mathrm{m}}, \mathrm{r}_{\mathrm{m}}^{-1},\langle,\rangle_{z_{\mathrm{m}}}\right), \mathrm{z}_{\mathrm{m}} \in \bar{\Omega}^{\mathrm{C}}$.

\section{Tomando}

$$
\begin{gathered}
\Psi^{i}=\left\{g \in \hat{H}_{n=1}^{+\infty} \hat{H} ; g \equiv 0 \text { em } \bar{\Omega}^{c}\right\} \\
\Psi^{e}=\left\{h \in \hat{H}_{m}^{+\infty} \hat{H}_{m} ; h \equiv 0 \text { em } \Omega\right\}
\end{gathered}
$$

definamos

$$
\not:=\mathscr{H}^{i} \dot{U} \psi^{e}
$$

onde $\dot{U}:=$ união disjunta

Note que por construção, toda $g \in \mathcal{i}^{i}$ é analitica em $\Omega$ e toda $h \in \mathcal{L}^{e}$ é analitica em $\bar{\Omega}^{c}$.

Considere o conjunto enumerável

$$
\left\{\hat{\mathrm{H}}_{j} ; \hat{\mathrm{H}}_{j}=\hat{\mathrm{H}}_{n} \text { se } \mathrm{j} \text { é impar e } \overline{\mathrm{H}}_{j}=\overline{\mathrm{H}}_{\mathrm{m}} \text { se } j \text { é par }\right\}_{j=1}^{+\infty}
$$


Vamos munir o espaço $\$$ de um produto interno tal que ele seja completo em relação à norma definida por esse produto interno.

Defina uma função $\langle$,$\rangle : \forall \times \rightarrow \mathbb{C}$ de modo análogo ao produto interno no espaco. $\mathrm{H}_{j}, i . \bar{e}$, por

$$
\langle f, g\rangle_{\not}=\left(f, \tilde{\mathbb{K}}_{\mathscr{g}} g\right) \quad f, g \in \not
$$

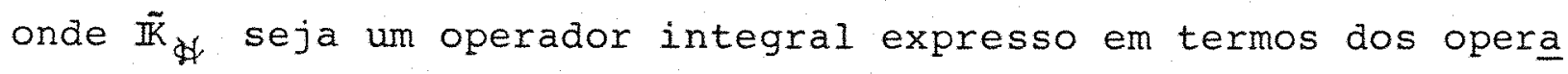
dores integrais $\tilde{\mathbb{K}}_{j}$ que definem o produto interno em cada espaço $\hat{\mathrm{H}}_{j}$. Desde que as propriedades do operador $\tilde{\mathbb{K}}_{j}$ depende das propreidades de seu núcleo $\tilde{K}_{j}$, é natural definir o operador $\tilde{\mathbb{K}}_{4}$ por

$$
\tilde{\mathbb{K}}_{\psi}(f(\zeta))=\int_{\Gamma} \tilde{K}_{\psi}\left(\zeta, \zeta^{\prime}\right) f\left(\zeta^{\prime}\right) d s^{\prime}, f \in d
$$

onde o nücleo $\tilde{K}_{\oiint}$ é da forma

$$
\tilde{K}_{q}\left(\zeta, \zeta^{\prime}\right)=\sum_{j=1}^{+\infty} c_{j} \tilde{K}_{j}\left(\zeta, \zeta^{\prime}\right),\left(\zeta, \zeta^{\prime}\right) \in \Gamma \times \Gamma
$$

com coeficientes $c_{j}$ apropriados tal que a série (III.6.6) convirja uniforme e absolutamente em $\Gamma \times \Gamma$. Portanto, deve-se achar um majorante para o módulo dos núcleos $\tilde{\mathrm{K}}_{\mathrm{j}}$.

$$
\text { Se jé impar, } \hat{\mathrm{H}}_{j}=\hat{\mathrm{H}}_{n} \in \mathcal{H}^{\dot{i}} \text { e o núcleo } \tilde{\mathrm{K}}_{j} \text { está dado }
$$

por 


$$
\tilde{K}_{j}\left(\zeta, \zeta^{\prime}\right)=\frac{r_{j}}{4 \pi^{2}} \oint_{C\left(z_{j} ; r_{j}\right)} \frac{e^{i\left(\theta\left(\zeta^{\prime}\right)-\theta(\zeta)\right)}}{(\bar{\zeta}-\bar{z})\left(\zeta^{\prime}-z\right)} d s
$$

isto, pela definição de $\Downarrow^{i}$. Assim,

$$
\left|\tilde{K}_{j}\left(\zeta, \zeta^{\prime}\right)\right| \leqq \frac{r_{j}}{4 \pi^{2}} \oint_{C\left(z_{j} ; r_{j}\right)} \frac{d s}{|\bar{\zeta}-\bar{z}| \mid \zeta^{\prime-z \mid}}
$$

como $\zeta, \zeta^{\prime} \in \Gamma$ e $z \in C\left(z_{j} ; r_{j}\right)$ onde $0<r_{j}<1$ então $a_{j} \leqq|\bar{\zeta}-\bar{z}|$ e $a_{j} \leqq\left|\zeta^{\prime}-z\right|$, onde $\alpha_{j}=\operatorname{aist}\left(c\left(z_{j} ; r_{j}\right), \Gamma\right)>0$.

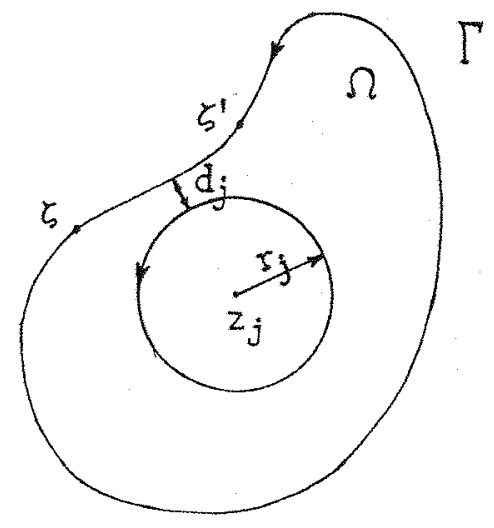

$$
\text { z-plano }
$$

Portanto

$$
\left|\tilde{k}_{j}\left(\zeta, \zeta^{\prime}\right)\right| \leqq \frac{r_{j}}{4 \pi^{2} a_{j}^{2}} \oint_{c\left(z_{j} ; r_{j}\right)} d s=\frac{r_{j}^{2}}{2 \pi a_{j}^{2}}<\frac{1}{a_{j}^{2}}
$$

Então

$$
\text { (III.6.7) } \quad\left|\tilde{K}_{j}\left(\zeta, \zeta^{\prime}\right)\right|<\frac{1}{d_{j}^{2}} \text { para todo j impar. }
$$

Agora, se jé par, tem-se $\hat{\mathrm{H}}_{j}=\mathrm{H}_{\mathrm{m}} \in \mathcal{H}^{\mathrm{e}}$. Desde que o espaço $\hat{\mathrm{H}}\left(\Gamma^{\prime}, z_{0}^{1}, r_{z_{0}^{\prime}}<, z_{0}^{\prime}\right)$ e sua imagem $\hat{\mathrm{H}}\left(\Gamma, I / z_{0}^{1}, r_{z_{0}^{\prime}},<, \gamma_{1 / z_{0}^{\prime}}\right)$ pela 
transformação conforme $z^{\prime} \longrightarrow \frac{1}{z^{\prime}}$ possuem o mesmo produto interno, basta analisar o núcleo $\tilde{\mathrm{K}}_{j}$ para o produto interno do espaço $\hat{\mathrm{H}}\left(\Gamma^{\prime}, z_{0}^{\prime}, r_{z_{0}^{\prime}},<_{z_{0}^{\prime}}\right)$, isto, pela definição de $\mathcal{H}^{e}$.

Analogamente ao caso em que j é impar, a expressão do núcleo $\tilde{\mathrm{k}}_{j}$ está dado por

$$
k_{j}\left(\frac{1}{\zeta} \frac{1}{\zeta^{\prime}}\right)=\frac{r_{j}^{\prime}}{4 \pi^{2}} \oint_{C\left(z_{j}^{\prime} ; r_{j}^{\prime}\right)} \frac{e^{i\left(\theta\left(1 / \zeta^{\prime}\right)-\theta(1 / \zeta)\right)}}{\left(\frac{1}{\bar{\zeta}}-\bar{z}^{\prime}\right)\left(\frac{1}{\zeta^{\prime}}-z^{\prime}\right)} d s .
$$

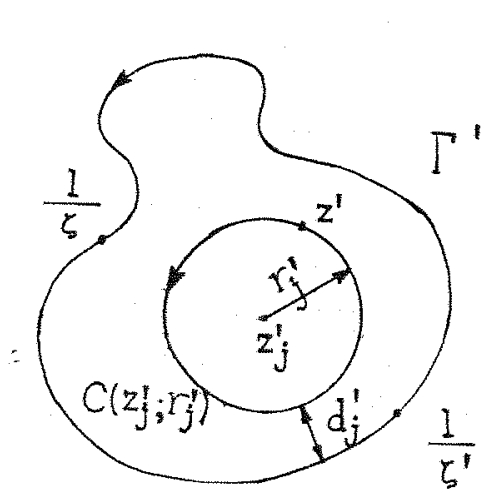

$z^{*}-$ plano

Então

$$
\left|\tilde{K}_{j}\left(\frac{1}{\zeta}, \frac{1}{\zeta^{\prime}}\right)\right| \leqq \frac{r_{j}^{\prime}}{4 \pi^{2}} \oint_{C\left(z_{j}^{\prime} ; r_{j}^{\prime}\right)} \frac{d s}{\left|\frac{1}{\zeta}-\bar{z}^{\prime}\right|\left|\frac{1}{\zeta^{\prime}}-\bar{z}^{\prime}\right|}
$$

Como $1 / \zeta, 1 / \zeta^{\prime} \in \Gamma^{\prime}$ e $z^{\prime} \in C\left(z_{j}^{\prime} ; r_{j}^{\prime}\right)$ onde $0<r_{j}^{\prime}<1$, temos que $\quad d_{j}^{\prime} \leqq\left|\frac{1}{\zeta}-\bar{z}^{\prime}\right|$ e $d_{j}^{\prime} \leqq\left|\frac{1}{\zeta^{\prime}}-z^{\prime}\right|$. Portanto

$$
\left|\tilde{K}_{j}\left(\frac{1}{\zeta}, \frac{1}{\zeta^{\prime}}\right)\right| \frac{r_{j}^{\prime}}{4 \pi^{2} a_{j}^{\prime 2}} \phi_{c\left(z_{j}^{\prime} ; r_{j}^{\prime}\right)} d s=\frac{r_{j}^{2}}{2 \pi d_{j}^{\prime 2}}<\frac{1}{d_{j}^{2}}
$$

Então 
(III.6.8)

$$
\begin{gathered}
\left|\tilde{\mathrm{K}}_{j}\left(\frac{1}{\zeta}, \frac{1}{\zeta^{\prime}}\right)\right|<\frac{1}{a_{j}^{j^{2}}} \text { para todo j par, e todo } \\
\left(\zeta, \zeta^{\prime}\right) \in \Gamma \times \Gamma .
\end{gathered}
$$

Tomando $\delta_{j}=\min \left\{d_{j}, d_{j}^{\xi}\right\}$, de $(I I I .6 .7)$ e $(I I I .6 .8)$, segue-se

$$
\left|\tilde{K}_{j}\left(\zeta, \zeta^{\prime}\right)\right|<\frac{1}{\delta_{j}^{2}} \text { para todo } j \in \mathbb{Z}^{+} \text {. }
$$

Portanto, basta toma $c_{j}=\frac{\delta_{j}^{2}}{2}$ para que a série em (III.6.6) convirja uniforme e absolutamente em $\Gamma \times \Gamma$. De fato,

$$
\left|\sum_{j=1}^{+\infty} \frac{\delta_{j}^{2}}{2^{j}} k_{j}\left(\zeta, \zeta^{\prime}\right)\right| \leqq \sum_{j=1}^{+\infty} \frac{\delta_{j}^{2}}{2^{j}}\left|\tilde{k}_{j}\left(\zeta, \zeta^{\prime}\right)\right|<\sum_{j=1}^{\infty} \frac{1}{2^{j}}<+\infty .
$$

Em consequência, a função $<,>$ : $\$ \times \$$ definido por

$$
\langle f, g\rangle \notin=\int_{\Gamma} f(\zeta)[\tilde{\mathbb{K}} \Psi(g(\zeta))] d s
$$

onde o nücleo $\tilde{K}_{\oiint}$ do operador integral $\tilde{\mathbb{K}}_{\mathscr{\Psi}}$ é da forma

$$
\tilde{\mathrm{K}}_{\psi}\left(\zeta, \zeta^{\prime}\right)=\sum_{j=1}^{+\infty} \frac{\delta_{j}^{2}}{2^{j}} k_{j}\left(\zeta, \zeta^{\prime}\right) \text { para todo }
$$

$$
\left(\zeta, \zeta^{\prime}\right) \in \Gamma \times \Gamma
$$

é um produto interno para $\Phi$.

Logo, a norma que provém deste produto interno,

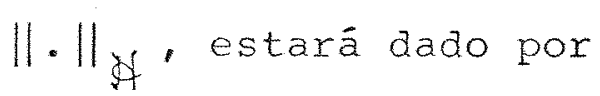


(III.6.10) $\quad\|f\|_{j}^{2}=\sum_{j=1}^{+\infty} \frac{\delta}{2^{j}}\|f\|_{\hat{H}_{j}}^{2} \quad$ para toda $f \in$

onde

$$
\|\mathrm{f}\|_{\hat{\mathrm{H}}_{j}}^{2}=2 \pi \sum_{\mathrm{m}=-}^{+\infty} r_{j}^{2|m+1|}\left|\mathrm{a}_{j, \mathrm{~m}}\right|^{2}
$$

Finalmente, provaremos que o espaço, $\left(\Psi_{1}\|\cdot\|_{\not f}\right)$ é um espaço de Hilbert.

Seja $\left\{f_{n}\right\}$ uma sequēncia de cauchy em $\left(\sharp,\|\cdot\|_{\not \psi}\right)$, de (III.6.10) temos que

$$
\left\|f_{p}-f_{q}\right\| \longrightarrow 0 \text { quando } p, q \longrightarrow+\infty \text { implica }\left\|f_{p}-f_{q}\right\|_{\hat{H}_{j}} \longrightarrow 0
$$

quando $\mathrm{p}, \mathrm{q} \rightarrow+\infty$

$$
\begin{gathered}
\text { No espaco } \hat{\mathrm{H}}_{j} \text {, se j é impar, temos: } \\
\left\|f_{p}-f_{q}\right\|_{\hat{H}_{j}}=\left\|w_{f_{p}}^{i}-w_{f_{q}}^{i}\right\|_{\hat{\mathrm{H}}_{j}}
\end{gathered}
$$

Segue da definição dos espaços $\hat{\mathrm{H}}_{j} \in \mathcal{H}^{i}$. E como $\hat{\mathrm{H}}_{j}$ é um espaco completo existe $g_{j} \in \hat{\mathrm{H}}_{j} ; g_{j} \equiv 0$ em $\bar{\Omega}^{\mathrm{C}}$ e $\left\|w_{\hat{f}_{p}}^{i}-g_{j}\right\|_{\hat{\mathrm{H}}_{j}} \rightarrow 0$ quando $\mathrm{p} \longrightarrow+\infty$.

Se jé par, da definição dos espaços $\hat{\mathrm{H}}_{j}$ e $\mathcal{H}^{e}$, segue

$$
\left\|f_{p}-f_{q}\right\|_{\hat{H}_{j}}=\left\|w_{f_{p}}^{e}-w_{f_{q}}^{e}\right\|_{\hat{H}_{j}}
$$

Pelo mesmo racicinio que anteriormente, existe $h_{j} \in \hat{H}_{j}$ i $h_{j} \equiv 0$ em $\Omega$ e $\left\|w_{f_{p}}^{e}-h_{j}\right\|_{\hat{H}_{j}} \rightarrow 0$ quando $p \rightarrow+\infty$. 
Defina $g:=g_{j}$ em $\overline{\mathrm{H}}_{j}, j$ impar e $\mathrm{h}:=\mathrm{h}_{j}$ em $\overline{\mathrm{H}}_{j}$, j par. Vemos que $g \in \psi^{i}$ e $h \in \mathcal{H}^{e}$.

Tomando $f=g+h . \quad$ Segue-se $\left\|f_{p}-f\right\|_{j} \longrightarrow 0$ quando $\mathrm{p} \longrightarrow+\infty$. Logo $\left(\not,\|.\|_{1}\right)$ é um espaço de Hilbert.

000 


\section{CAPETULO IV}

\section{APLICACAO A FORMA DIFERENCIAI $\bar{z} d z$}

Nosso objetivo neste capitulo é utilizar alguns dos resultados do Capítulo III para determinar quando a integral (IV. 1)

$$
\int_{\Gamma} h(\zeta) \bar{\zeta} d \zeta
$$

é diferente de zero, onde h è uma função positiva sobre a curva fechada simples $\Gamma$. Veremos que isto vai depender da geometria da curva $\Gamma$.

Em primeiro lugar, vejamos que relação existe entre a integral dada em (IV.1) e o produto interno definido no Capi tulo III.

Considere a função 1 , de (III.3.2) segue

$=\frac{1}{\rho^{2}} \zeta(s)$ onde $\rho=\rho(s)>0$, portanto

$$
(\tilde{\mathbb{K}} 1)(\zeta(s))=\operatorname{ir}^{2} \bar{\zeta}(s)^{-1} \mathrm{e}^{-i \theta(\zeta(s))}
$$

Seja $\zeta(s)=p e^{i \theta(\zeta(s))}$, então $\bar{\zeta}^{-1}(s)=\frac{1}{\rho} e^{i \theta(\zeta(s))}=$

$$
(\tilde{\mathbb{K}} 1)(\zeta(s))=i\left(\frac{r}{\rho}\right)^{2} \zeta(s) e^{-i \theta(\zeta(s))}
$$

Seja h uma função positiva então $\rho^{2} \mathrm{~h}$ é uma funcão po sitiva e temos

$$
\left\langle\rho^{2} h, 1\right\rangle=\int_{\Gamma} \rho^{2} h(\zeta(s))(\tilde{\mathbb{K}} I)(\zeta(s)) d s
$$


Substituindo (IV.1) no segundo membro da igualdade anterior, obtemos

$$
\begin{aligned}
\left\langle\rho^{2} h, 1\right\rangle & =i r^{2} \int_{\Gamma} \bar{h}(\zeta) \zeta d \bar{\zeta} \\
& =-i r^{2} \int_{\Gamma} h(\zeta) \bar{\zeta} d \zeta
\end{aligned}
$$

Então

(IV. 3)

$$
\int_{\Gamma} h(\zeta) \bar{\zeta} d \zeta=\frac{i}{r^{2}}\left\langle\rho^{2} h, 1\right\rangle
$$

de onde

(IV. 4)

$$
\operatorname{Im}\left(\int_{\Gamma} h(\zeta) \bar{\zeta} d \zeta\right)=\operatorname{Re}\left(\frac{1}{r^{2}}\left\langle\rho^{2} h, 1>\right)\right.
$$

Por outro lado, sabemos que

$$
(\tilde{\mathbb{K}} I)(\zeta(s))=\int_{\Gamma} \tilde{K}\left(\zeta, \zeta^{\prime}\right) d s^{\prime}
$$

onde

$\hat{K}\left(\zeta, \zeta^{\prime}\right)=\frac{1}{2 \pi}\left(\frac{r^{2}}{\bar{\zeta} \zeta^{\prime}-r^{2}}-\frac{r^{-2}}{\bar{\zeta} \zeta^{\prime}-r^{-2}}\right) e^{i\left(\theta^{\prime}-\theta\right)}$ para todo $\left(\zeta, \zeta^{\prime}\right) \in \Gamma \times \Gamma$.

Escreva $\tilde{\mathrm{K}}\left(\zeta, \zeta^{\prime}\right)$ da seguinte forma

$$
K\left(\zeta, \zeta^{\prime}\right)=K_{I}\left(\zeta, \zeta^{\prime}\right)+K_{E}\left(\zeta, \zeta^{\prime}\right) \quad\left(\zeta, \zeta^{\prime}\right) \in \Gamma \times \Gamma
$$

onde 


$$
\begin{aligned}
& \tilde{\mathrm{K}}_{I}\left(\zeta, \zeta^{\prime}\right)=\frac{1}{2 \pi} \cdot \frac{r^{2}}{\zeta \zeta^{\prime}-r^{2}} e^{i\left(\theta^{\prime}-\theta\right)}, \quad e \\
& \tilde{\mathrm{K}}_{E}\left(\zeta, \zeta^{\prime}\right)=-\frac{1}{2 \pi} \cdot \frac{r^{-2}}{\bar{\zeta} \zeta^{\prime}-r^{-2}} e^{i\left(\theta^{\prime}-\theta\right)} .
\end{aligned}
$$

Como a função $1 \in \mathbb{A}(\Omega)$, onde $\Omega$ é a região limitada por $\Gamma$, tem-se

$$
(\tilde{\mathbb{K}} 1)(\zeta(s))=\int_{\Gamma} \tilde{K}_{I}\left(\zeta, \zeta^{\prime}\right) d s^{\prime} .
$$

Assim, estamos interessados em saber quando Re $\left(\tilde{K}_{I}\right)$, a parte real de $\tilde{K}_{I}$ possui sinal constante, seja $\bar{\zeta}=\rho e^{-i \gamma}$ e $\zeta^{\prime}=\rho^{\prime} e^{i \gamma^{\prime}}$

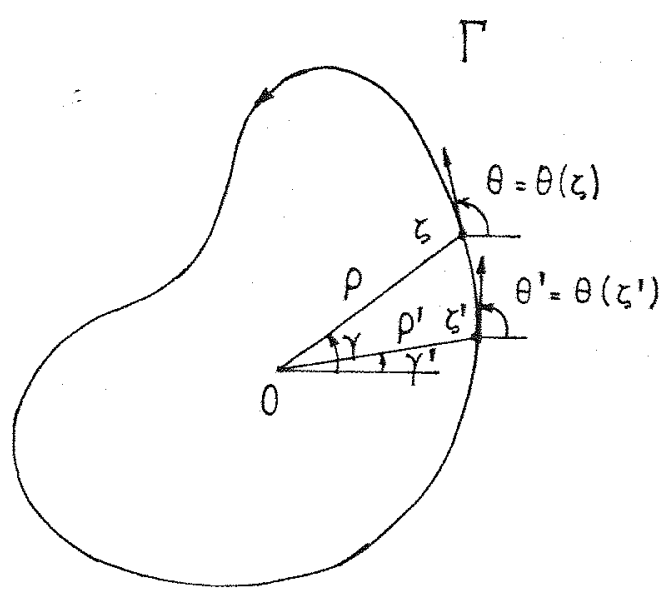


então

$$
\begin{aligned}
\tilde{\mathrm{K}}_{I}\left(\zeta, \zeta^{\prime}\right)= & \frac{r^{2}}{2 \pi} \cdot \frac{e^{i\left(\theta^{\prime}-\theta\right)}}{\rho \rho^{\prime} e^{-i\left(\gamma-\gamma^{\prime}\right)}-r^{2}} \\
= & \frac{r^{2}}{2 \pi}\left(\frac{\cos \left(\theta^{\prime}-\theta\right)+i \operatorname{sen}\left(\theta^{\prime}-\theta\right)}{\left(\rho \rho^{\prime} \cos \left(\gamma^{\prime} \gamma^{\prime}\right)-r^{2}\right)-i \rho \rho^{\prime} \operatorname{sen}\left(\gamma-\gamma^{\prime}\right)}\right) \\
= & \frac{r^{2}}{2 \pi}\left(\frac{\left(\rho \rho^{\prime} \cos \left((\gamma-\theta)-\left(\gamma^{\prime}-\theta^{\prime}\right)\right)-r^{2} \cos \left(\theta^{\prime}-\theta\right)\right)}{\left(\rho \rho^{\prime}\right)^{2}-2 \rho \rho^{\prime} r^{2} \cos \left(\gamma-\gamma^{\prime}\right)+r^{4}}+\right. \\
& \left.+\frac{\left.i\left(\rho \rho^{\prime} \operatorname{sen}^{2}(\gamma-\theta)-\left(\gamma^{\prime}-\theta^{\prime}\right)\right)-r^{2} \operatorname{sen}\left(\theta^{\prime}-\theta\right)\right)}{\left(\rho \rho^{\prime}\right)^{2}-2 \rho \rho^{\prime} r^{2} \cos \left(\gamma-\gamma^{\prime}\right)+r^{4}}\right) .
\end{aligned}
$$

Portanto, a parte real de $\tilde{\mathrm{K}}_{I}\left(\zeta, \zeta^{\prime}\right)$ está dada por $\operatorname{Re}\left(\tilde{R}_{I}\left(\zeta, \zeta^{\prime}\right)\right)=\frac{r^{2}}{2 \pi}\left[\frac{\rho \rho^{\prime} \cos \left((\gamma-\theta)-\left(\gamma^{\prime}-\theta^{\prime}\right)\right)-r^{2} \cos \left(\theta^{\prime}-\theta\right)}{\left(\rho \rho^{\prime}\right)^{2}-2 \rho \rho^{\prime} r^{2} \cos \left(\gamma-\gamma^{\prime}\right)+r^{4}}\right]$.

Se

$$
\begin{aligned}
& \rho_{0}=\min \left\{\rho, \rho^{\prime}\right\}, \text { temos } \\
& \rho \rho^{\prime} \cos \left((\gamma-\theta)-\left(\gamma^{\prime}-\theta^{\prime}\right)\right)-r^{2} \cos \left(\theta^{\prime}-\theta\right) \geqq \\
& \geqq \rho_{0}^{2} \cos \left((\gamma-\theta)-\left(\gamma^{\prime}-\theta^{\prime}\right)\right)-r^{2}
\end{aligned}
$$

Suponha que $\Gamma$ é uma curva que satisfaz

$$
\cos \left((\gamma-\theta)-\left(\gamma^{\prime}-\theta^{\prime}\right)\right)>\frac{x^{2}}{\rho_{0}^{2}}
$$

tomando $\delta_{0}=\operatorname{arc} \cos \left(\frac{r^{2}}{\rho_{0}^{2}}\right)$, segue $0<\delta_{0}<\frac{\pi}{2}$ de onde 
(IV. 5)

$$
\left|(\gamma-\theta)-\left(\gamma^{\prime}-\theta^{\prime}\right)\right|<\delta_{0}
$$

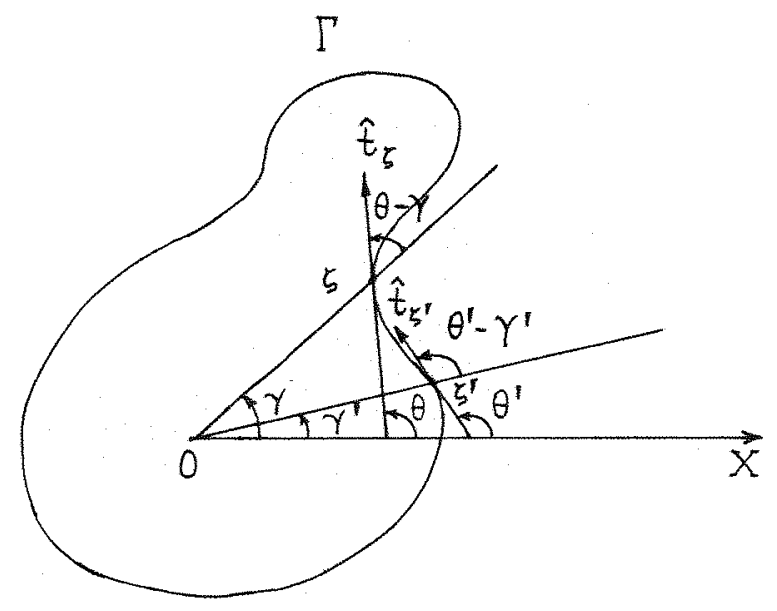

Note que, dado um āngulo fixo $\Delta_{0}$, uma curva $\Gamma^{\prime}$ que satisfaz

(IV. 6)

$$
=\left|(\gamma-\theta)-\Delta_{0}\right|<\frac{\delta_{0}}{2}
$$

tambëm satisfaz (IV.5). Segue-se que o conjunto de curvas $\Gamma^{\prime}$ que satisfazem (IV.6) definem uma vizinhança $V(T)$ de classe $\mathrm{C}^{1}$ de $\Gamma$ onde $\operatorname{Re}\left(\tilde{\mathrm{K}}_{I}\right)$ possui sinal constante.

$$
\text { No caso } \Gamma=s^{1} \text {, temos } \delta_{0}=0 \mathrm{e} \Delta_{0}=\frac{\pi}{2} \text {. }
$$

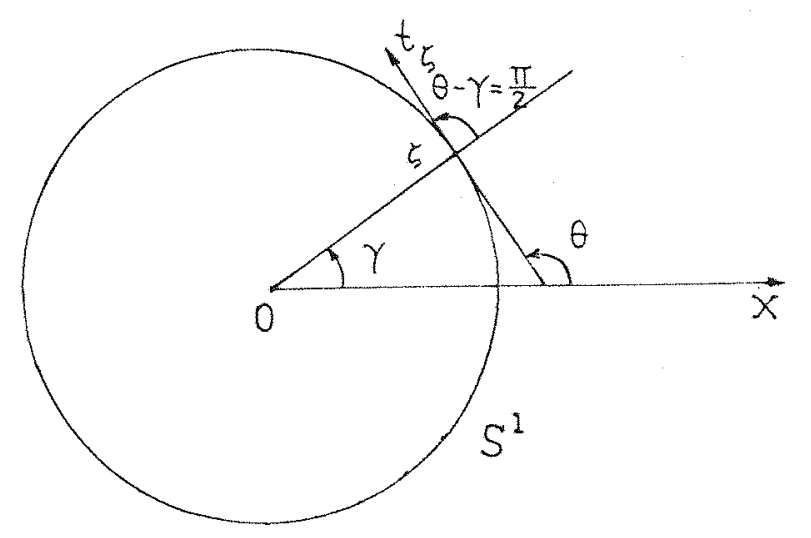


De (IV.4), segue-se que

$$
\operatorname{Im} \int_{\Gamma} \bar{h}(\zeta) \bar{\zeta} d \zeta
$$

possui sinal constante, se a curva $\Gamma$ satisfaz (IV.5) e portanto para toda curva $\Gamma^{\prime}$ na vizinhança. $V(\Gamma)$.

000 


\section{BIBEIOGRAEIA}

[1] Hille, E. - Analytic Functions Theory, Vol. II. Gin and Company, Boston, 1962.

[2] Hönig, Ch.S. Análise Funcional e o Problema de Sturn-Liouville. Instituto de Matemática Pura e Aplicada, 2a Edição, Rio de Janeiro, 1971.

[3] Mikhlin, S.G. . Integral Equations and Their Appplications to Certain Problems in Mechanics, Mathematical Physics and Technology. Pergamon Press, London, 1957.

[4] Monakov, V.N. Boundary - Value Problems with Free Boundaries for Elliptics Systems of Equations. Translations of Mathematical Monographs, 57. Providence - R.I. AMS, 1983.

[5] Mukherjea, A. and Pothoven, K. . Real and Funcional Analysis. Plemun Press, New York, 1978.

[6] Rudin, W. . Real and Complex Analysis, Second Edition, McGraw-Hill, New York, 1983. 